\title{
Higher Radix Floating-Point Representations for FPGA-Based Arithmetic
}

Bryan Christopher Catanzaro

Brigham Young University - Provo

Follow this and additional works at: https://scholarsarchive.byu.edu/etd

Part of the Electrical and Computer Engineering Commons

\section{BYU ScholarsArchive Citation}

Catanzaro, Bryan Christopher, "Higher Radix Floating-Point Representations for FPGA-Based Arithmetic" (2005). Theses and Dissertations. 311.

https://scholarsarchive.byu.edu/etd/311

This Thesis is brought to you for free and open access by BYU ScholarsArchive. It has been accepted for inclusion in Theses and Dissertations by an authorized administrator of BYU ScholarsArchive. For more information, please contact scholarsarchive@byu.edu, ellen_amatangelo@byu.edu. 
HIGHER RADIX FLOATING-POINT REPRESENTATIONS FOR FPGA-BASED ARITHMETIC

Bryan C. Catanzaro

A thesis submitted to the faculty of Brigham Young University in partial fulfillment of the requirements for the degree of

Master of Science

Department of Electrical and Computer Engineering

Brigham Young University

August 2005 

Copyright (c) 2005 Bryan C. Catanzaro

All Rights Reserved 



\title{
BRIGHAM YOUNG UNIVERSITY
}

\section{GRADUATE COMMITTEE APPROVAL}

\author{
of a thesis submitted by \\ Bryan C. Catanzaro
}

This thesis has been read by each member of the following graduate committee and by majority vote has been found to be satisfactory.

Date

Date

Date
Brent E. Nelson, Chair

Michael J. Wirthlin

Doran K. Wilde 



\section{BRIGHAM YOUNG UNIVERSITY}

As chair of the candidate's graduate committee, I have read the thesis of Bryan C. Catanzaro in its final form and have found that (1) its format, citations, and bibliographical style are consistent and acceptable and fulfill university and department style requirements; (2) its illustrative materials including figures, tables, and charts are in place; and (3) the final manuscript is satisfactory to the graduate committee and is ready for submission to the university library.

Date

Accepted for the Department
Brent E. Nelson

Chair, Graduate Committee
Michael A. Jensen

Graduate Coordinator

Accepted for the College

Douglas M. Chabries

Dean, Ira A. Fulton College

of Engineering and Technology 



\author{
ABSTRACT \\ HIGHER RADIX FLOATING-POINT REPRESENTATIONS FOR \\ FPGA-BASED ARITHMETIC \\ Bryan C. Catanzaro \\ Department of Electrical and Computer Engineering \\ Master of Science
}

Field Programmable Gate Arrays (FPGAs) are increasingly being used for high-throughput floating-point computation. It is forecasted that by 2009, FPGAs will provide an order of magnitude greater sustained floating-point throughput than conventional processors [1]. FPGA implementations of floating-point operators have historically been designed to use binary floating-point representations, as do general purpose processors. Binary representations were chosen as the standard over three decades ago because they provide maximal numerical accuracy per bit of floatingpoint data. However, the unique nature of FPGA-based computation makes numerical accuracy per unit of FPGA resources a more important measure of the usefulness of a given floating-point representation.

From this viewpoint, higher radix floating-point representations are well suited to FPGA-based computations, especially high precision calculations which require the support of denormalized numbers. This work shows that higher radix representations lead to more efficient use of FPGA resources. For example, a hexadecimal floatingpoint adder provides a 30\% lower Area-Time product than its binary counterpart, 

and a hexadecimal floating-point multiplier has a $13 \%$ lower Area-Time product than its binary counterpart. This savings occurs while still delivering equal worst-case and better average-case numerical accuracy. This work presents a family of higher radix floating-point representations that are designed specifically to interoperate with standard IEEE floating-point, allowing the creation of floating-point datapaths which operate on standard binary floating-point data, yet use higher radix representations internally. Such datapaths provide higher performance by any measure: they are more accurate numerically, consume less FPGA resources and have shorter latencies. When taking into consideration the unique nature of FPGA-based computing systems, this work shows that binary floating-point representations are not optimal for most FPGA-based arithmetic computations. Higher radix representations can therefore be a useful tool for building efficient custom floating-point datapaths on FPGAs. 



\section{Contents}

List of Tables

List of Figures $\quad$ Xviii

1 Introduction 1

2 Background 5

2.1 Mathematical Terminology ................ 5

2.2 Floating-Point Format Background . . . . . . . . . . . . 8

2.2.1 IEEE Format Details . . . . . . . . . . . . . 8

2.2 .2 Rounding ..................... 10

2.2.3 Treatment of Special Numbers . . . . . . . . . . . 11

2.2.4 Quadruple Precision . . . . . . . . . . . . . 12

2.3 Historical Background . . . . . . . . . . . . . . . 14

2.4 On the Need for Bit-Identical Results . . . . . . . . . . . . . . . 17

2.5 Related Work . . . . . . . . . . . . . . . . 18

2.5.1 Floating-Point Arithmetic on FPGAs . . . . . . . . . . . . . 18

2.5.2 Higher Radix Floating-Point Implementations . . . . . . . . . 20

3 Proposed Representation 23

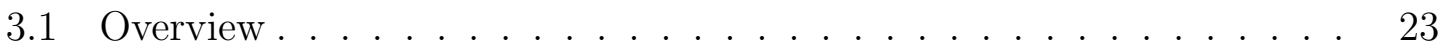

3.2 Radix Point Position . . . . . . . . . . . . . . . 23

3.3 Encoding . . . . . . . . . . . . . . . . . . . . . . . 28

3.4 Flag Bits. . . . . . . . . . . . . . . . . . . . 31

3.5 Dynamic Range . . . . . . . . . . . . . . . . . . . 32 
3.6 Numerical Accuracy . . . . . . . . . . . . . . . . . . . . . 35

3.6.1 Worst Case Relative Error . . . . . . . . . . . . 35

3.6.2 Relative Significance Space Density . . . . . . . . . . . 37

3.6 .3 Gap Functions . . . . . . . . . . . . . . . . 40

3.7 Rounding Procedures . . . . . . . . . . . . . . . . . . . . . . . 44

3.8 Summary $\ldots \ldots \ldots \ldots \ldots \ldots$

4 Implementation 47

4.1 Unpipelined Adder . . . . . . . . . . . . . . . . . . . . . 48

4.2 Unpipelined Multiplication . . . . . . . . . . . . . . 52

4.3 Converter Hardware . . . . . . . . . . . . . . . 56

4.4 Pipelined Operators . . . . . . . . . . . . . . . 57

4.5 Floating-Point Unit Building Blocks . . . . . . . . . . . . . . 60 60

4.5.1 Priority Encoder . . . . . . . . . . . . . . . 60

4.5.2 Normalizing and Aligning Shifters . . . . . . . . . . 60 60

4.6 Future Work . . . . . . . . . . . . . . . . . . . 64

5 Conclusions 65

A Reducing Embedded Multiplier Usage 69

A.1 Justification . . . . . . . . . . . . . . . . . . 69 69

A.2 Factorization ................ 71

A.3 Architecture . . . . . . . . . . . . . 72

A.4 Implementation . . . . . . . . . . . . . 78

Bibliography 81 


\section{List of Tables}

2.1 Round Logic . . . . . . . . . . . . . . . . . . . . 11

2.2 Special Numbers . . . . . . . . . . . . . . . . 11

2.3 Addition Special Cases . . . . . . . . . . . . . . . 13

2.4 Subtraction Special Cases . . . . . . . . . . . . . . . . . 13

2.5 Multiplication Special Cases . . . . . . . . . . . . . 13

2.6 Division Special Cases . . . . . . . . . . . . . . . 13

3.1 Encoded Numbers in Different Representations . . . . . . . . . . 29

3.2 Floating-point Word Size with Equalized Numeric Performance . . . . 30

3.3 Standard Special Case Logic . . . . . . . . . . . . . . . . . 31

3.4 Encoded Flags . . . . . . . . . . . . . . . . . . . . 32

3.5 Format Parameters . . . . . . . . . . . . . . . 34

3.6 Dynamic Range . . . . . . . . . . . . . . . . . . 34

4.1 Unpipelined Adder Area Comparison . . . . . . . . . . . . . . . . 5 51

4.2 Unpipelined Adder Timing Comparison . . . . . . . . . . . . . . 51

4.3 Unpipelined Multiplier Area Comparison . . . . . . . . . . . . . 55

4.4 Multiplier Timing Comparison . . . . . . . . . . . . 55

4.5 Converter Circuitry Area . . . . . . . . . . . . . . 57

A.1 Multiplier Sizes . . . . . . . . . . . . . . . 78 


\section{List of Figures}

2.1 Standard Floating-Point Formats $\ldots \ldots \ldots \ldots$

3.1 Externally Radix 2, Internally Radix 16 System . . . . . . . . . 24

3.2 Exponent Mapping, $\delta^{\prime}=\frac{1}{\nu} \ldots \ldots \ldots \ldots \ldots \ldots \ldots \ldots$

3.3 Exponent Mapping, $\delta^{\prime}=0 \ldots \ldots \ldots \ldots \ldots \ldots$

3.4 Floating-Point Format Size Increase versus Radix . . . . . . . . . 30

3.5 Relative Worst Case Accuracy versus FP Word Size . . . . . . . . . . 36

3.6 Density Comparison _ . . . . . . . . . . . . . . . . . . . . 39

3.7 Relative Significance Space Density versus FP Word Size . . . . . . 41

3.8 Gap functions for 32-bit Fixed-Point and 32-bit Floating-Point . . . 42

3.9 Gap Functions for Radix 2 and Radix 16 Representations . . . . . . 42

4.1 Floating-point Adder . . . . . . . . . . . . . . . . . . . . . 48

4.2 Area for Unpipelined Adders Normalized to Radix 2 Adder . . . . . 5 50

4.3 Latency for Unpipelined Adders Normalized to Radix 2 Adder . . . . 50

4.4 Floating-point Multiplier . . . . . . . . . . . . . . . 52

4.5 Area for Unpipelined Multipliers Normalized to Radix 2 Multiplier . . 54

4.6 Latency for Unpipelined Multipliers Normalized to Radix 2 Multiplier 54

4.7 Area of Pipelined Operators in Slices . . . . . . . . . . . . 58

4.8 Period of Pipelined Operators in Nanoseconds . . . . . . . . . 58

4.9 Pipelined Area Time Products Normalized to Radix 2 . . . . . . . . 59

4.10 Radix 2, 25 Bit Priority Encoder . . . . . . . . . . . 61

4.11 Radix 16, 31 Bit Priority Encoder . . . . . . . . . . . . . . 62

4.12 Radix 16 Normalizing Shifter _. . . . . . . . . . . 6 63

4.13 Radix 2 Normalizing Shifter . . . . . . . . . . . . . . 63

A.1 Number of 18-bit Multipliers/Number of Lookup Tables . . . . . . . 70 
A.2 Number of Block Multipliers Versus Input Digit Width . . . . . . 73

A.3 Number of Partial Product Bits . . . . . . . . . . . . . . 74

A.4 Legend for Partial Product Arrays . . . . . . . . . . . . . . . 75

A.5 Standard Partial Product Array for Single Precision Multiply . . . . 75

A.6 Factored Partial Product Array for Single Precision Multiply . . . . 75

A.7 Standard Partial Product Array for Double Precision Multiply . . . 76

A.8 Factored Partial Product Array for Double Precision Multiply . . . . 76

A.9 Standard Partial Product Array for Quadruple Precision Multiply . . 77

A.10 Factored Partial Product Array for Quadruple Precision Multiply . . 77

A.11 Normalized Embedded Multiplier Usage _ . . . . . . . . . . . 79

A.12 Normalized Multiplier Slice Usage . . . . . . . . . . . . . . . . 79 


\section{Chapter 1}

\section{Introduction}

Arithmetic has been central to computing since its inception. Indeed, the first general purpose computers, such as ENIAC and UNIVAC, were developed as automated calculators for solving complex mathematical problems [2]. Although the scope of computing has broadened to include myriads of other tasks, arithmetic is still a vital part of computing.

At present, there are several ways of implementing mathematical calculations. The most traditional way is to use a von Neumann style computer, targeting a general purpose microprocessor or one more specifically designed for arithmetic calculation, such as a Digital Signal Processor (DSP). This approach is widely used because implementing a given computation is reduced to writing software, which has very low development costs and is well understood. Additionally, changing the functionality of such a computer is done simply and easily by running a different software program.

Although the von Neumann computer is well understood, the flexibility it provides naturally incurs performance penalties compared with a dedicated hardware implementation of a given calculation. The rise of the integrated circuit allowed the creation of Application Specific Integrated Circuits (ASICs), which trade flexibility for performance. ASICs are the idiot savants of the computing world: they achieve unmatched performance on one predetermined computation, but they are useless on any other. ASICs also suffer from extremely high development costs, due to the exponentially rising cost of first silicon from modern fabrication facilities, as well as the exhaustive validation process through which ASIC designs must pass before being fabricated. 
Application Specific Instruction Processors (ASIPs) are a relatively new outgrowth of the traditional von Neumann computing model. ASIPs assemble an assortment of heterogeneous, domain specific computing cores in a System-on-Chip solution. Being domain specific, an ASIP gives up a degree of flexibility compared to a traditional processor, and efficiently utilizing the multiple heterogenous resources which are found on an ASIP is a significant programming challenge. Still, many feel that ASIP platforms will provide a good balance of flexibility, performance, and cost in the future.

Configurable computers provide yet another way to implement mathematical computations: instead of hardwiring the calculation as in an ASIC, or using an array of domain specific computing cores as in an ASIP, allow the user to implement custom logic on a generic, reconfigurable compute fabric. This approach provides high performance with moderate development cost and a measure of design flexibility.

Although many different configurable computers are under research, the most prevalent way of implementing a configurable computer involves using Field Programmable Gate Arrays (FPGAs). FPGAs are an outgrowth of Programmable Logic Devices (PLDs), which were originally invented as an easy way to implement relatively simple logic functions, such as the next state in a finite state machine, or glue logic interfacing various computing devices. The astonishingly rapid increase in semiconductor fabrication capacity observed by Moore's law [3] has allowed FPGAs to become more than a useful way to implement simple custom logic, for which they were originally created. FPGAs are now becoming general compute fabrics, suitable for diverse applications such as network processing, genetic pattern matching, image and video processing, and communications processing.

Recent increases in FPGA capacity and capability have led to broader use of FPGA-based, custom floating-point arithmetic datapaths. When configurable resources were scarce, arithmetic calculations had to be implemented using fixed-point arithmetic. However, fixed-point arithmetic is difficult to use because the dynamic range of the calculation must be limited and known a priori in order to avoid underflow and overflow issues, which is not possible for all applications. Additionally, 
fixed-point arithmetic has numerical performance issues: although its accuracy is good for large numbers, small numbers are represented poorly, since leading zero bits needed to indicate small numbers in fixed-point representations compete with the numerically significant bits which contribute to numerical accuracy.

The steady and rapid growth of FPGA resources has increased FPGA floating point throughput to match or beat conventional floating-point processors, and because FPGA-based calculations are able to take advantage of Moore's law more efficiently than traditional processors, FPGA floating-point throughput is growing at a faster rate. Indeed, it has been forecasted that FPGAs will enjoy an order of magnitude higher throughput on double precision floating-point arithmetic than conventional CPUs by the year 2009 [1].

The general computing world has settled on floating point representations which conform to IEEE standard 754 [4], and to a lesser extent, IEEE standard 854 [5]. These standards play a crucial role in ensuring numerical robustness and code compatibility among machines of vastly different architectures. However, the choice of floating-point representation has such a dominant impact on FPGA implementation costs that the standards are often bent, giving the designer freedom to choose a custom floating-point representation in order to spend FPGA resources as efficiently as possible. For example, work has been done to automatically determine custom floating-point bitwidths for each node of a computation [6], and others have demonstrated the suitability of very tiny floating-point representations with much less precision and range than IEEE single precision [7].

Choosing non-standard floating-point representations by manipulating bitwidths is natural for the FPGA community, since bitwidth has such an obvious, first-order effect on circuit implementation costs. Besides the non-standard bitwidths, FPGAbased floating-point units often save hardware cost by omitting support for denormalized numbers as well as some of the rounding modes specified by the IEEE standards.

Although the impact of non-standard bitwidth floating-point representations on FPGA implementation is well known, the effect of non-standard radix floatingpoint representations has not been examined. The word "radix" in the context of 
computer arithmetic has acquired several meanings, which can be confusing. In this work, "radix" to refers to the numerical base of the floating point representation, meaning that the mantissa is interpreted to be composed of digits of some base, such as 2 or 10. High radix floating-point representations are those with radix greater than two. This is not to be confused with high radix Booth encoding for multiplication or high radix division algorithms, as found in references to "high radix" floating-point operators such as [8] or [9].

This thesis shows that higher radix floating-point representations, especially hexadecimal floating-point, are uniquely suited for FPGA-based computation, particularly when denormalized numbers are supported. Choosing a higher radix floatingpoint representation can reduce adder area by $25 \%$ and multiplier area by $12 \%$, and while still providing equal worst-case and better average-case numerical accuracy than the standard binary representation. Higher radix representations are justified from from a numerical perspective as well as through implementation results (Xilinx Virtex-II) for arithmetic operators which operate on a higher radix representation. This work presents a family of higher radix formats, designed specifically to interface cleanly and simply with IEEE 754 representations. The savings gained from using high radix arithmetic operators can be used to fit designs on a cheaper FPGA, increase numerical precision substantially, or gain performance by increasing on-chip parallelism. Because they are more efficient by all measures, high radix representations should be considered by designers of FPGA-based floating-point datapaths. 


\section{Chapter 2}

\section{Background}

\subsection{Mathematical Terminology}

Floating-point arithmetic approximates a real number $x$ by choosing an element of a finite set of exactly representable real numbers $S$, called the significance space [10]. There are many different ways of modeling floating-point representations mathematically. The following model of floating-point number representations has been chosen to show the details which are most important to this work, without overwhelming the reader with extraneous miscellany.

Given a floating-point representation, or significance space $S_{\beta}^{u}$, the members of $S_{\beta}^{u}$ have the form

$$
s \beta^{e} \beta^{\delta-u} \sum_{i=0}^{u-1} d_{i} \beta^{i}
$$

where $s= \pm 1$ represents the sign, $\beta$ is the base, or radix, $u$ is the number of $\beta$ ary digits in the mantissa, $d_{u-1} \cdots d_{0}$ are the digits of the mantissa themselves, with $d_{u-1}$ being the most significant digit. The exponent value is $e$, and $\beta^{\delta-u}$ is a term that accounts for the placement of the implied radix point. With this notation, the radix point is placed $\delta \beta$-ary digits into the mantissa, from the most significant side. Equivalently, we can incorporate the $\beta^{\delta-u}$ term into the mantissa value, which leads to interpreting the mantissa to be in the range $\left[\beta^{\delta-1}, \beta^{\delta}\right)$. 
In this work, we restrict ourselves to radices of the form $\beta=2^{\nu}$, which ensures that each digit $d_{i}$ is efficiently representable in binary. Expanding (2.1) into binary, with $\beta=2^{\nu}$, elements of $S$ have the form

$$
s 2^{\nu e} 2^{\nu \delta-t} \sum_{j=0}^{t-1} b_{j} 2^{j}
$$

where each $\beta$-ary digit $d_{i}$ from (2.1) is expanded into its binary form $b_{\nu(i+1)-1} \cdots b_{\nu i}$, and $t=\nu u$ is the number of bits in the binary encoding of the mantissa $\left(d_{0} \cdots d_{u-1}\right)$. The term $2^{\nu \delta-t}$ accounts for the placement of the implied binary point.

The mantissa bitwidth $t$ is required to be an integer, but no such restriction is made on on $u$, allowing fractional digits of radix $\beta$. Similarly, $\nu \delta$ must be a integer, but fractional $\delta$ is allowed. With this representation, the radix point is placed $\nu \delta$ bits into the mantissa from the most significant side, which may fall in the middle of a $\beta$-ary digit. In other words, the radix point functions as a binary point regardless of radix, and can be positioned between any bit of the mantissa, not just at boundaries of radix $\beta$ digits.

It is worth noting that some of these parameters are specific to a given floatingpoint representation, whereas others encode a floating-point value. More concretely, $u$ or $t, \nu$ or $\beta$, and $\delta$ define characteristics of a floating-point representation which are necessarily the same for all data expressed in that representation. Although it is possible to convert data between floating-point representations, such conversions must be undertaken with care, since they introduce subtle numerical challenges. Frequent conversions between floating-point representations have been advocated for FPGA-based floating-point datapaths [6], in an attempt to attain the most numerical performance per unit of FPGA resources. Nothing prevents higher radix floating-point operators to be used in a similar fashion. However, the focus of this work is on providing numerical results which are provably better than those produced by standard IEEE representations. It follows that we do not allow $u, t, \delta, \beta$ or $\nu$ to vary during the calculation, although we do examine a very restricted set of conversions to be used at the input and output gateways of the datapath, where data may be required to enter and exit the FPGA in conventional, IEEE representation. 
If $d_{u-1} \neq 0$, meaning that the most significant digit of the mantissa is nonzero, or equivalently for our representations, if the leading one is found within the most significant $\nu=\log _{2} \beta$ bits, the number is considered normalized, which canonicalizes a floating-point format by prohibiting redundant realizations of the same number. In a floating-point format without normalization, a given real number can be represented in multiple ways. For example, if normalization is not required, $2.781828=0.2781828 * 10^{1}=27.81828 * 10^{-1}$, and so forth. If normalization is required, only one of the possible representations of a given number is allowed, for example, 2.781828, and all other equivalent representations are disallowed.

If the leading digit is zero, or equivalently, if the leading one is not found within the most significant $\nu$ bits, the number is considered denormalized. In this work and in the IEEE representation, denormalized numbers are only permitted when representing exceptionally small numbers which cannot be encoded in a normalized format. FPGA implementations often disallow denormalized numbers in general, forcing results to zero that should be represented in denormalized form. This lack of gradual underflow, while it saves hardware, can be very deleterious to numerical accuracy, and so support for denormalized numbers is required for some types of applications [1].

Normalization incurs a significant hardware cost, because it necessitates keeping track of the leading one and manipulating the mantissa so that the leading one is always positioned at the most significant end of the mantissa. Since the leading one may move during a calculation, a significant amount of hardware is required to accomplish this. Despite these costs, floating-point numbers are normalized because normalization preserves accuracy by keeping the mantissa bits significant. The canonicalization which it provides also enables easy comparison of two floating-point numbers, which is a crucial step in the add operation.

Since normalizing is expensive, it has been recently suggested [11] that using unnormalized floating-point formats can offer significant hardware savings. However, this can lead to catastrophic loss of numerical precision due to improper alignment caused by the redundancy inherent in unnormalized floating-point representations. 
More specifically, the first step in the add operation is establishing which operand is larger. The smaller operand is then aligned to the larger by right shifting, which can destroy significant bits of the smaller mantissa. This is acceptable, when the operand being right shifted is known to be smaller than the operand being aligned to. However, discovering which operand is smaller is very difficult when unnormalized numbers are compared, since a large exponent value may be belied by leading zeros in the mantissa. If the smaller operand is mistakenly identified as the larger operand, the larger operand may then be right shifted during the radix point alignment phase of the add operation, potentially destroying many significant bits and causing catastrophic accuracy loss. Constructing hardware that is guaranteed to correctly identify the largest operand given unnormalized formats is more expensive than using a normalized representation throughout the computation, and so unnormalized formats are not useful, despite some lingering and poorly developed thoughts still percolating in the FPGA community.

This thesis presents another way to reduce normalization costs: using a higher radix representation. In a representation with radix $2^{\nu}$, the normalization procedure is simplified. Instead of exactly locating and positioning the leading non-zero bit as required with radix 2 formats, the leading one is located and positioned only to within $\nu$ bits. This relaxation results in the hardware savings which motivate this thesis.

\subsection{Floating-Point Format Background}

\subsubsection{IEEE Format Details}

The IEEE standards mandate exact representations for binary single and double precision floating-point formats [4], as well as more flexible guidelines for singleextended and double-extended formats. Quadruple precision is not yet an official standard, although at present, an IEEE working group is standardizing it [12]. The IEEE standards have been extraordinarily successful in ensuring a level of portability for computer arithmetic across a vast array of implementations and disparate 
architectures. Since these standards are the basis for virtually all floating-point computation, it is important to understand their details.

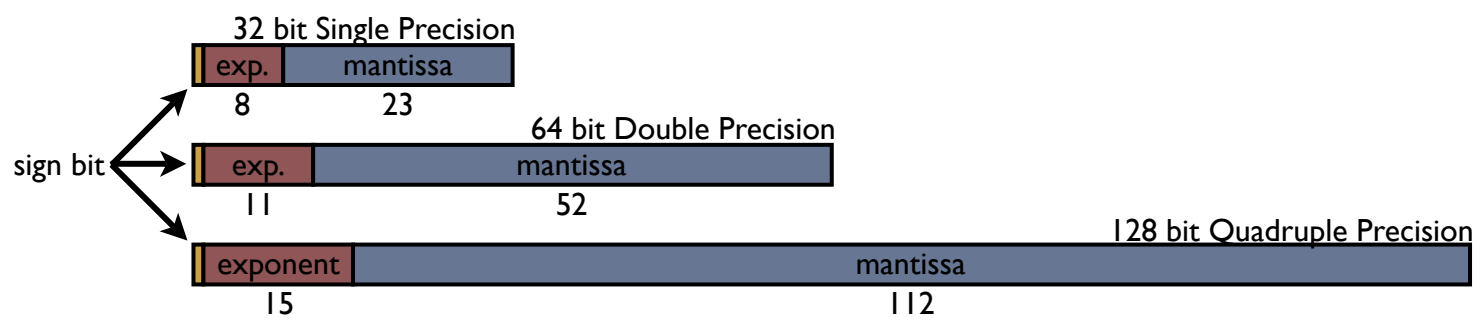

Figure 2.1: Standard Floating-Point Formats

Figure 2.1 illustrates the IEEE standard binary single and double precision floating-point formats, along with the proposed IEEE standard for quadruple precision floating-point format [12]. Single precision has 1 sign bit, 8 exponent bits, and 23 mantissa bits. The IEEE format requires normalization, and since it uses radix 2 , it is known a priori that the first bit of the mantissa is a 1, which means that it can be implied. This implied bit gives IEEE formats an extra bit of mantissa. For example, IEEE single precision has effectively 24 bits of mantissa, rather than the 23 which are expressed in the external representation as shown in Figure 2.1.

As an aside, it is worth mentioning that the vocabulary used by the IEEE standards is in a state of flux, and the cited draft of IEEE754R [12] eschews the use of the names "single", "double" and "quadruple" in favor of the more precisely descriptive labels "binary32", "binary64" and "binary128". Similarly, the the word "denormalized" has been replaced with "subnormal". In this thesis, we will use the more established terminology.

IEEE floating-point exponents are represented in biased form, where an $n$ bit exponent has bias $B I A S=2^{n-1}-1$, and the actual encoded exponent value is 
$e+B I A S$. This particular bias greatly simplifies floating-point comparison [13], and so we choose the standard bias for our higher radix representations.

Along with the biased exponent and implied leading one, another feature of IEEE standard floating-point is that the mantissa is interpreted to be within the range $[1,2)$. This means that the standard places the binary point one binary digit into the mantissa, or utilizing our earlier notation, defines $\delta=1$.

As mentioned earlier, IEEE floating-point specifies the use of denormalized numbers for representing exceptionally small numbers. If a number would be represented with an exponent value smaller than the smallest permitted exponent, gradual underflow allows leading zeros into the mantissa. Support for denormalized numbers can be expensive in hardware, but it is required for applications which require high numerical accuracy.

\subsubsection{Rounding}

The IEEE specification describes four rounding modes: Round to $+\infty$, Round to $-\infty$, Round to Zero, and Round to Nearest Even. Round to Zero is equivalent to truncation, which means it has very poor numerical performance, but requires no special hardware support. The default rounding mode is Round to Nearest Even, which is the best choice from a numerical perspective, but requires a large amount of hardware to implement correctly. The Round to $\pm \infty$ modes are used relatively rarely - originally they were intended for hardware support of interval arithmetic [14], which attempts to keep track of the uncertainty in a calculation by computing both an upper and a lower bound at each step. However, interval arithmetic is not widely used, and so most floating-point calculations default to the Round to Nearest even rounding procedure.

Table 2.1 details the rounding logic for all four rounding modes. In the table, "X" represents a "don't care" value. The "LSB" bit is the least significant bit of the mantissa after normalization. "Round" refers to the next least significant bit after the LSB. The "Sticky" bit is the logical or of all other less significant bits which were generated during the operation, as a result of alignment, multiplication, 
Table 2.1: Round Logic

\begin{tabular}{|ccccc|c|}
\hline Mode & Sign & LSB & Round & Sticky & Round Up \\
\hline$\rightarrow 0$ & $\mathrm{X}$ & $\mathrm{X}$ & $\mathrm{X}$ & $\mathrm{X}$ & 0 \\
\hline$\rightarrow+\infty$ & + & $\mathrm{X}$ & 1 & $\mathrm{X}$ & 1 \\
$\rightarrow+\infty$ & + & $\mathrm{X}$ & $\mathrm{X}$ & 1 & 1 \\
$\rightarrow+\infty$ & + & $\mathrm{X}$ & 0 & 0 & 0 \\
$\rightarrow+\infty$ & - & $\mathrm{X}$ & $\mathrm{X}$ & $\mathrm{X}$ & 0 \\
\hline$\rightarrow-\infty$ & + & $\mathrm{X}$ & $\mathrm{X}$ & $\mathrm{X}$ & 0 \\
$\rightarrow-\infty$ & - & $\mathrm{X}$ & 1 & $\mathrm{X}$ & 1 \\
$\rightarrow-\infty$ & - & $\mathrm{X}$ & $\mathrm{X}$ & 1 & 1 \\
$\rightarrow-\infty$ & - & $\mathrm{X}$ & 0 & 0 & 0 \\
\hline$\rightarrow$ even & $\mathrm{X}$ & $\mathrm{X}$ & 1 & 1 & 1 \\
$\rightarrow$ even & $\mathrm{X}$ & 0 & 1 & 0 & 0 \\
$\rightarrow$ even & $\mathrm{X}$ & 1 & 1 & 0 & 1 \\
$\rightarrow$ even & $\mathrm{X}$ & $\mathrm{X}$ & 0 & $\mathrm{X}$ & 0 \\
\hline
\end{tabular}

etc. Generating the sticky bit involves a significant amount of circuitry. However, it prevents certain calculations from drifting under iterative calculation with the Round to Nearest Even procedure, and is therefore worth the cost [15]. Finally, the "Round Up" bit is the result of the rounding logic. If it is a "1", the mantissa must be incremented to form the rounded mantissa.

\subsubsection{Treatment of Special Numbers}

Table 2.2: Special Numbers

\begin{tabular}{|l|c|c|}
\hline Special Number & $n$-bit Exponent & Mantissa \\
\hline Not a Number (NaN) & $2^{n}-1$ & $\neq 0$ \\
\hline \pm Infinity & $2^{n}-1$ & 0 \\
\hline Denormalized Number & 0 & $\neq 0$ \\
\hline \pm Zero & 0 & 0 \\
\hline
\end{tabular}


Another peculiarity of the IEEE format is the use of reserved exponent and mantissa values for special numbers. Table 2.2 details the four types of special numbers defined by the IEEE specification, which reserve the maximum $\left(2^{n}-1\right.$ for an $n$-bit exponent) and minimum (0) representable exponent values.

The reservation of the 0 exponent value for denormalized numbers and zero can be seen as a consequence of the implied bit mentioned earlier. Since denormalized numbers and zero are the only numbers in IEEE floating-point not to have a leading one, this exceptional condition is accounted for by reserving the 0 exponent value, and then not expressing the leading one when the 0 exponent value is encountered. If the leading one was not implied, any exponent could be used to represent the number zero, and the minimum exponent could be used for regular numbers as well as denormalized numbers. Practically, the dynamic range of IEEE floating-point formats has been very slightly reduced in order to provide an extra bit of precision for the mantissa.

Another subtle complication due to the implied leading one defined by IEEE formats is that denormalized numbers have an implied exponent of "1", and not "0", with which they are encoded. This is necessary to provide gradual underflow.

The specification also provides behavior for two types of Not a Number (NaN) values (quiet and signaling), as well as five exceptions (invalid operation, division by zero, overflow, underflow, and inexact). FPGA implementations tend not to implement these exactly as outlined in IEEE754, since the concept of exception and trap doesn't make sense for a non von Neumann computer such as an FPGA. Instead, FPGA implementations generally adhere to the spirit of the standard: any operation on a NaN yields a NaN, division by zero yields a correctly signed infinity, and so forth. For reference, Tables 2.3, 2.4, 2.5, and 2.6 detail the behavior of each operator to special operands.

\subsubsection{Quadruple Precision}

As mentioned previously, quadruple precision is not defined in the current IEEE specifications. This is because double precision has been adequate for many 
Table 2.3: Addition Special Cases

\begin{tabular}{|c|cccc|}
\hline+ & $-\infty$ & -0 & +0 & $+\infty$ \\
\hline$-\infty$ & $-\infty$ & $-\infty$ & $-\infty$ & NaN \\
-0 & $-\infty$ & -0 & $\pm 0^{*}$ & $+\infty$ \\
+0 & $-\infty$ & $\pm 0^{*}$ & +0 & $+\infty$ \\
$+\infty$ & $N a N$ & $+\infty$ & $+\infty$ & $+\infty$ \\
\hline
\end{tabular}

* -0 is chosen when the rounding mode is round to $-\infty$.

Otherwise, +0 is chosen.

Table 2.4: Subtraction Special Cases

\begin{tabular}{|c|cccc|}
\hline- & $-\infty$ & -0 & +0 & $+\infty$ \\
\hline$-\infty$ & $N a N$ & $-\infty$ & $-\infty$ & $-\infty$ \\
-0 & $+\infty$ & +0 & -0 & $-\infty$ \\
+0 & $+\infty$ & +0 & +0 & $-\infty$ \\
$+\infty$ & $+\infty$ & $+\infty$ & $+\infty$ & NaN \\
\hline
\end{tabular}

Table 2.5: Multiplication Special Cases

\begin{tabular}{|c|cccc|}
\hline $\mathrm{x}$ & $-\infty$ & -0 & +0 & $+\infty$ \\
\hline$-\infty$ & $+\infty$ & $N a N$ & $N a N$ & $-\infty$ \\
-0 & $N a N$ & +0 & -0 & $N a N$ \\
+0 & $N a N$ & -0 & +0 & $N a N$ \\
$+\infty$ & $-\infty$ & $N a N$ & $N a N$ & $+\infty$ \\
\hline
\end{tabular}

Table 2.6: Division Special Cases

\begin{tabular}{|c|cccc|}
\hline$/$ & $-\infty$ & -0 & +0 & $+\infty$ \\
\hline$-\infty$ & $N a N$ & $+\infty$ & $-\infty$ & $N a N$ \\
-0 & $+\infty$ & $N a N$ & $N a N$ & $-\infty$ \\
+0 & $-\infty$ & $N a N$ & $N a N$ & $+\infty$ \\
$+\infty$ & $N a N$ & $-\infty$ & $+\infty$ & $N a N$ \\
\hline
\end{tabular}


applications, and quadruple precision operators would have been prohibitively expensive to fabricate in older technologies. However, demand for greater precision is increasing, since double and double extended precisions are not adequate for some scientific applications including climate modeling, computational physics, computational geometry, fluid dynamics, computational number theory, and experimental mathematics [16], [17]. Since no commodity CPU currently implements quadruple precision, quadruple precisions are usually done in slow software routines. When higher precision and performance is required, one is forced to turn to clever tricks such as double-double representation, which uses two IEEE double precision numbers in tandem to represent a higher precision number.

Although demand for quadruple precision is increasing, it is doubtful that the mass market will ever prefer quadruple precision over increased computational throughput, which means that it is unlikely that quadruple precision units will be integrated into mass market CPUs. This, along with the extreme penalty inherent in software floating-point routines, makes quadruple precision calculations a ripe target for FPGA implementation.

Higher radix formats can provide large efficiency gains for very high precision operators, as will be shown later in this thesis. As an aside, Appendix A presents a factorization method which significantly reduces the number of embedded block multipliers required for the mantissa multiplication for very high precision floatingpoint calculations, thus enabling their implementation on multiplier limited FPGAs.

\subsection{Historical Background}

Before the advent of floating-point standards, various radices greater than 2 were in use. For example, the Illiac II used $\beta=4$, the Burroughs 5500 used $\beta=8$, and the IBM 360 used $\beta=16$ [18]. IBM mainframes still support hexadecimal floating-

point $(\beta=16)$ for compatibility reasons [19], [20]. The designers of these systems chose higher radix representations because of area and latency savings for higher radix floating-point arithmetic units, which come primarily through reductions in the 
size of the shifters and leading one detection circuitry due to relaxed normalization procedures.

During the late 1960s and early 1970s, there was tension between hardware designers and numerical analysts as to the choice of radix. Hardware designers wanted to use higher radix representations to reduce the hardware cost of floating-point functional units, and numerical analysts were set on radix 2 because of its numerical advantages. The numerical analysts won the battle, because the cost of a floatingpoint arithmetic unit decreased so quickly that hardware penalties incurred by the use of radix 2 ceased to be a concern. IEEE standard 754 mandates the use of radix 2, and although IEEE 854 is entitled "IEEE Standard for Radix-Independent Floating-Point Arithmetic", it forbids the use of radices other than $\beta=2$ and $\beta=10$ [5]. Decimal $(\beta=10)$ representations are required for financial calculations, in order to produce exactly the same results as those done by hand [21], but their inefficient implementation causes them to be avoided whenever possible.

Despite the hardware advantages of higher radix floating-point, radix 2 has been chosen as the standard over other commensurable radices because radix 2 systems always have the best numerical accuracy when given a fixed number of bits to encode the entire floating-point number, including mantissa, exponent, and sign 222. This comes about because there are no leading zeros in normalized radix 2 mantissas, which means that all mantissa bits are always significant. With higher radices of the form $\beta=2^{\nu}$, up to $\nu-1$ bits may be leading zeros. These leading zeros can be understood as exponent information which has been encoded into the mantissa, which has the effect of reducing the number of significant bits in the mantissa. Additionally, normalized radix 2 mantissas always have a leading 1, which can be implied, freeing one extra bit of precision, as mentioned earlier.

Because memory and register file oriented computing systems must represent floating-point data in a convenient, fixed number of bits, numerical accuracy per bit of representation is the dominant measure of a floating-point representation's usefulness for the general computing world. The studies which led to the choice of radix 2 as the standard were all based on this underlying premise, and so they kept 
the bitwidth of the floating-point datum constant as they determined which radix was most advantageous (e.g., [18], 22]).

In contrast to conventional computing systems, custom floating-point datapaths implemented on FPGAs are not as limited by memory concerns. Data being processed on an FPGA is more likely to stay on chip until the application has finished processing it [1]. This, along with the use of distributed state in pipeline registers instead of a central register file, frees FPGA-based computation systems from rigid restrictions on floating-point representation imposed by memory interfaces. Instead, FPGA performance is constrained by circuit area, since FPGAs gain their high performance by exploiting spatial parallelism, unrolling a computation to fill the available compute fabric. Non-standard bitwidth floating-point formats are common on FPGAs because their use may enable the implementation of a particular computation or increase performance, while still providing acceptable numerical accuracy.

Since FPGA performance is constrained by circuit area instead of memory interface, the fundamental assumption which led to the choice of radix 2 and exclusion of higher radix representations is not of primary importance. Instead of numerical accuracy per bit of representation, FPGA-based computing systems aim to maximize numerical accuracy and performance per unit of circuit area. From this perspective, higher radix representations are more efficient for FPGAs, even when their binary forms must be slightly enlarged in order to equalize numerical performance with their radix 2 counterparts.

The numerical disadvantages of higher radix representations can be resolved by adding a few bits to the mantissa, which is not practical in the general computing world because of the constraints imposed by rigid memory interfaces. For a radix $2^{\nu}$ representation, an additional $\nu-1$ bits of mantissa are sufficient to equalize worst case numerical accuracy, while providing increased average accuracy. Because FPGAs are architected with bit-level granularity, the penalty for a few extra mantissa bits is minimal. Additionally, the implied bit touted as a unique advantage of radix 2 representations saves practically no circuit area, since it must be expressed prior to 
any calculation. To prove this, we implemented a radix 2 adder with and without the implied bit and found that the area savings was $0.3-0.9 \%$, which is negligible.

In summary, the advantages of radix 2 representations which led to the rejection of higher radix representations in the past are not decisive for FPGA implementations, and the numerical disadvantages of higher radix representations can be easily overcome in FPGA implementations.

\subsection{On the Need for Bit-Identical Results}

Some people may feel that a higher radix implementation is not acceptable for FPGA designs which aim to replace an IEEE compliant CPU. Although it is true that a higher radix design will not produce bit-for-bit the same output as a standard IEEE design, the most popular floating-point units available today do not produce bit-identical results to each other. For example, the Intel x87 floating-point unit performs all calculations in an internal 80-bit double extended format, converting down to single or double precision only on command [23]. The results from an x87 FPU will thus be generally more accurate, and therefore not identical to the results from a 64-bit double precision unit which satisfies the bare minimum of the IEEE specification. Another example of a widely used, higher precision floatingpoint calculation which is not bit-for-bit identical with other IEEE 754 compliant implementations is the ubiquitous Fused Multiply-Add (FMA) unit, which computes $d=a b+c$ at once, with only one rounding operation [24]. FMA units are found on a great many processors, such as Intel's IA64, and Motorola and IBM's PowerPC [25], to name a few. Because the FMA computes two operations with only one rounding, it is more accurate than the IEEE standard requires, and therefore not bit-for-bit identical. This has not been a barrier to the success of FMA architectures, which are becoming extremely widespread.

These two examples show that the lack of bit-for-bit identical results which will result from computing with a higher radix internally and using IEEE formats externally should not pose a problem for most applications, since, as we will show, the results will have higher numerical accuracy than the standard requires. 


\subsection{Related Work}

When researching in an area as well established as floating-point arithmetic, there are a great number of publications which relate to the work. A complete bibliography is not attempted in this thesis, instead, some important papers relating to floating-point arithmetic on FPGAs as well as higher radix floating-point representations are outlined in this section.

\subsubsection{Floating-Point Arithmetic on FPGAs}

There has been much work researching floating-point implementation on FPGAs. The first mention of implementing floating-point arithmetic on FPGAs is by Fagin and Renard in 1994 [26]. An IEEE-754 compliant, single precision adder and multiplier was implemented on Actel anti-fuse based FPGAs, and the cost of pipelining, rounding and support for denormalized numbers was carefully characterized. Their design, consisting of one adder and one multiplier, was partitioned among 4 FPGAs, primarily due to the expense of the $24 \times 24$ bit mantissa multiplier. The authors concluded that FPGA density needed to improve $2-4 \mathrm{x}$ in order to fit the mantissa multiplier on a single FPGA.

In 1995, Shirazi, Walters, and Athanas reported on their floating-point adder, multiplier and reciprocal units, which operated on a custom 16 or 18 bit floatingpoint representation [27. Their work did not support any rounding mode except truncation, nor did it support denormalized numbers. Again, the conclusion was that larger representations, such as IEEE Single Precision, would require several FPGAs to implement.

Despite the low logic density of FPGAs available at the time, Louca, Cook and Johnson implemented a floating-point adder and multiplier that operated on IEEE Single Precision data [28]. Although the stated intention of their work was to maxi-

mize numerical accuracy by using full Single Precision data, they did not implement any rounding mode except truncation, nor did they implement denormalized number support, since those features were deemed too expensive. To reduce the cost of the 
mantissa multiplier, the authors used digit-serial techniques to reduce the multiplier size significantly, at the expense of a longer initiation interval - in this case, six cycles.

More recently, implementing floating-point operators on FPGAs has become practical. Besides the density increases which come due to semiconductor process improvements, FPGAs now have special architectural features designed to improve arithmetic performance. Most notable is the inclusion of embedded block multipliers in FPGAs, such as those from Xilinx, Altera, and Lattice Semiconductor [29] [30] [31], which drastically reduce the cost of the mantissa multiplier.

Taking advantage of the embedded multipliers, Roesler and Nelson found that the size of floating-point multipliers was reduced by $80 \%$ [32]. They also advocated the use of embedded multipliers for normalization shifting, as well as for mantissa multiplication. Lee and Burgess presented latency-optimized floating-point units which provided 4 cycle at $100 \mathrm{MHz}$ performance for multiplication and addition, as well as some pipelined division and multiplication operators [33].

Several libraries of floating-point operators for FPGAs are available. Govindu et al compare their own library with commercial libraries from Nallatech and Quixilica [34. They also compare themselves with the library developed by Belanovic, which can be found in [35]. Each library provides varying levels of parameterizability and compatibility with the IEEE standard.

These libraries have been utilized to implement high performance floatingpoint systems. For example, Smith and Schnore used the Nallatech library to investigate the suitability of FPGAs for acceleration of Computational Fluid Dynamics [36]. They concluded that an FPGA based Computational Fluid Dynamics accelerator would achieve between 100-200x greater sustained performance over state-of-theart processors, while requiring significantly less power. Unfortunately, their work did not address system level issues, assuming that all computation was proceeding on their Nallatech board without having to use the PCI bus. This makes their results less interesting, since system level bottlenecks often dominate performance. Still, the results were promising. 
Gokhale et al implemented a Monte Carlo Radiative Heat Transfer Simulation on a variety of Xilinx FPGAs, and showed speedups of 10x over a Pentium 4 [37]. They would have achieved greater speedups if their code had not contained data dependent loop exits, which allowed the processor to avoid many loop iterations on some loops, whereas the FPGA based calculation performed all loop iterations regardless of whether the early loop exit criteria were satisfied. Although the stated object of this research was to go beyond peak performance estimations and provide experience mapping real supercomputer type applications to FPGAs, Gokhale et al ignored system level issues completely. In fact, they assumed that all input data was initialized in block RAMs on the FPGA, and they did not take into account the time necessary to write results into the block RAMs or to the outside world when computing speedup over the conventional processor.

These results indicate that FPGA-based floating-point processors promise to deliver outstanding performance on real world applications. However, greater examination of system level issues for FPGA based accelerators is obviously warranted. The lack of published results which take these issues into account may simply be a result of the equipment and resources available to researchers, since currently available FPGA platforms are limited to the PCI bus on commodity computing systems. Although this thesis is not focused on system issues for FPGA-based floating-point datapaths, these issues are currently a significant problem which should be addressed.

\subsubsection{Higher Radix Floating-Point Implementations}

Higher radix floating-point representations have been in use for many years, as mentioned earlier, although they are not very common at present. IBM still sells mainframes which have native hexadecimal floating-point operators. The design of a native hexadecimal FPU which also operates on binary, IEEE operands is described in [20]. Their FPU is optimized for the legacy hexadecimal formats, and so operations on binary formats require extra cycles for converting IEEE data into an internal hexadecimal format, and then converting back to IEEE format after the operation is 
complete. Their conversion process is very similar to the one outlined in this thesis, except that their choice of radix point position complicates the conversion slightly.

A redundant signed hexadecimal format is used internally in [38]. The focus of that work is to reduce latency by avoiding carry propagation, however they also use a hexadecimal format internally to reduce normalization costs. Similarly to IBM, they convert to and from IEEE formats at the beginning and end of the operation, although the conversion is taken out of the critical loop latency.

Hexadecimal floating-point has been recently advocated for use in lightweight, low power ASIC designs [39], where the authors found that it reduced the size of the floating-point adder by $11 \%$, but increased the size of the multiplier by $43 \%$ for very small (14-15 bit) floating-point word sizes. Our work shows a greater benefit for hexadecimal floating-point operators because we include support for denormalized numbers, we are implementing on an FPGA fabric as opposed to an ASIC, and because we present results from larger floating-point formats (equivalent to IEEE single, double, and quadruple precision).

There have been several projects which use higher radix floating-point to reduce implementation costs. However, none of them examined the benefits of higher radix representations on FPGAs. FPGAs are uniquely suited for higher radix floating-point implementation, since the relative cost of normalization shifters is high on FPGAs. Additionally, the use of embedded block multipliers common on FPGAs masks most of the area increase from the slightly larger mantissa multiplier required in a higher radix floating-point representation. The singular strengths and weaknesses of FPGAs warrant a reexamination of the choice of floating-point radix. 


\section{Chapter 3}

\section{Proposed Representation}

\subsection{Overview}

In this thesis, we present a family of higher radix floating-point representations. Because radix 2 formats still have compelling advantages in terms of numerical performance per bit, and because of their ubiquity, we envision the need for systems which operate on and produce standard, radix 2 floating-point numbers.

Figure 3.1 shows an overview of such a system, with converters between an external radix 2 format and an internal radix 16 format. One of the main goals of our higher radix formats is maximum compatibility with standard radix 2 formats. We want them to have equivalent dynamic range, and equal worst case accuracy with radix 2 formats. We also want conversion between standard formats and internal higher radix formats to be as simple as possible.

\subsection{Radix Point Position}

Changing the radix of a floating-point representation affects both the mantissa and the exponent value of a floating-point number. Since the radix is exponentiated by the exponent value, higher radix representations need smaller values of exponent to represent the same number. If $e$ represents the exponent of a radix 2 number

which we wish to represent in a radix $\beta=2^{\nu}$ representation, it is easy to solve for the value of $e^{\prime}$ as a function of $e$ :

$$
\begin{aligned}
& 2^{e}=\beta^{e^{\prime}} \\
& 2^{e}=2^{\nu e^{\prime}}
\end{aligned}
$$




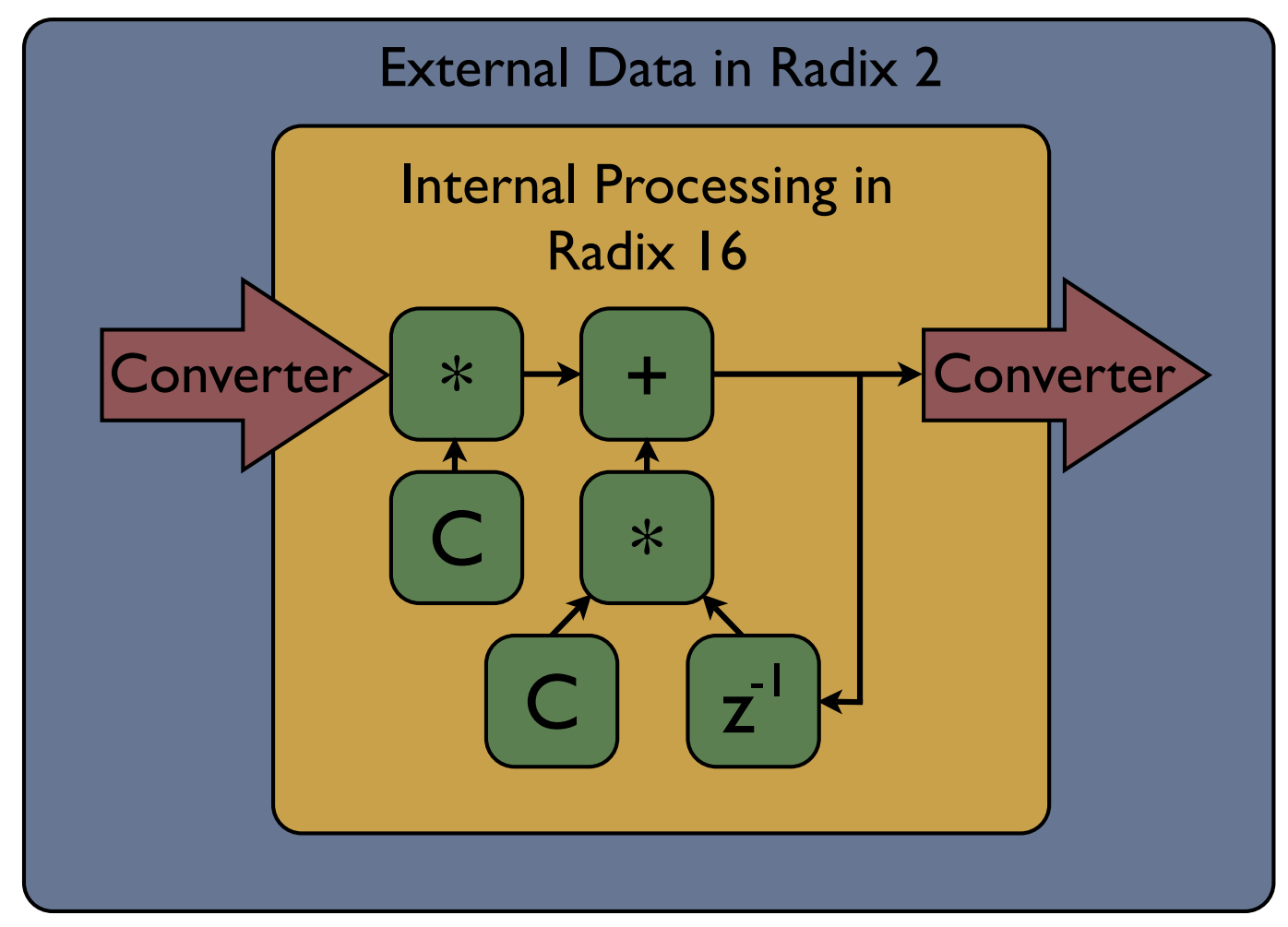

Figure 3.1: Externally Radix 2, Internally Radix 16 System

$$
e^{\prime}=\frac{e}{\nu}
$$

Thus, mapping from radix 2 to radix $2^{\nu}$ involves dividing by $\nu$. It follows that if we wish to represent the same range of numbers as the standard formats represent, the exponent values will be smaller. This means that we can restrict the allowed exponent range by a factor of $\nu$ compared to a radix 2 representation, while still keeping a dynamic range equal to that of the radix 2 representation. This allows us to represent the higher radix exponent with $\left\lfloor\log _{2} \nu\right\rfloor$ fewer bits and keep roughly the same dynamic range. Alloting fewer bits for the exponent frees up bits for the mantissa, and reduces the complexity of the exponent calculations which occur during floating-point operations. 
Also, since mapping between radix 2 and radix $2^{\nu}$ involves division, and therefore mapping between radix $2^{\nu}$ and radix 2 involves multiplication, conversion circuitry will be complicated if $\nu$ itself is not a power of 2 . If $\nu$ is a power of 2 , the multiplication and division can be done by shifting appropriately, as opposed to needing lookup tables for multiplication and division when $\nu$ is not a power of two. Thus, we are most interested in radices of the form $2^{2^{k}}$, such as 4 and 16. Larger radices which satisfy this condition, such as 256 or 65536, are less interesting for reasons which will be explained later.

In order for the exponent mapping to be accomplished by a simple shift, we must take into consideration the $\delta$ parameter, which accounts for the placement of the radix point. Specifically, we need to determine where the radix point should be placed in our higher radix format in order to allow for the simplest possible exponent conversion procedure. First we will show this mathematically, then illustrate at the bit level what needs to occur.

Let $m \in[0,1)$ be the mantissa of a radix 2 floating-point number. Let $e$ be the integer valued exponent, as encoded including bias, and let $\delta$ be the term accounting for the position of the binary point as defined earlier in Equation 2.1. Neglecting the sign for this analysis, we can represent a positive, radix 2 floating-point number as

$$
m 2^{\delta} 2^{e} .
$$

Also, let $\beta=2^{\nu}$ be the radix of a floating-point number, with mantissa $m^{\prime} \in[0,1)$ and exponent $e^{\prime}$. Let $\delta^{\prime}$ be the position of the radix point of the radix $\beta$ number, as defined earlier. A positive, radix $\beta$ floating-point number is then represented as $m^{\prime} \beta^{\delta^{\prime}} \beta^{e^{\prime}}$.

We choose

$$
e^{\prime}=\left\lfloor\frac{e}{\nu}\right\rfloor
$$

such that the radix $\beta=2^{\nu}$ exponent is formed simply by right shifting the radix 2 exponent by $\log _{2} \nu$ bits, and then truncating.

Setting the radix 2 number and the radix $\beta$ number equal to each other, and then substituting equation 3.4 into Expression 3.3 , we see that 


$$
\begin{aligned}
m^{\prime} \beta^{\delta^{\prime}} \beta^{e^{\prime}} & =m 2^{\delta} 2^{e} \\
& =m 2^{\delta} 2^{\nu\left\lfloor\frac{e}{\nu}\right\rfloor+(e \bmod \nu)} \\
& =2^{(e \bmod \nu)} m 2^{\delta} 2^{\nu\left\lfloor\frac{e}{\nu}\right\rfloor} \\
& =2^{(e \bmod \nu)} m 2^{\delta} 2^{\nu e^{\prime}} \\
m^{\prime} \beta^{\delta^{\prime}} \beta^{e^{\prime}} & =2^{(e \bmod \nu)} m 2^{\delta} \beta^{e^{\prime}} .
\end{aligned}
$$

At this point, it is easy to see that we should choose

$$
m^{\prime}=2^{(e \bmod \nu)} m
$$

In other words, the radix $\beta$ mantissa will be a shifted version of the radix 2 mantissa, and the shift amount is determined by the bits which are truncated from the radix 2 exponent when forming the radix $\beta$ exponent.

After making these choices for $e^{\prime}$ and $m^{\prime}$, we are ready to solve for $\delta^{\prime}$, which shows where the radix point of our radix $\beta$ number should be placed. Substituting into equation 3.5 .

$$
\begin{aligned}
m^{\prime} \beta^{\delta^{\prime}} \beta^{e^{\prime}} & =m^{\prime} 2^{\delta} \beta^{e^{\prime}} \\
\beta^{\delta^{\prime}} & =2^{\delta} \\
2^{\nu \delta^{\prime}} & =2^{\delta} \\
\delta^{\prime} & =\frac{\delta}{\nu}
\end{aligned}
$$

Equation 3.7 relates the radix point placement of the radix $\beta$ number to the binary point placement of the radix 2 number, when the radix $\beta$ exponent and mantissa are chosen as outlined earlier. For IEEE 754 representations, the binary point is placed one digit into the mantissa from the most significant side, leading to a mantissa which is interpreted to be in the range $[1,2)$, or equivalently, $\delta=1$. The accompanying radix point placement for our high radix format is thus determined by $\delta^{\prime}=\frac{1}{\nu}$. This is a surprising result, since $\frac{1}{\nu}$ is not an integer, meaning that the radix point should be placed in the middle of one of the radix $\beta$ digits. However, if we 


\begin{tabular}{|c|c|c|c|c|}
\hline $\begin{array}{c}\text { Number } \\
\text { Range }\end{array}$ & $\begin{array}{c}\text { Radix } 2 \\
\text { Exponent }\end{array}$ & $\begin{array}{c}\text { Biased Radix } \\
2 \text { Exponent } \\
\end{array}$ & $\begin{array}{c}\text { Biased Radix } \\
16 \text { Exponent }\end{array}$ & $\begin{array}{l}\text { Radix } 16 \\
\text { Exponent }\end{array}$ \\
\hline$[16,32)$ & 4 & 10000011 & & \\
\hline$[8,16)$ & 3 & 10000010 & & \\
\hline$[4,8)$ & 2 & 10000001 & 100000 & 1 \\
\hline$[2,4)$ & 1 & 10000000 & & \\
\hline$[1,2)$ & 0 & 01111111 & & \\
\hline$[0.5,1)$ & -1 & 01111110 & & \\
\hline$[0.25,0.5)$ & -2 & 01111101 & 11 & 0 \\
\hline$[0.125,0.25)$ & -3 & 01111100 & & \\
\hline
\end{tabular}

Figure 3.2: Exponent Mapping, $\delta^{\prime}=\frac{1}{\nu}$

\begin{tabular}{|c|c|c|c|c|}
\hline $\begin{array}{l}\text { Number } \\
\text { Range }\end{array}$ & $\begin{array}{c}\text { Radix } 2 \\
\text { Exponent }\end{array}$ & $\begin{array}{c}\text { Biased Radix } \\
2 \text { Exponent } \\
\end{array}$ & $\begin{array}{c}\text { Biased Radix } \\
16 \text { Exponent }\end{array}$ & $\begin{array}{l}\text { Radix } 16 \\
\text { Exponent } \\
\end{array}$ \\
\hline$[8,16)$ & 3 & 10000010 & \multirow{4}{*}{100000} & \multirow{4}{*}{1} \\
\hline$[4,8)$ & 2 & 10000001 & & \\
\hline$[2,4)$ & 1 & 10000000 & & \\
\hline$[1,2)$ & 0 & 01111111 & & \\
\hline$[1 / 2,1)$ & -1 & 01111110 & \multirow{4}{*}{011111} & \multirow{4}{*}{0} \\
\hline$[1 / 4,1 / 2)$ & -2 & 01111101 & & \\
\hline$[1 / 8,1 / 4)$ & -3 & 01111100 & & \\
\hline$[1 / 16,1 / 8)$ & -4 & 01111011 & & \\
\hline
\end{tabular}

Figure 3.3: Exponent Mapping, $\delta^{\prime}=0$ 
expand the radix $\beta$ digits into their binary form, we see that the radix point should be placed identically to its IEEE counterpart: 1 bit into the mantissa. This means that the mantissa for our higher radix format will be interpreted to be within the range $\left[\frac{2}{\beta}, 2\right)$.

Figure 3.2 illustrates this exponent mapping process for a conversion between a radix 2 representation with 8 bits of exponent and a radix $16=2^{2^{2}}$ representation with 6 bits of exponent: the upper 6 bits of the radix 2 exponent become the radix 16 exponent. The information from the truncated exponent bits is then encoded by introducing up to $\nu-1$ leading zeros into the higher radix mantissa.

This choice of radix point placement is unorthodox: other higher-radix floatingpoint representations such as the hexadecimal formats used by IBM [19], or the CMU lightweight floating-point project [39], place the radix point to the left of the mantissa, or equivalently, choose $\delta=0$. This choice leads to a more complicated exponent mapping, as shown by Figure 3.3 . With this choice of radix point, the higher radix exponent can not be generated by choosing $e^{\prime}=\left\lfloor\frac{e}{\nu}\right\rfloor$, which is the simplest way to generate $e^{\prime}$ in hardware. Instead, the choice of radix point illustrated in Figure 3.3 leads to choosing $e^{\prime}=\left\lfloor\frac{e}{\nu}\right\rfloor+i$, where $i$ is an indicator variable which is zero unless $e \bmod \nu=\nu-1$, in which case it has the value "1". Our desire to interface cleanly with IEEE standard formats leads us to interrupt the first $\beta$-ary digit with the radix point, and choose $\delta^{\prime}=\frac{1}{\nu}$.

\subsection{Encoding}

Now that we have explained how the radix point should be placed, we can illustrate how changing the radix affects bit-level encoding. The first row of table 3.1 shows how the number 26.0 is encoded in a radix 2 representation with 4 bits of exponent and 4 bits of mantissa, explicitly showing the leading one of the mantissa that is usually implicit. The second row shows how the same number is encoded in radix 16 with 4 bits of mantissa and 2 bits of exponent, given the binary point is placed as we described earlier. Notice that in this case, no precision is lost, and both systems are able to exactly represent the number. 
Table 3.1: Encoded Numbers in Different Representations

\begin{tabular}{|l|c|c||c|c|}
\hline Representation & Desired Value & Represented Value & Exponent & Mantissa \\
\hline \hline$S_{2}^{4}, 4$ bit exponent & 26.0 & 26.0 & 1011 & 1.101 \\
\hline$S_{16}^{1}, 2$ bit exponent & 26.0 & 26.0 & 10 & 1.101 \\
\hline \hline$S_{2}^{4}, 2$ bit exponent & 3.25 & 3.25 & 1000 & 1.101 \\
\hline$S_{16}^{1}, 2$ bit exponent & 3.25 & 2.0 & 10 & 0.001 \\
\hline$S_{16}^{1.75}, 2$ bit exponent & 3.25 & 3.25 & 10 & 0.001101 \\
\hline
\end{tabular}

The third row of the table shows how the number 3.25 is encoded in the example radix 2 representation. Row 4 shows how encoding 3.25 in the hexadecimal representation causes precision to be lost. Since 3 leading zeros were introduced, the bottom 3 significant bits of the mantissa were lost, leading to a significant representation error - instead of 3.25 as desired, we end up with 2.0. This is the numerical problem that led to the rejection of higher radix formats in the past.

Row 5 shows how adding an additional 3 bits to the mantissa is sufficient for the hexadecimal representation to capture all the precision of its binary counterpart - since the worst possible scenario for hexadecimal floating point introduces 3 leading zeros, if the mantissa is extended by 3 bits, every number representable in binary floating-point is exactly represented in hexadecimal format.

As mentioned earlier, the biggest weakness of higher-radix floating-point representations is the lower accuracy per bit, or equivalently, the larger representations required to provide the same numerical performance as a radix 2 representation. Examining this penalty, table 3.2 illustrates how the overall floating-point word size changes as a function of radix, while keeping worst case accuracy and dynamic range equal or better to radix 2, taking into account the loss of the implied leading bit, the reduction in exponent size, and the expansion of the mantissa which come with higher radix representations. Figure 3.4 shows this effect graphically. Note that radix 16 is particularly advantageous, since it has the same word size as radix 8 , but gets more hardware benefit. An extension of the floating-point word by two bits, which is required for radix 8 and radix 16 formats, is not a large obstacle internally to the 
Table 3.2: Floating-point Word Size with Equalized Numeric Performance

\begin{tabular}{|c|c|}
\hline Radix & Floating-Point Word Size \\
\hline 2 & $n$ bits \\
\hline 4 & $n+1$ bits \\
\hline 8 & $n+2$ bits \\
\hline 16 & $n+2$ bits \\
\hline 256 & $n+6$ bits \\
\hline$\beta=2^{\nu}$ & $n+\log _{2} \beta-\left\lfloor\log _{2} \log _{2} \beta\right\rfloor$ \\
\hline
\end{tabular}

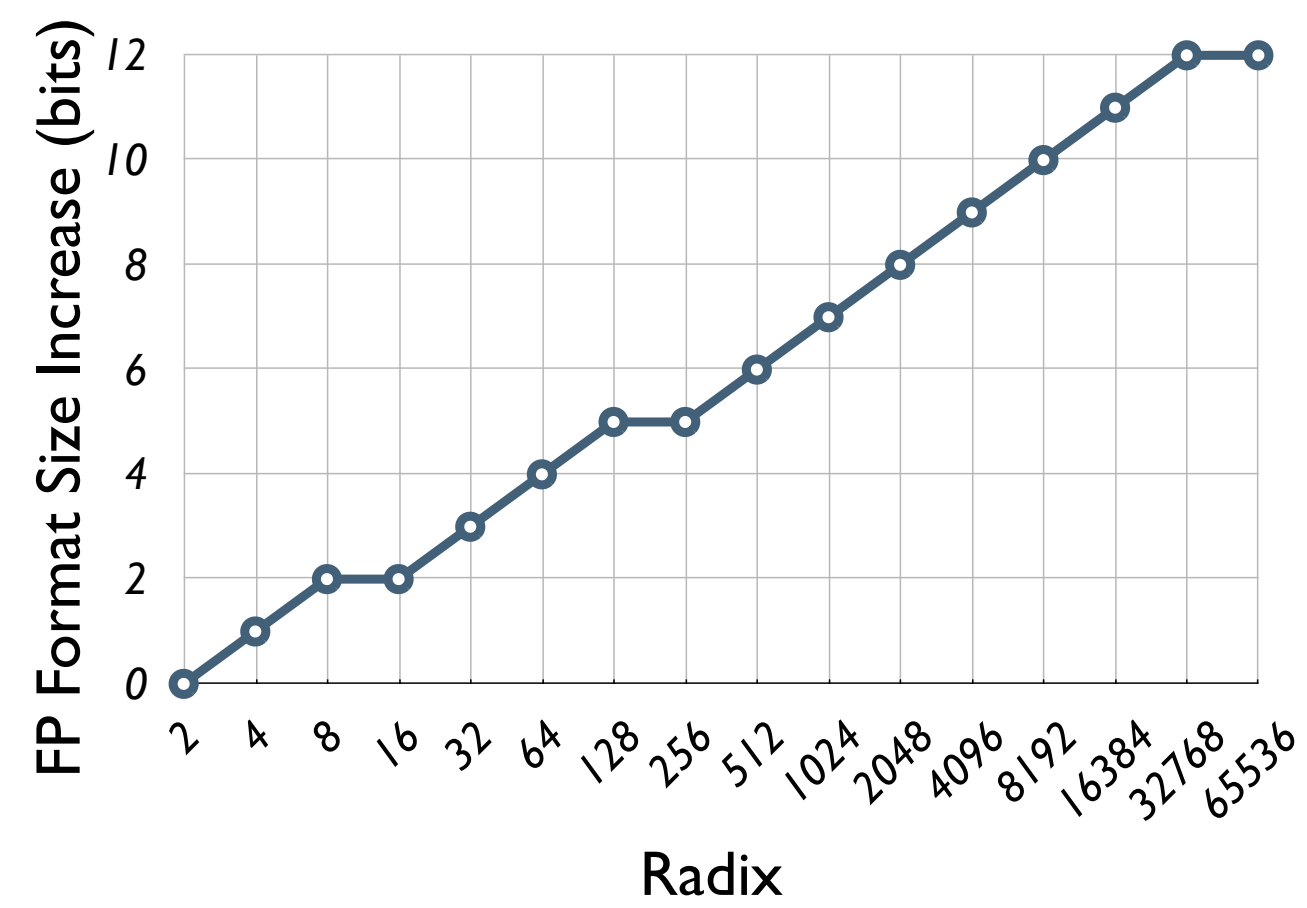

Figure 3.4: Floating-Point Format Size Increase versus Radix 
FPGA. Other established floating-point formats for use internally in FPGA-based calculation also extend the representation by two bits, which is allowable because of the bit-level granularity of FPGA fabrics, as well as the slightly wider embedded memories found on contemporary FPGAs.

\subsection{Flag Bits}

Testing whether a floating-point operand belongs to one of the IEEE special number classes is relatively expensive: it requires a full mantissa width nor gate to determine whether or not the mantissa is zero, as well as full exponent width nor and and gates to determine whether the exponent is at an extreme.

Table 3.3: Standard Special Case Logic

\begin{tabular}{|c|c|c|c|}
\hline and(exponent) & nor(exponent) & nor (mantissa) & Number Type \\
\hline 0 & 0 & 0 & Normal \\
\hline 0 & 0 & 1 & X (Disallowed) \\
\hline 0 & 1 & 0 & Denormal \\
\hline 0 & 1 & 1 & Zero \\
\hline 1 & 0 & 0 & Infinity \\
\hline 1 & 0 & 1 & NaN \\
\hline 1 & 1 & 0 & $\mathrm{X}$ (Disallowed) \\
\hline 1 & 1 & 1 & $\mathrm{X}$ (Disallowed) \\
\hline
\end{tabular}

Table 3.3 shows the logic which is usually used to determine the type of a floating-point number. This method requires that the operands be examined at the beginning of every operation. We borrow an idea from [40], which was used by Gokhale et al in [37], in which two flag bits are appended to the floating-point word which carry the special case information, although the meaning of our flag bits is slightly different than those cited.

Implying the leading bit for a radix 2 number saves practically no hardware, since it must be expressed prior to any calculation. In our internal format, the leading 
Table 3.4: Encoded Flags

\begin{tabular}{|c|c|}
\hline Flag Bits & Meaning \\
\hline 00 & Normal or Denormal number \\
\hline 01 & Zero \\
\hline 10 & Infinity \\
\hline 11 & NaN \\
\hline
\end{tabular}

bit is always expressed. This means that we don't need to distinguish between a normal or denormal number, since the only difference between them is the presence of the leading one bit. It also means that zero can have any exponent value, and is indicated by the zero flag and a zero mantissa. The two flag bits and their meaning is illustrated in figure 3.4 .

It is worth noting that the overall internal hexadecimal format, with flag bits, mantissa expansion, and exponent contraction, still fits inside of internal FPGA memories. The embedded memories in Xilinx and Altera FPGAs can be configured in multiples of 18 bits wide [29], [30]. The internal hexadecimal single precision format is 36 bits, which easily accomodated in the embedded memory on the FPGA. This is similar to the Nallatech internal format, which is a radix 2 format with the mantissa and exponent extended by 1 bit each and 2 flag bits, which is also 36 bits for single precision [36].

\subsection{Dynamic Range}

Changing the radix does impact dynamic range, although our choice of radix point position was designed to minimize this impact. To analyze this effect, we note that the mantissa is interpreted as being in the range $\left[\beta^{\delta-1}, \beta^{\delta}\right)$, when $\delta$ accounts for the placement of the radix point. The maximum representable number is then formed by multiplying the maximum mantissa $\left[\beta^{\delta}\left(1-2^{-t}\right)\right]$, where $t$ is the number of bits in the mantissa, by the maximum allowed exponent $e_{\max }$ :

$$
x_{\max }=\beta^{\delta}\left(1-2^{-t}\right) \beta^{e_{\max }}=\left(1-2^{-t}\right) \beta^{e_{\max }+\delta}
$$


The maximum allowed exponent $e_{\max }$ for an $n$-bit value, with bias $2^{n-1}-1$, and reserving the maximum possible exponent for Infinity and $\mathrm{NaNs}$ is

$$
e_{\operatorname{maxIEEE}}=2^{n}-2-\left(2^{n-1}-1\right)=2^{n-1}-1 .
$$

However, our use of flag bits allows us to avoid reserving the maximum possible exponent for Infinity and Nan, making

$$
e_{\text {maxInternal }}=2^{n-1}
$$

Similarly, the minimum representable number without going into denormalized numbers is formed by multiplying the minimum mantissa $\left[\beta^{\delta-1}\right]$ by the minimum allowed exponent $e_{\min }$ :

$$
x_{\text {min }}=\beta^{\delta-1} \beta^{e_{\text {min }}} .
$$

The minimum allowed exponent $e_{\min }$ for an $n$-bit value, with bias $2^{n-1}-1$, and reserving the minimum possible exponent for denormalized numbers and zero is

$$
e_{\operatorname{minIEEE}}=1-\left(2^{n-1}-1\right)=-2^{n-1}+2
$$

Since higher radix formats do not need to reserve an exponent value to reserve those numbers without a leading one bit, since the leading bit must be expressed, the minimum possible exponent value is

$$
e_{\text {minInternal }}=-2^{n-1}+1 \text {. }
$$

Table 3.5 shows the important parameters of our Single Precision formats at various radices. Table 3.6 shows how these parameters translate into the maximum and minimum representable numbers in these formats. The minimum representable numbers shown are still fully normalized numbers, denormalized numbers are not shown. The important thing to note is that the higher radix formats have greater dynamic range than IEEE Single Precision. Radix 8 has an especially wide range, since $8 \neq 2^{2^{k}}$, it will never have a dynamic range close to radix 2 - it will always be either greater or smaller by a factor of $\frac{3}{2}$. 
Table 3.5: Format Parameters

\begin{tabular}{|l|c|c|c|c|c|}
\hline Representation & $t$ & $n$ & $\delta$ & $e_{\max }$ & $e_{\min }$ \\
\hline \hline IEEE Single Precision & 24 & 8 & 1 & 127 & -126 \\
\hline Radix 4 Single Precision & 25 & 7 & $\frac{1}{2}$ & 64 & -63 \\
\hline Radix 8 Single Precision & 26 & 7 & $\frac{1}{3}$ & 64 & -63 \\
\hline Radix 16 Single Precision & 27 & 6 & $\frac{1}{4}$ & 32 & -31 \\
\hline \hline IEEE Double Precision & 53 & 11 & 1 & 1023 & -1022 \\
\hline Radix 4 Double Precision & 54 & 10 & $\frac{1}{2}$ & 512 & -511 \\
\hline Radix 8 Double Precision & 55 & 10 & $\frac{1}{3}$ & 512 & -511 \\
\hline Radix 16 Double Precision & 56 & 9 & $\frac{1}{4}$ & 256 & -255 \\
\hline \hline IEEE Quadruple Precision & 113 & 15 & 1 & 16383 & -16382 \\
\hline Radix 4 Quadruple Precision & 114 & 14 & $\frac{1}{2}$ & 8192 & -8191 \\
\hline Radix 8 Quadruple Precision & 115 & 14 & $\frac{1}{3}$ & 8192 & -8191 \\
\hline Radix 16 Quadruple Precision & 116 & 13 & $\frac{1}{4}$ & 4096 & -4095 \\
\hline
\end{tabular}

Table 3.6: Dynamic Range

\begin{tabular}{|l|c|c|}
\hline Representation & Maximum & Minimum \\
\hline \hline IEEE Single Precision & $3.40282234664 * 10^{38}$ & $1.1754943508 * 10^{-38}$ \\
\hline Radix 4 Single Precision & $6.8056471356 * 10^{38}$ & $5.8774717541 * 10^{-39}$ \\
\hline Radix 8 Single Precision & $1.2554203284 * 10^{58}$ & $3.1861838223 * 10^{-58}$ \\
\hline Radix 16 Single Precision & $6.8056472877 * 10^{38}$ & $5.8774717541 * 10^{-39}$ \\
\hline \hline IEEE Double Precision & $1.79769313487 * 10^{308}$ & $2.2250738585 * 10^{-308}$ \\
\hline Radix 4 Double Precision & $3.595386269725 * 10^{308}$ & $1.112536929254 * 10^{-308}$ \\
\hline Radix 8 Double Precision & $4.820624853842 * 10^{462}$ & $8.297679494417 * 10^{-463}$ \\
\hline Radix 16 Double Precision & $3.595386269725 * 10^{308}$ & $1.112536929254 * 10^{-308}$ \\
\hline \hline IEEE Quadruple Precision & $1.189731495357 * 10^{4932}$ & $3.362103143112 * 10^{-4932}$ \\
\hline Radix 4 Quadruple Precision & $2.379462990714 * 10^{4932}$ & $1.681051571556 * 10^{-4932}$ \\
\hline Radix 8 Quadruple Precision & $2.595394820897 * 10^{7398}$ & $1.541191331582 * 10^{-7398}$ \\
\hline Radix 16 Quadruple Precision & $2.379462990714 * 10^{4932}$ & $1.681051571556 * 10^{-4932}$ \\
\hline
\end{tabular}




\subsection{Numerical Accuracy}

Since this work proposes a return to floating-point representations that were rejected years ago due to numerical accuracy issues, the numerical accuracy of higher radix representations must be examined in order to understand the effects of higher radix floating-point representations on numerical accuracy.

\subsubsection{Worst Case Relative Error}

The closest exactly representable floating-point number to a real number $x$ is denoted as $\mathrm{fl}(x)$. The worst case relative error $\epsilon$ for a floating-point number representation made in approximating a real number $x$ by $\mathrm{fl}(x)$ is defined [18] as

$$
\epsilon=\sup _{x_{\min } \leq x \leq x_{\max }}\left|\frac{x-\mathrm{fl}(x)}{x}\right| .
$$

For a floating-point system with $\beta=2^{\nu}, u$ bits of mantissa, and utilizing rounding instead of truncation, it can be shown [18] that

$$
\epsilon=2^{\nu-u-1} .
$$

Equalizing the worst case error of a radix 2 system with the worst case error of a $\operatorname{radix} \beta=2^{\nu}$ system,

$$
\begin{aligned}
2^{1-u-1} & =2^{\nu-u^{\prime}-1} \\
u^{\prime} & =u+\nu-1,
\end{aligned}
$$

we see that adding an extra $\nu-1$ bits to the mantissa of a radix $\beta=2^{\nu}$ representation equalizes the worst case relative error. Intuitively, this makes sense because moving to a higher radix essentially encodes exponent information from a radix 2 representation into leading zeros in the mantissa of the higher radix representation. Since there can be up to $\nu-1$ leading zeros in a normalized $\beta=2^{\nu}$ number, adding $\nu-1$ bits to the mantissa ensures that no significant bits from the radix 2 representation will be lost in the conversion to radix $\beta$. 
We can also apply equation 3.14 to examine the relative worst case accuracy $\frac{\epsilon_{2}}{\epsilon_{1}}$ for two significance spaces $S_{\beta}^{u}$ (with worst case error $\epsilon_{1}$ ) and $S_{\phi}^{r}$ (with worst case error $\left.\epsilon_{2}\right)$ :

$$
\frac{\epsilon_{2}}{\epsilon_{1}}=2^{(1-r) \log _{2} \phi-(1-u) \log _{2} \beta}
$$

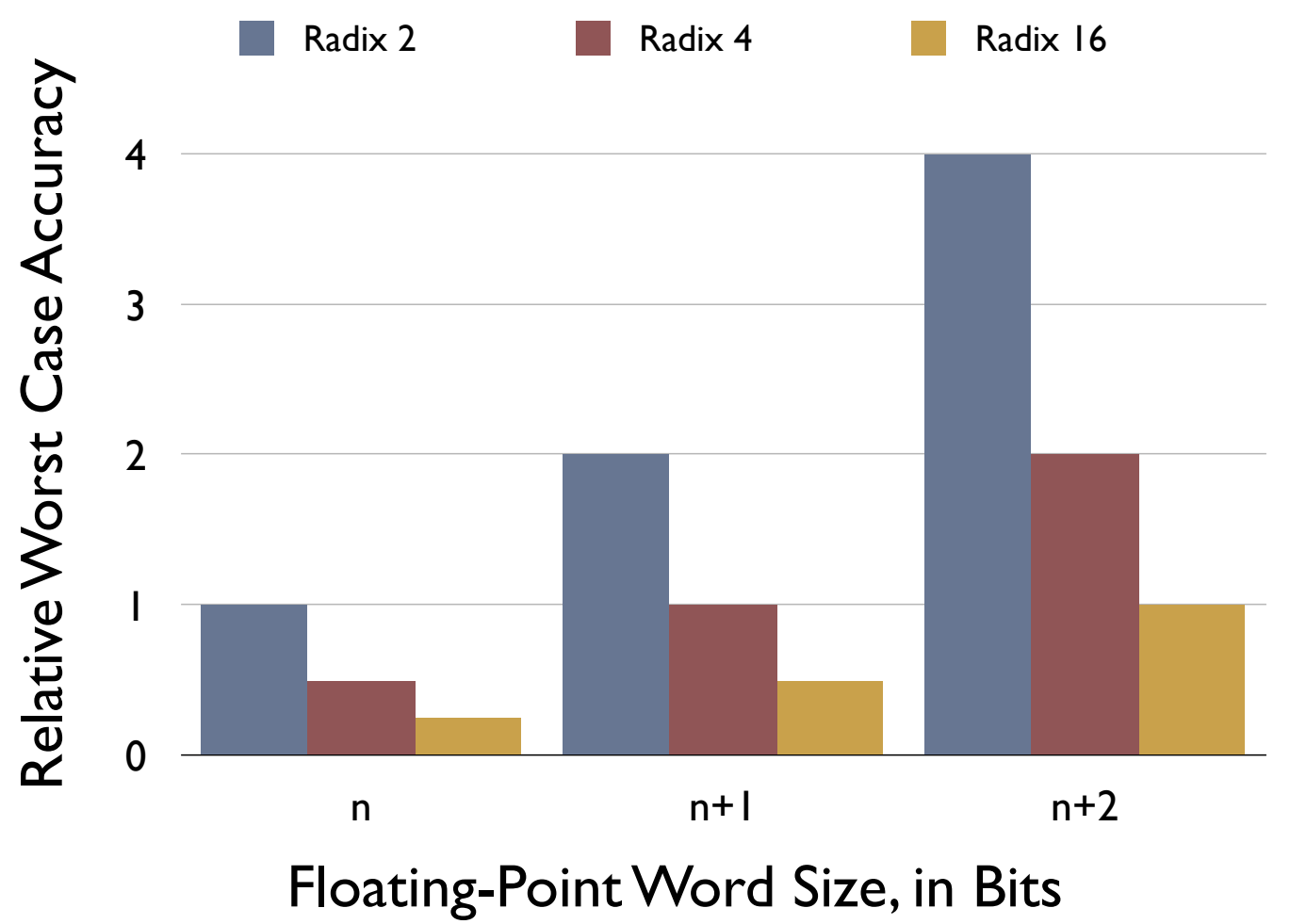

Figure 3.5: Relative Worst Case Accuracy versus FP Word Size

Figure 3.5 shows how worst case accuracy scales as the mantissa width is extended for radices 2, 4, and 16. For example, at equal word size, the radix 16 format has $\frac{1}{4}$ the worst case accuracy as the standard radix 2 format. Taking Single Precision word sizes as a concrete example, the radix 2 format has 24 effective mantissa bits, taking into account the implied leading one bit unique to radix 2 . It has 8 exponent bits, and one sign bit, leading to an overall word size of $(24-1)+8+1=32$ bits. The 
radix 16 format which fits into 32 bits overall has 25 mantissa bits, 6 exponent bits, and one sign bit. Substituting these parameters into Equation 3.17, we see that the radix 16 format has $\frac{1}{4}$ the relative worst case accuracy of the radix 2 format, at equal word size. When the word size is extended by 1 bit, the radix 4 format has the same relative worst case accuracy as the radix 2 format. Similarly, when the word size is extended by 2 bits, the radix 16 format has the same relative worst case accuracy as the radix 2 format.

\subsubsection{Relative Significance Space Density}

When $\nu-1$ bits are added to the mantissa, worst case accuracy is equalized, but average accuracy is improved. This is illustrated by the relative significance space density of the higher radix representation with an extra $\nu-1$ bits of mantissa, and the standard radix 2 representation with $u$ bits of mantissa. Relative significance space density is a measure of ratio of the number of members in 2 significance spaces. Matula found [10] that for two significance spaces $S_{\beta}^{u}$ and $S_{\phi}^{r}$, the relative significance space density is

$$
\left|\frac{S_{\phi}^{r}}{S_{\beta}^{u}}\right|=\frac{(\phi-1) \phi^{r-1}}{(\beta-1) \beta^{u-1}} \log _{\phi} \beta .
$$

Matula's derivation of this formula assumed that the number of mantissa digits $(u$ or $r$ ) was an integer. Since this work violates his assumption, his proof is restated here, with additional justification as to why it is still valid for representations with a non-integral number of $\beta$-ary digits, but an integral number of bits.

Letting $|S|$ denote the number of members of the set $S$, it is desirous to show that

$$
\lim _{M \rightarrow \infty}\left|\frac{\left\{d \mid d \in S_{\beta}^{u}, \frac{1}{M} \leq d \leq M\right\}}{\left\{b \mid b \in S_{\phi}^{r}, \frac{1}{M} \leq b \leq M\right\}}\right|=\frac{(\phi-1) \phi^{r-1}}{(\beta-1) \beta^{u-1}} \log _{\phi} \beta .
$$

To prove this, first note that the closed interval $\left[\frac{1}{M}, M\right]$ may be divided into $2\left\lfloor\log _{\beta} M\right\rfloor$ disjoint, half-open, half-closed intervals of the form $\left[\beta^{j}, \beta^{j+1}\right)$ and two subintervals of such intervals. These intervals correspond to regions of constant exponent value of the floating-point number. 
Each of these intervals contains $(\beta-1) \beta^{u-1}$ unique numbers. This follows by noting that the most significant digit of a normalized $\beta$-ary mantissa is in the range $[1, \beta-1]$, meaning that there are $\beta-1$ unique most significant digits. At this point, we recall that the number of digits $u$ is not necessarily an integer, but that the number of bits in the mantissa $\nu u$ is an integer. This allows us to expand the remainder of the mantissa, which is of length $\nu u-\nu$ bits, into binary, where we see that there are $2^{(\nu u-\nu)}$ unique values of the remainder of the mantissa. Since $2^{\nu(u-1)}=\beta^{(u-1)}$, there are then $(\beta-1) \beta^{u-1}$ unique mantissa values in each interval with constant exponent value.

Applying these facts, we see that for $M \geq 1$,

$$
\begin{aligned}
\left|\left\{b \mid b \in S_{\beta}^{u}, \frac{1}{M} \leq b \leq M\right\}\right| & =\left(2\left\lfloor\log _{\beta} M\right\rfloor+\varepsilon\right)(\beta-1) \beta^{u-1} \quad 0 \leq \varepsilon \leq 2 \\
& =\left(2 \log _{\beta} M+\varepsilon^{\prime}\right)(\beta-1) \beta^{u-1} \quad\left|\varepsilon^{\prime}\right| \leq 2
\end{aligned}
$$

The last step follows from removing the $\lfloor\cdot\rfloor$ function.

Substituting, we see that

$$
\begin{aligned}
\lim _{M \rightarrow \infty}\left|\frac{\left\{d \mid d \in S_{\beta}^{u}, \frac{1}{M} \leq d \leq M\right\}}{\left\{b \mid b \in S_{\phi}^{r}, \frac{1}{M} \leq b \leq M\right\}}\right| & =\lim _{M \rightarrow \infty} \frac{\left(2 \log _{\phi} M+\varepsilon_{2}\right)(\phi-1) \phi^{r-1}}{\left(2 \log _{\beta} M+\varepsilon_{1}\right)(\beta-1) \beta^{u-1}} \quad\left|\varepsilon_{1}\right|,\left|\varepsilon_{2}\right| \leq 2 \\
& =\lim _{M \rightarrow \infty} \frac{\frac{1}{M \log \phi}(\phi-1) \phi^{r-1}}{\frac{1}{M \log \beta}(\beta-1) \phi^{u-1}} \\
& =\frac{(\phi-1) \phi^{r-1}}{(\beta-1) \beta^{u-1}} \log _{\phi} \beta
\end{aligned}
$$

Equation 3.21 follows from taking the limit and applying l'Hôpital's rule. Equation 3.22 shows that Matula's formula does indeed still hold, despite the use of non-integer digit length mantissas.

Illustrating the meaning of equation 3.22, figure 3.6 shows the 16 members of $S_{2}^{3}$ and the 60 members of $S_{16}^{1.5}$ over the interval $[2,32)$. According to equation 3.18, $\left|\frac{S_{2}^{3}}{S_{16}^{1.5}}\right|=3.75$, and indeed we see that the ratio of the number of members of those two significance spaces over this range is $\frac{60}{16}=3.75$. From this figure, we can see three interesting things: although the mantissa of the radix 16 representation has 3 more bits than the radix 2 representation, the radix 16 representation has only 
$3.75 \mathrm{x}$ as many representable numbers as the radix 2 representation, instead of $8 \mathrm{x}$ more as one might expect. This occurs because of the 3 leading zeros which may occur in a radix 16 mantissa. When there are $\nu-1$ leading zeros, the $\nu-1$ added bits are being used to hold information which is exactly representable in the radix 2 mantissa. This occurs at the smaller end of the range of numbers representable with a given exponent. Larger numbers representable with the same exponent will have fewer leading zeros, and so the extra mantissa bits will be able to encode numbers not exactly representable in radix 2. Figure 3.6 illustrates this phenomenon over a range where the radix 16 exponent is constant. Secondly, although there are regions where the radix 16 representation is much more dense than the radix 2 representation, worst case density is exactly equal, meaning that the 3 extra bits we added equalized worst case relative error, as we showed earlier. Thirdly, all radix 2 numbers are exactly representable in the radix 16 representation, which makes conversion from radix 2 to radix 16 easier. However, converting from radix 16 back to radix 2 will require rounding because there are many numbers exactly representable in radix 16 which aren't representable in standard radix 2 representation.

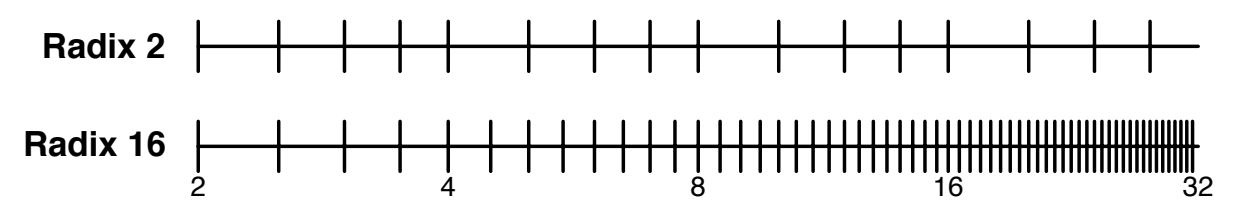

Figure 3.6: Density Comparison

The observation that adding 3 bits of mantissa makes radix 16 representation more dense than the corresponding radix 2 representation can be generalized for any radix $>2$. A radix $\beta=2^{\nu}$ representation with $t+\nu-1$ bits has $u=\frac{t+\nu-1}{\nu} \beta$-ary digits. It is easy to prove that relative significance space density of such a representation 
and the radix 2 system with $t$ bits of mantissa is greater than 1 , meaning that the higher radix system can represent more numbers than the radix 2 system:

$$
\begin{aligned}
\left|\frac{S_{2^{\nu}}^{\frac{t+\nu-1}{\nu}}}{S_{2}^{t}}\right| & =\frac{\left(2^{\nu}-1\right)\left(2^{\nu}\right)^{\frac{t+\nu-1}{\nu}}-1}{(2-1) 2^{t-1}} \log _{2^{\nu}} 2 \\
& =\frac{\left(2^{\nu}-1\right)\left(2^{\nu}\right)^{\frac{t-1}{\nu}}}{2^{t-1}} \frac{1}{\nu} \\
& =\frac{2^{\nu}-1}{\nu}
\end{aligned}
$$

Since

$$
\forall \nu>1, \frac{2^{\nu}-1}{\nu}>1
$$

the relative significance space density of the higher radix representation is greater than that of the radix 2 representation.

As we illustrated earlier, a radix 16 system with 3 extra bits of mantissa has

$\frac{2^{4}-1}{4}=3.75$ times as many exactly representable numbers as does the corresponding radix 2 system. Since the rounding schemes ensure that the closest element of $S$ to the exact result of the computation is selected as the output of that computation, the denser significance space translates into better accuracy.

Figure 3.7 shows relative significance space densities as a function of overall floating-point word size. When the floating-point word is extended by just one bit, hexadecimal formats enjoy almost a 2:1 density advantage over standard radix 2 formats, even though worst case accuracy is still less than the radix 2 formats. When the floating-point word is extended by two bits, hexadecimal floating-point has 3.75 times greater density, as mentioned earlier. It is also interesting to note that hexadecimal formats enjoy significantly greater density than radix 4 formats at the same floating-point word size.

\subsubsection{Gap Functions}

Gap functions provide a more detailed look at the relative accuracy of two number representations over an interval [10]. Given a number $x \in S$, we define its 


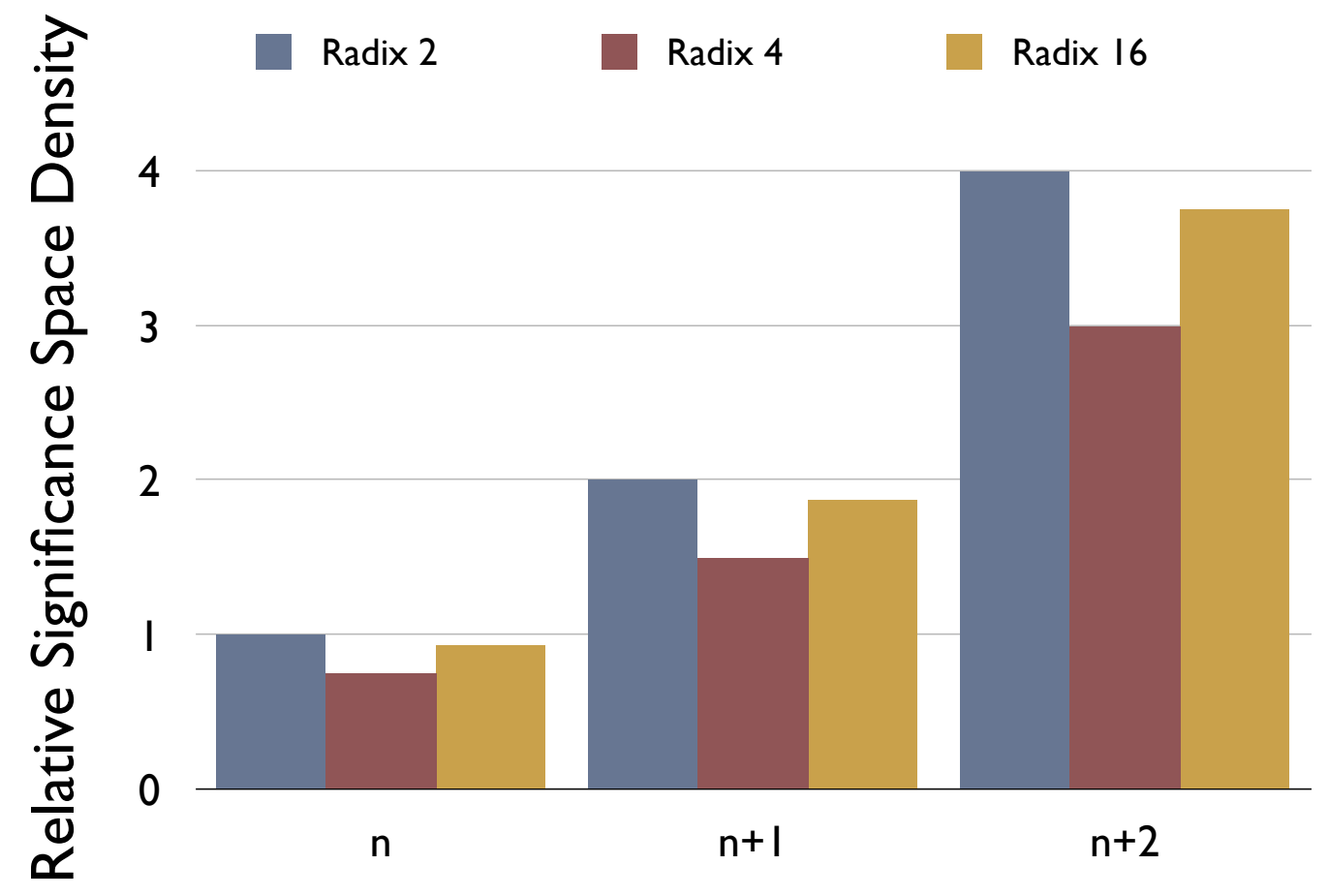

Floating-Point Word Size, in Bits

Figure 3.7: Relative Significance Space Density versus FP Word Size

successor $x^{\prime}$ to be the next largest element of $S$. The gap function for $S_{\beta}^{t}$ is defined as

$$
\gamma_{\beta}^{t}(x)=\frac{x^{\prime}-x}{x} \quad(\forall x>0)
$$

As an example, figure 3.8 shows the gap functions for a 32-bit, unsigned fixedpoint representation which represents numbers from $[0,1)$, and 32-bit, IEEE single precision floating-point. The floating-point representation is obviously much more flexible, since it represents both positive and negative numbers, with magnitudes ranging from $1.17549 * 10^{-38}$ to $3.40282 * 10^{38}$, whereas the fixed-point representation can only represent positive numbers ranging from $[0,1)$. Outside of this very narrow range, the gap function for the fixed-point representation is infinite.

Besides the much larger dynamic range of the equivalent floating-point representation, the gap functions show that normalized floating-point representations 


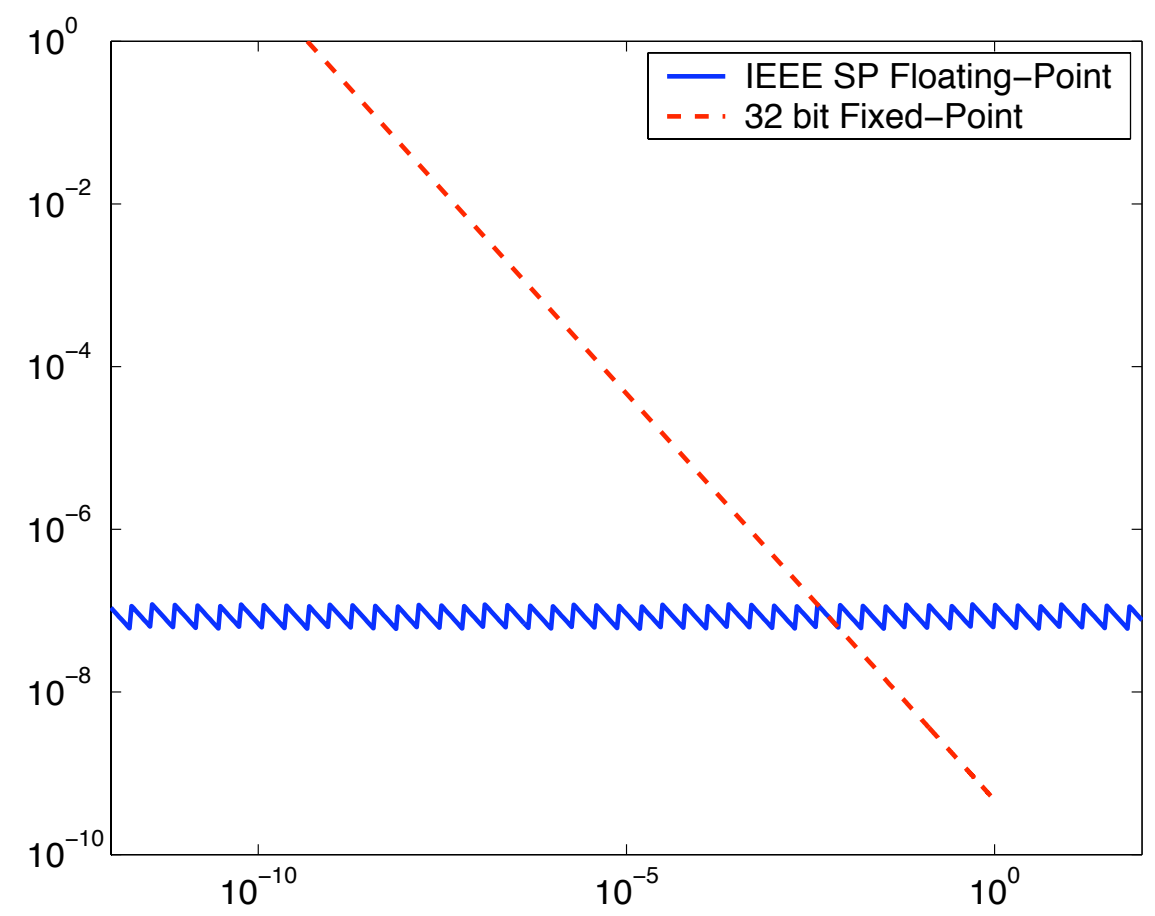

Figure 3.8: Gap functions for 32-bit Fixed-Point and 32-bit Floating-Point

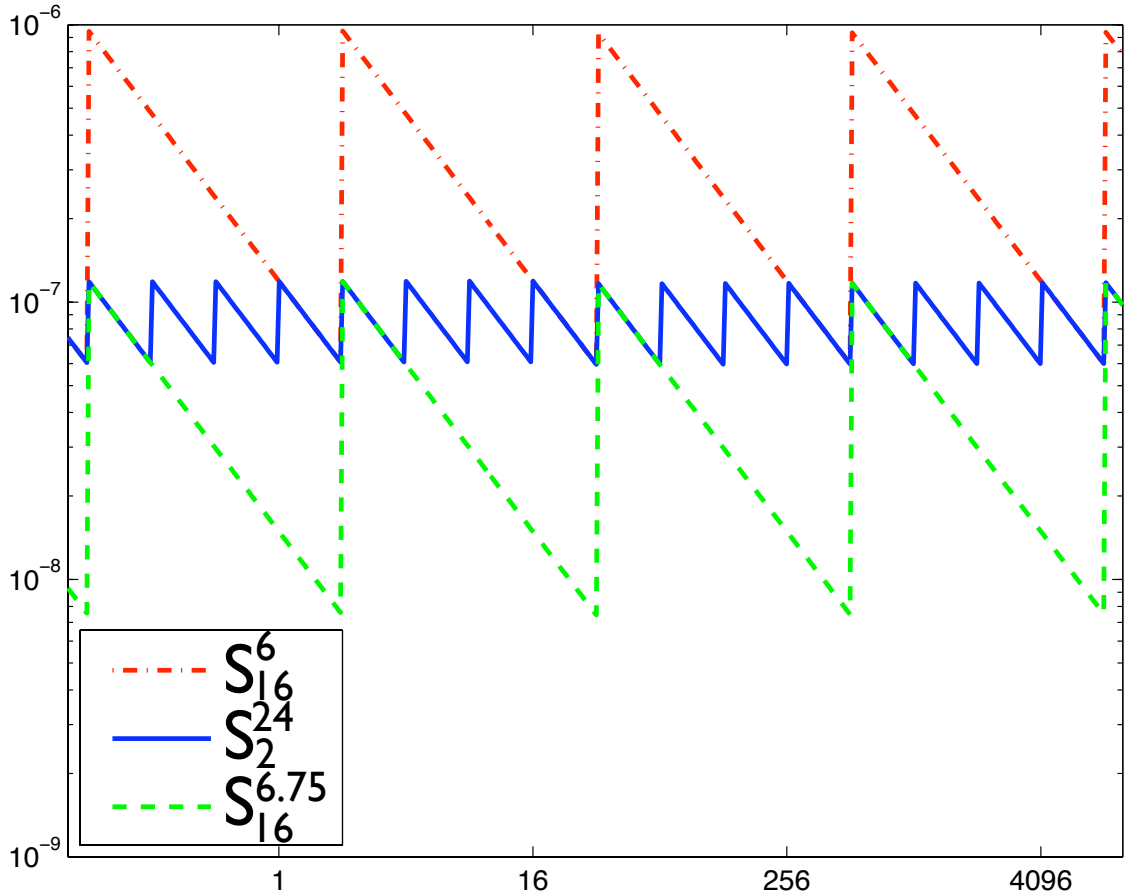

Figure 3.9: Gap Functions for Radix 2 and Radix 16 Representations 
achieve much better average numerical accuracy than fixed point. The fixed-point representation has a much larger gap function, and hence lower precision, for the majority of its range. This occurs since the magnitude information of a fixed-point number is contained in leading zero bits, which reduce numerical precision. Only when representing numbers relatively close to the maximum representable number does fixed-point achieve higher accuracy than a floating-point representation with the same overall width. Examining the gap functions for fixed-point and floating-point representations shows that not only can floating-point numbers represent a huge dynamic range compared to fixed-point, they do so with higher numerical accuracy on average.

This observation can be counterintuitive, since floating-point representations have to carry around an exponent value, which reduces the number of bits which can be used for numerical precision compared to fixed-point representations. However, not all bits are numerically equal - the leading zero bits necessary for representing smaller numbers in fixed-point representations are not numerically significant. Floating-point representations achieve higher accuracy because of normalization, which ensures that mantissa bits are not wasted with leading zeros, and therefore greatly improves numerical accuracy.

Figure 3.9 shows gap functions for 3 floating-point representations, including IEEE single precision $\left(S_{2}^{24}\right)$, as well as two hexadecimal representations. Because a radix $2^{\nu}$ representation allows up to $\nu-1$ leading zeros in the mantissa, its numerical accuracy suffers at equal mantissa width, as seen by the gap functions of $S_{2}^{24}$ and $S_{16}^{6}$, which both have 24 bits of mantissa.

Adding $\nu-1$ bits to a higher radix representation equalizes the worst case gap, or equivalently, the worst case accuracy, as shown by the maxima of the gap functions for $S_{2}^{24}$ and $S_{16}^{6.75}$ (Radix 16, 27 bit mantissa). However, the gap function also shows that $S_{16}^{6.75}$ represents the real numbers on average much more densely than $S_{2}^{24}$. 


\subsection{Rounding Procedures}

Moving to a higher radix also affects rounding procedures. As mentioned earlier, there are several different types of rounding defined in the IEEE specification - the default and most numerically accurate is unbiased rounding to nearest even, and since it is also the most complicated rounding procedure, we will focus on how this mode must be implemented to preserve its numerical properties with higher radix representations.

We will consider addition first, since its rounding procedure is the most complicated. Before the add takes place, the radix points of the two operands must be aligned. This is accomplished by shifting the mantissa of the smaller operand to the right as dictated by the difference in their exponents. When shifting the mantissa to the right, significant bits will be shifted away into oblivion. However, we stated earlier that we want to produce the same result as if those bits had not been lost. In order to do this, in radix 2 addition there are three extra bits which are added to the least significant end of the smaller addend, which are usually called the Guard, Round and Sticky bits [41]. Since the function of these bits can be confusing, their function will be explained for each of the possible scenarios that may occur during a radix 2 addition.

The first case is effective subtraction, exponent difference $>1$. If the difference of the exponents of the two operands is greater than 1, there will be at most one leading zero in the result of the add operation. This means that the normalization step will require a left shift of at most one bit. This left shift requires shifting in a bit, which must be the most significant bit which was lost during alignment in order to preserve all possible precision. This bit is called the Guard bit, since it guards against loss of precision due to alignment. The next bit which was shifted out during alignment, which is called the Round bit, is needed to determine whether we round up or down. Finally, for unbiased rounding, all other bits which were aligned away are logically ored together to form the Sticky bit. The Sticky bit also generates 
the borrow that would have been caused by the nonzero bits that were shifted away during alignment.

The next scenario is effective subtraction, when the exponent difference $\leq 1$. If the difference of the exponents of the two operands is 0 or 1 , there may be many leading zeros in the result, which will cause a massive left shift during normalization. It is fortunate that this case only happens when the difference of the exponents is 0 or 1 , since this means that only 0 or 1 bits has been shifted out during alignment, and therefore, 1 Guard bit is sufficient to preserve all the information from the two operands. In this case, the Round bit and the Sticky bit must be zero, since nothing was shifted into them.

Finally, when performing an effective addition, no leading zeros are produced, therefore no left shift will occur. Only one bit must be saved from alignment, in order to determine whether to round up or down. For unbiased rounding, it is also necessary to calculate the sticky bit in order to know if all the other, discarded bits were zero.

For higher radix addition, the Guard bit must be turned into a Guard digit in order for it to retain all the significant bits that may be shifted out during alignment, and then shifted back in during normalization. The function of the Round and Sticky bits doesn't change, and so they remain as in radix 2.

To accomplish this, instead of 3 round bits needed for radix 2, we now have $\nu+2$ round bits. This rounding procedure is included in the higher radix adder presented in this work.

For multiplication, since there is no equivalent of the alignment phase of addition which shifts away significant bits of one of the operands, there is no need for a guard digit. Unbiased rounding requires one round bit to determine whether the result should be rounded up or down, and the sticky bit to signal whether all other bits of the result are 0 . The rounding procedures remain as they are in radix 2 operations. 


\subsection{Summary}

In this chapter, a family of higher radix representations designed for FPGAbased floating-point computation has been presented. The representations match or exceed the numerical performance of IEEE standard formats in all dimensions: they have equal worst case numerical accuracy, better average case numerical accuracy, and larger dynamic range than the standard formats. They are designed specifically for numerical compatibility with the IEEE representations: every representable number in an IEEE floating-point format is exactly representable in its corresponding higher radix representation, and the radix point position has been carefully chosen to minimize the work necessary to convert from IEEE representations to higher radix representations, and vice versa. The use of flag bits to minimize unnecessary encoding and decoding of exceptional numbers has been outlined. Rounding algorithms for addition and multiplication have been presented which preserve the numerical properties of the IEEE round to nearest even procedure. These higher radix representations take the unique strengths and weaknesses of modern FPGAs into consideration, and are designed to minimize FPGA implementation costs. 


\section{Chapter 4}

\section{Implementation}

Using the parameterization capability of JHDL [42, we have implemented an unpipelined adder and multiplier which are parameterizeable in both bitwidth and radix, as well as conversion circuitry between radix 2 and radix 16 . We also implemented pipelined Single Precision adders and multipliers in radix 2 and radix 16. The operators use the family of higher radix representations outlined earlier in this work.

The divider and square root units have not been created, neither has an analysis of the impact of higher radix on their operation been attempted. Although this is important, it has been left to future work.

All experiments were placed and routed on a Xilinx Virtex-II 6000, speed grade 6 , with embedded multiplier stepping 1 . No hand or relative placement was used. In fact, the circuits had significantly better time and area characteristics when the relative placement mappings assigned automatically by JHDL were stripped from the EDIF netlist. The implemented operators were forced into a compact, contiguous mapping on the FPGA fabric by assigning their input and output pins such that the circuitry for the operator was placed in a corner of the FPGA. Allowing the place and route tools to implement the operators without restricting the input and output pin locations resulted in placements which scattered the operator across the entire FPGA fabric, resulting in unacceptable routing delays.

We present results for radix 16 and radix 4, since they are easily convertable to radix 2 and are therefore of greatest interest. All circuits implement the round to nearest even rounding procedure, as well as support for denormalized numbers. 
When reference is made to single precision, etc., the high radix circuits have equal worst case accuracy and equal dynamic range as their IEEE radix 2 counterparts, i.e. they use the formats described above, including the extension of the mantissa by $\nu-1$ bits, and the contraction of the exponent by $\left\lfloor\log _{2} \nu\right\rfloor$ bits. Thus, the hexadecimal representation compared against IEEE single precision has 6 bits of exponent and 27 bits of mantissa, while its radix 2 counterpart has 8 bits of exponent and 24 bits of mantissa.

\subsection{Unpipelined Adder}

Our adder implements the canonical single path floating-point adder architecture as outlined [13], 41], and shown in figure 4.1.

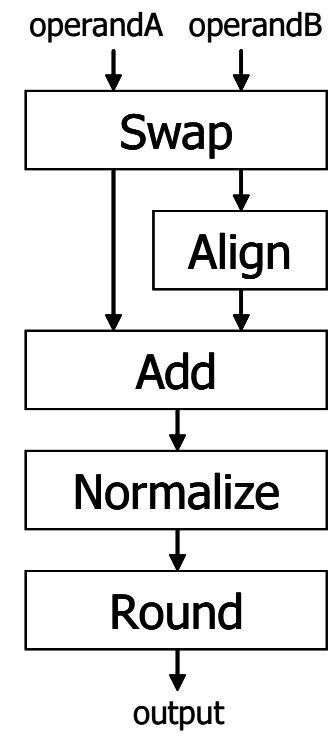

Figure 4.1: Floating-point Adder

The first unit in the adder is the Swap unit, which compares the exponents of the two operands in order to decide which is the largest. The absolute difference of the exponents indicates how much the smaller mantissa should be right shifted in the align 
unit. If two operands have the same exponent value, we do not compare mantissas at this stage to make sure we know exactly which is larger. Some architectures do the mantissa comparison as well, so as to guarantee that in case of subtraction, the result will not be negative. We have chosen to allow the subtraction result to be negative, then invert it if necessary. This unit is not affected by radix.

The Align unit takes care of right shifting the mantissa of the smaller number, generating the correct sticky bit as bits are shifted off the least significant end. To comply with our rounding procedure, $\nu+1$ bits are added to the least significant end of the mantissa in order to catch any significant bits which may be shifted into the guard digit and round bit. The shifter itself is a logarithmic shifter constructed of 2:1 muxes. The complexity of this unit is significantly reduced by higher radix representations.

The Add unit adds the two mantissas together, ensuring that the result is positive, and detecting overflow. This unit is not affected by radix.

The Normalize unit then shifts away any leading zero bits which may have been created during the add operation. It uses a priority encoder and logarithmic shifter as mentioned earlier. In order to support denormalized numbers, the normalize unit will not shift away all the leading zero bits if doing so would cause the resultant exponent to be negative, for a higher radix number, and less than 1 for a radix 2 number. If overflow occurred at add, the number is right shifted one digit and the sticky bit updated. The Normalize unit is significantly reduced by higher radix representations.

The Round unit then computes whether or not the mantissa should be incremented according to the round to nearest even procedure. Incrementing the mantissa can lead to mantissa overflow, which then may result in another right shift. The exponent is equal to the exponent value of the maximum operand, adjusted according to the shifts which were performed after the add operation. Every right shift of one digit results in the exponent being incremented, and complimentarily, every left shift results in decrementing the exponent. The round unit also updates the new flag bits for the result. The round unit is not affected by radix. 


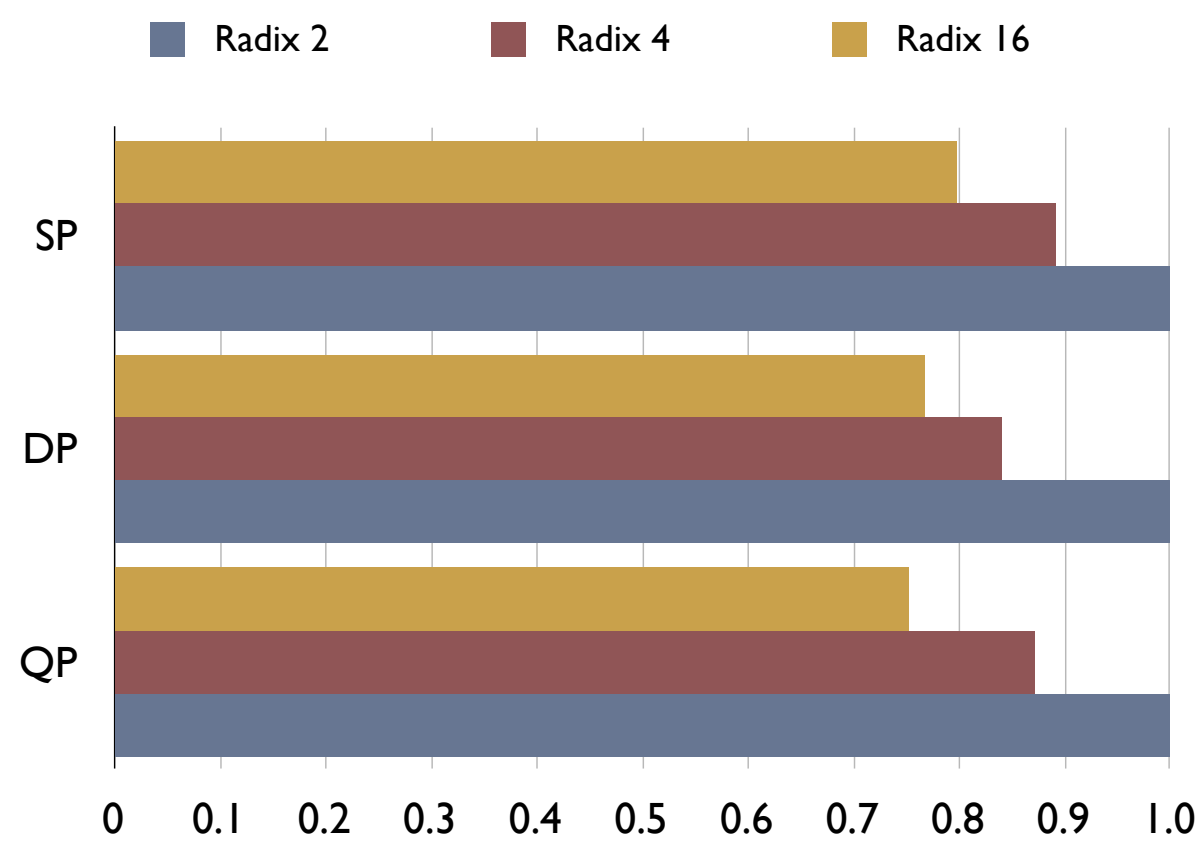

Figure 4.2: Area for Unpipelined Adders Normalized to Radix 2 Adder

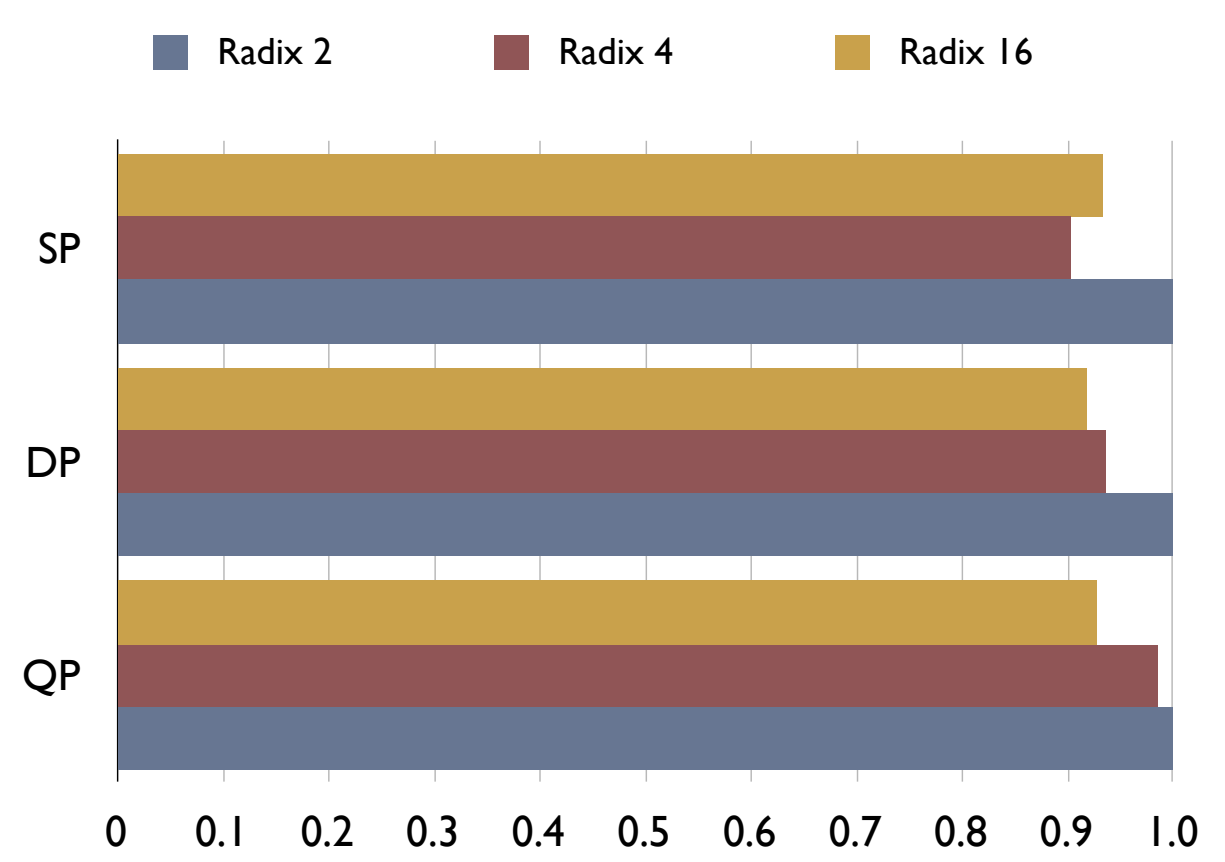

Figure 4.3: Latency for Unpipelined Adders Normalized to Radix 2 Adder 
Table 4.1: Unpipelined Adder Area Comparison

\begin{tabular}{|c|c|c|c|}
\hline Precision & Radix 2 & Radix 4 & Radix 16 \\
\hline Single & 521 LUTs & 465 LUTs & 416 LUTs \\
\hline Double & 1176 LUTs & 989 LUTs & 903 LUTs \\
\hline Quadruple & 2581 LUTs & 2251 LUTs & 1945 LUTs \\
\hline
\end{tabular}

Table 4.1 shows radix 4 gaining from $11 \%$ at single precision to $13 \%$ at quadruple precision over the standard binary adder, while radix 16 benefits from $20 \%$ at single precision to $25 \%$ at quadruple precision. Figure 4.2 shows adder areas normalized to the radix 2 adder.

Table 4.2: Unpipelined Adder Timing Comparison

\begin{tabular}{|c|c|c|c|}
\hline Precision & Radix 2 & Radix 4 & Radix 16 \\
\hline Single & $51.5 \mathrm{~ns}$ & $46.6 \mathrm{~ns}$ & $48.1 \mathrm{~ns}$ \\
\hline Double & $66.9 \mathrm{~ns}$ & $62.7 \mathrm{~ns}$ & $61.4 \mathrm{~ns}$ \\
\hline Quadruple & $89.4 \mathrm{~ns}$ & $88.2 \mathrm{~ns}$ & $83.0 \mathrm{~ns}$ \\
\hline
\end{tabular}

Table 4.2 illustrates that the combinatorial critical path through high-radix adders is reduced slightly, around $5 \%$ for radix 4 and $7 \%$ for radix 16 . Figure 4.3 shows adder latencies normalized to the radix 2 adder.

The benefits we have seen using radix 16 are greater than those observed in [39] for several reasons. Firstly, shifters are relatively cheaper in VLSI technology than in FPGA fabric, since they can use more efficient transistor level structures specifically designed for shifting. This reduces the impact of minimizing the shifters, in contrast to FPGAs, on which shifters are expensive. Secondly, 39] examines the benefit of hexadecimal floating-point representations at very small word sizes. As can be seen in table 4.1 , the benefit from higher radix representations increases with word size. 


\subsection{Unpipelined Multiplication}

Our multiplier uses the single-path architecture outlined in [1], and supports denormalized numbers. The multiplier makes use of embedded block multipliers for the mantissa multiplication. Figure 4.4 shows the overall block diagram of the multiplier.

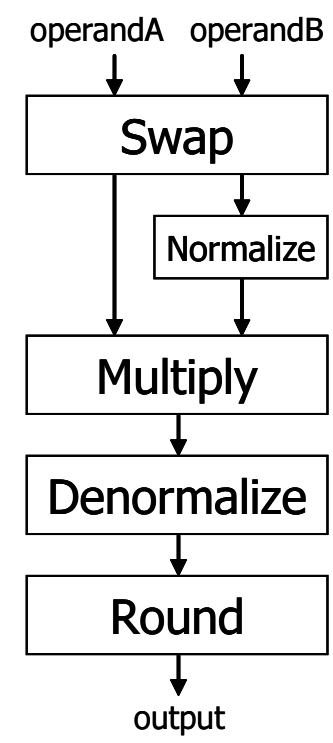

Figure 4.4: Floating-point Multiplier

The multiplier architecture is different from the majority of floating-point multiplier architectures, such as those presented in [41], because it supports denormalized numbers. Since multiplying a denormalized number by a normalized number may result in a denormalized or a normalized number, and since multiplying two small normalized numbers may result in a denormalized number, all the corner cases must be carefully thought through. As in [1, we use a swap unit at the beginning of the multiplier. The task of the multiplier swap unit is to identify which argument is a denormalized number, for the case when a normalized number is being multiplied by a denormalized number. If two denormalized numbers are being multiplied together, 
the result will end up being flushed to zero. This occurs because the exponent value of the result of multiplying two negative numbers is guaranteed to be so negative that the entire result will be of less magnitude than $1 / 2$ of the smallest representable denormalized number, which results in a zero result. Because of this fact, the operation does not need to take into consideration the case where both inputs are denormalized numbers.

If a denormalized operand is found, we normalize it.This eliminates the need for a large priority encoder at the output of the mantissa multiplier, since we then know where the leading non-zero digit is going to be.

The mantissa multiplier stage is made from block multipliers and an adder network which stitches the block multipliers together to form a full mantissa width multiplier.

The Denormalize stage is present for the case when the result should be a denormalized number. Since the result of the multiplication will be more or less normalized, we may need to introduce leading zero digits. The denormalize stage also takes care of normalizing the result completely. Interval arithmetic reminds us that the result of a normalized radix 2 multiplication with both mantissas in the range $[1,2)$ will have a mantissa in the range $[1,4)$, while the result of a normalized higher radix multiplication with both mantissas in the range $\left[\frac{2}{\beta}, 2\right)$ will have a mantissa in the range $\left[\frac{4}{\beta^{2}}, 4\right)$. Thus, the radix 2 multiplier has 2 ranges to select between to produce a normalized result: $[1,2)$ and $[2,4)$, while the higher radix multiplier has 3 ranges to choose from: $\left[\frac{4}{\beta^{2}}, \frac{2}{\beta}\right),\left[\frac{2}{\beta}, 2\right)$, and $[2,4)$. This selection is done in the Denormalize stage.

Finally, we have the Round stage, which implements the round to nearest even algorithm to increment the mantissa, taking care of all the corner cases with mantissa overflow, etc., and generating new flag bits for the output. The exponent of the result is equal to the sum of the two exponents of the operands, minus the bias, and then adjusted by all the shifting which took place to get the number properly normalized or denormalized. 


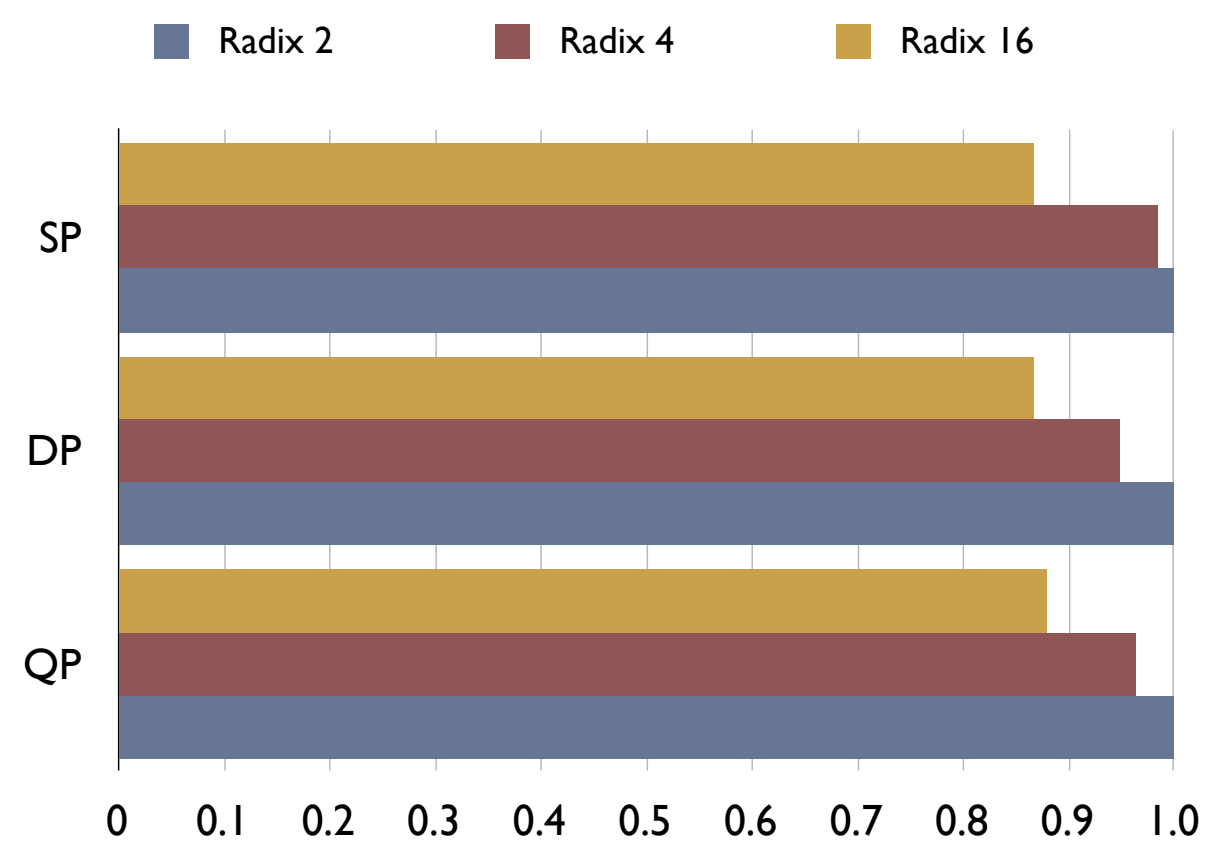

Figure 4.5: Area for Unpipelined Multipliers Normalized to Radix 2 Multiplier

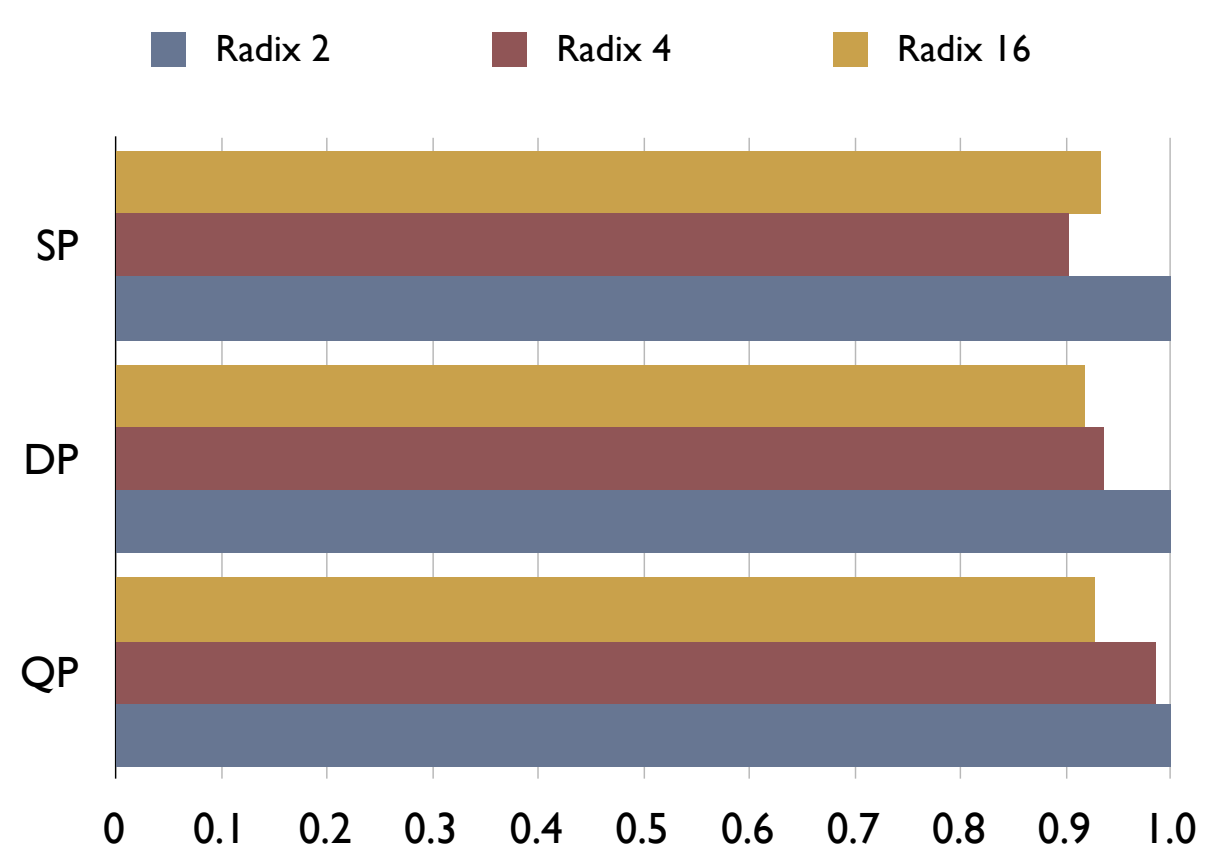

Figure 4.6: Latency for Unpipelined Multipliers Normalized to Radix 2 Multiplier 
Table 4.3: Unpipelined Multiplier Area Comparison

\begin{tabular}{|c|c|c|c|}
\hline Precision & Radix 2 & Radix 4 & Radix 16 \\
\hline Single & 452 LUTs, 4 Mults & 445 LUTs, 4 Mults & 392 LUTs, 4 Mults \\
\hline Double & 1312 LUTs, 16 Mults & 1245 LUTs, 16 Mults & 1139 LUTs, 16 Mults \\
\hline Quadruple & 3559 LUTs, 49 Mults & 3431 LUTs, 49 Mults & 3130 LUTs, 49 Mults \\
\hline
\end{tabular}

Table 4.3 shows that radix 4 multipliers are slightly smaller than their radix 2 counterparts, while radix 16 multipliers are around $12 \%$ smaller. Higher radix operators used exactly the same number of block multipliers as the binary multiplier.

Multipliers which support denormalized numbers must have both a normalizing and a denormalizing shifter, the size of which are reduced by high-radix representations. This results in the area benefit we have observed - if our multiplier did not support denormalized numbers, we would see a small area penalty rather than a savings, due to the added mux and slightly enlarged mantissa multiplier. However, FPGAs see a smaller penalty from the mantissa extension than ASIC implementations because of the discrete area scaling behavior of multipliers constructed from smaller block multipliers. Thus, block multipliers and support for denormalized numbers explain why we observe an area benefit, as opposed to the area penalty seen by [39].

Table 4.4: Multiplier Timing Comparison

\begin{tabular}{|c|c|c|c|}
\hline Precision & Radix 2 & Radix 4 & Radix 16 \\
\hline Single & $49.0 \mathrm{~ns}$ & $56.3 \mathrm{~ns}$ & $52.6 \mathrm{~ns}$ \\
\hline Double & $73.5 \mathrm{~ns}$ & $86.9 \mathrm{~ns}$ & $74.7 \mathrm{~ns}$ \\
\hline Quadruple & $108.0 \mathrm{~ns}$ & $122.2 \mathrm{~ns}$ & $116.5 \mathrm{~ns}$ \\
\hline
\end{tabular}


The combinatorial critical path through our high radix multipliers was increased from $2-8 \%$ for the hexadecimal multiplier, and somewhat more for the radix 4 multiplier. This is primarily due to the enlarged mantissa multiplier.

\subsection{Converter Hardware}

As explained earlier, the hardware necessary to convert a radix 2 representation to a radix $\beta$ representation is simplified when $\beta=2^{2^{k}}$. Of radices that satisfy this condition, radix 16 seems to be optimal, since it yields more hardware savings than radix 4, yet doesn't require the floating-point word size to be lengthened excessively to compensate for reduced accuracy, as do large radices such as 256 .

Since a hexadecimal floating-point representation is 2 bits longer than its corresponding binary counterpart, some applications will require keeping the datapath externally radix 2 but internally radix 16, stationing converters at the gateways to the circuit. Although converter circuitry may be necessitated by higher radix representations, it is worth noting that FPGA-based floating-point datapaths gain performance by keeping data on chip as much as possible, especially since FPGAs are very pinlimited compared with the parallelism that can be accomodated internally. These two facts combined support the assertion that relatively few of these converters should be needed, and the overall system cost should be reduced by using a higher radix representation.

We chose the implied binary point placement to simplify conversion between standard radix 2 and radix 16. Because of this choice, conversion from radix 2 to radix 16 requires only a shifter which shifts the mantissa $0-3$ places to the right, as determined by the bottom 2 bits of the exponent, which are then discarded to form the radix 16 exponent. A small bit of logic is required to handle exponent corner cases. No rounding is necessary, since no significant bits are lost in the conversion.

The conversion from radix 16 back to radix 2 requires a shifter to shift the mantissa 0-3 places to the left, eliminating the leading zeros. Since the radix 16 format can represent more numbers than the radix 2 format, a round operation is required to choose the closest representable radix 2 number, and some logic must be 
included for exponent corner cases. In order to avoid instantiating a rounder in this converter, we integrate the converter into the normalization and rounding steps of the arithmetic operators, making hybrid radix operators which accept hexadecimal numbers and output binary, IEEE results.

Table 4.5: Converter Circuitry Area

\begin{tabular}{|c|c|c|}
\hline Precision & Radix $2 \rightarrow$ Radix 16 & Radix $16 \rightarrow$ Radix 2 \\
\hline Single & 50 LUTs & 104 LUTs \\
\hline Double & 108 LUTs & 229 LUTs \\
\hline Quadruple & 229 LUTs & 484 LUTs \\
\hline
\end{tabular}

The cost of these converters is reasonable: in the worst case scenario with a datapath comprised of a radix $2 \rightarrow$ radix 16 converter, a single radix 16 adder, and a radix $16 \rightarrow$ radix 2 converter, the aggregate cost is between from $2-9 \%$ more than the cost of a single radix 2 adder. Since FPGAs gain their performance by performing multiple calculations and limiting I/O, few of these converters should be needed compared to the number of arithmetic operators in the datapath. Thus, using hexadecimal floating-point internally and binary floating point externally should reduce overall system cost, despite the use of converter circuitry.

\subsection{Pipelined Operators}

Using the same architecture as the unpipelined operators outlined above, pipelined single precision adders and multipliers were constructed for radix 2 and radix 16 formats.

Figure 4.7 shows the area of the pipelined operators in slices. As predicted earlier, pipelining did not change the area improvements significantly over the unpipelined versions. For single precision, the adder is still $20 \%$ smaller, and the multiplier is still $10 \%$ smaller. 


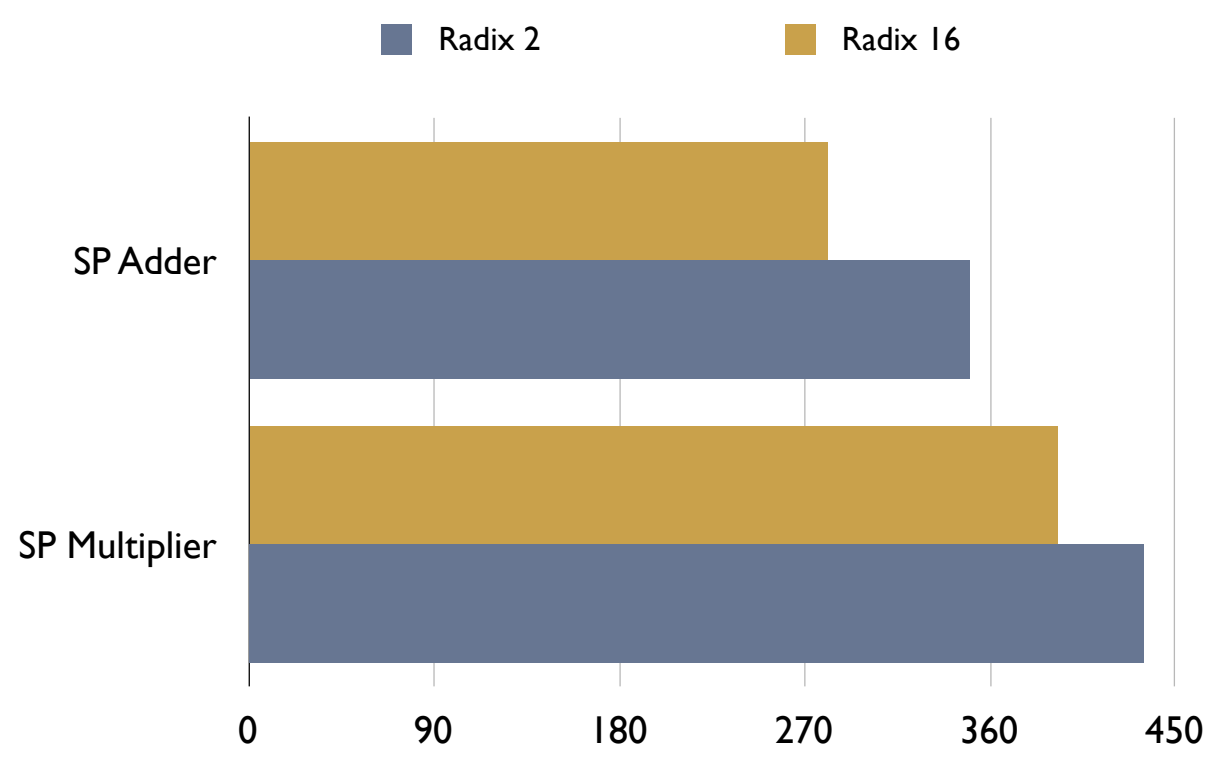

Figure 4.7: Area of Pipelined Operators in Slices

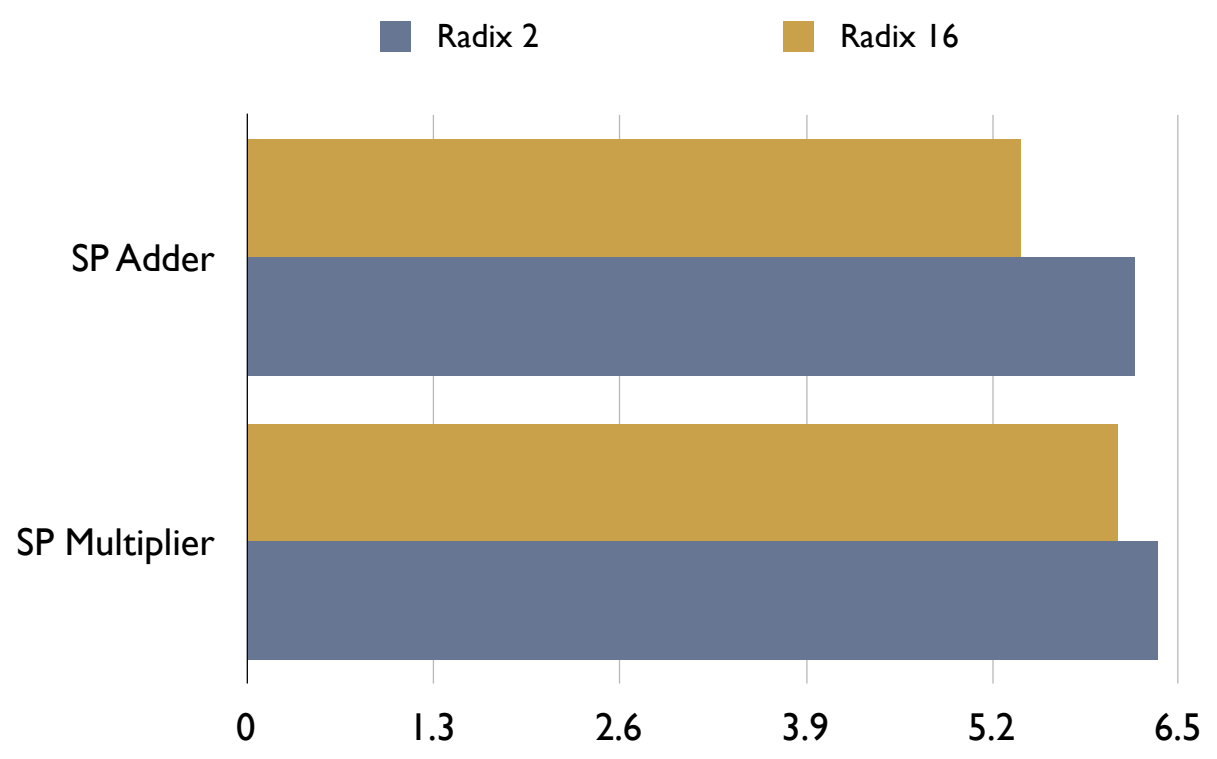

Figure 4.8: Period of Pipelined Operators in Nanoseconds 
Figure 4.8 shows the clock period which was achieved after pipelining. The pipelined adders both have latency of 9 cycles, while the multipliers have latency of 10 cycles. The radix 2 adder has a relatively large cycle time penalty. The critical path of the radix 2 adder was found to be the priority encoder, which is significantly larger in a radix 2 adder. If the radix 2 adder were pipelined to achieve the same clock speed as the radix 16 adder, area would increase. The radix 16 multiplier achieved around $5 \%$ faster period, however, the stochastic nature of FPGA place and route algorithms makes one hesitant to pronounce this a meaningful result.

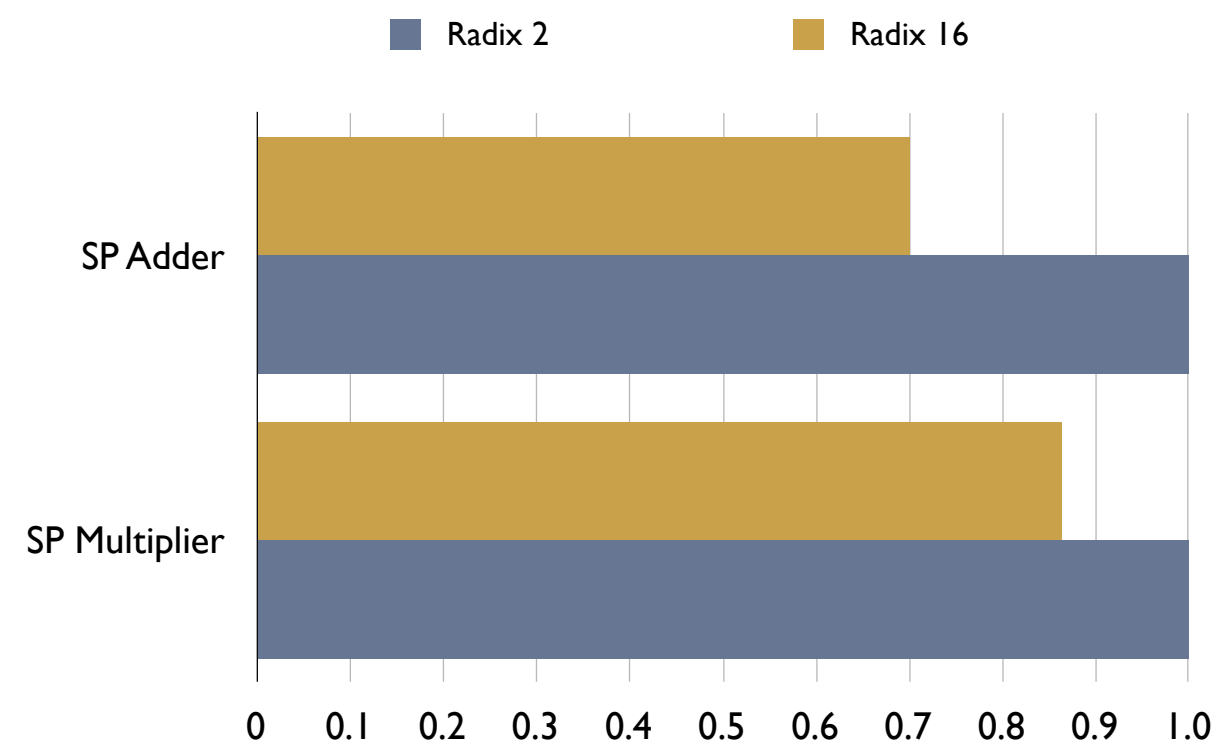

Figure 4.9: Pipelined Area Time Products Normalized to Radix 2

Finally, figure 4.9 shows the normalized area time products for radix 2 and radix 16 adders and multipliers. The radix 16 adder achieves a 30\% area-time reduction, while the radix 16 multiplier achieves a $13 \%$ area-time reduction. 


\subsection{Floating-Point Unit Building Blocks}

The shifters and priority encoders are greatly affected by the choice of radix, and are responsible for the area and time savings demonstrated above. Examining their scaling behavior as a function of radix is warranted, in order to understand why higher radix representations are more efficient on FPGAs.

\subsubsection{Priority Encoder}

The priority encoder is one of the critical circuits in any floating-point operator, and higher radix representations drastically reduce its area and latency cost. Its function is to find the leading non-zero digit, which is crucial information for a normalized floating-point format. In this work, we implemented the priority encoder using fast carry logic, which drastically reduces the latency of the priority encoding operation compared to the naive LUT-based implementation. The topology presented here is modified from [33].

Figure 4.10 illustrates a 25 bit, radix 2 priority encoder. In floating-point operators, the priority encoders used are sized to match the mantissa width. For the adder, the priority encoder must also include the guard digit, which is why the priority encoder in Figure 4.10 is 25 bits long. Its radix 16 counterpart is 31 bits long, and it is shown in Figure 4.11 .

Obviously, the radix 16 priority encoder is than the corresponding radix 2 priority encoder, despite the fact that it operates on a longer word.

\subsubsection{Normalizing and Aligning Shifters}

The bulk of the hardware savings comes from reducing the size of the normalizing and aligning shifters. Since a radix $2^{\nu}$ shifter only has to shift to within $\nu$ bits, the amount of shifting which must be performed is reduced significantly.

Figure 4.12 illustrates this benefit for the normalizing shifter of the single precision radix 16 adder. The shifter must shift 0 to 7 radix-16 digits, requiring 3 stages of 2 input muxes. The corresponding radix 2 shifter, shown in figure 4.13 


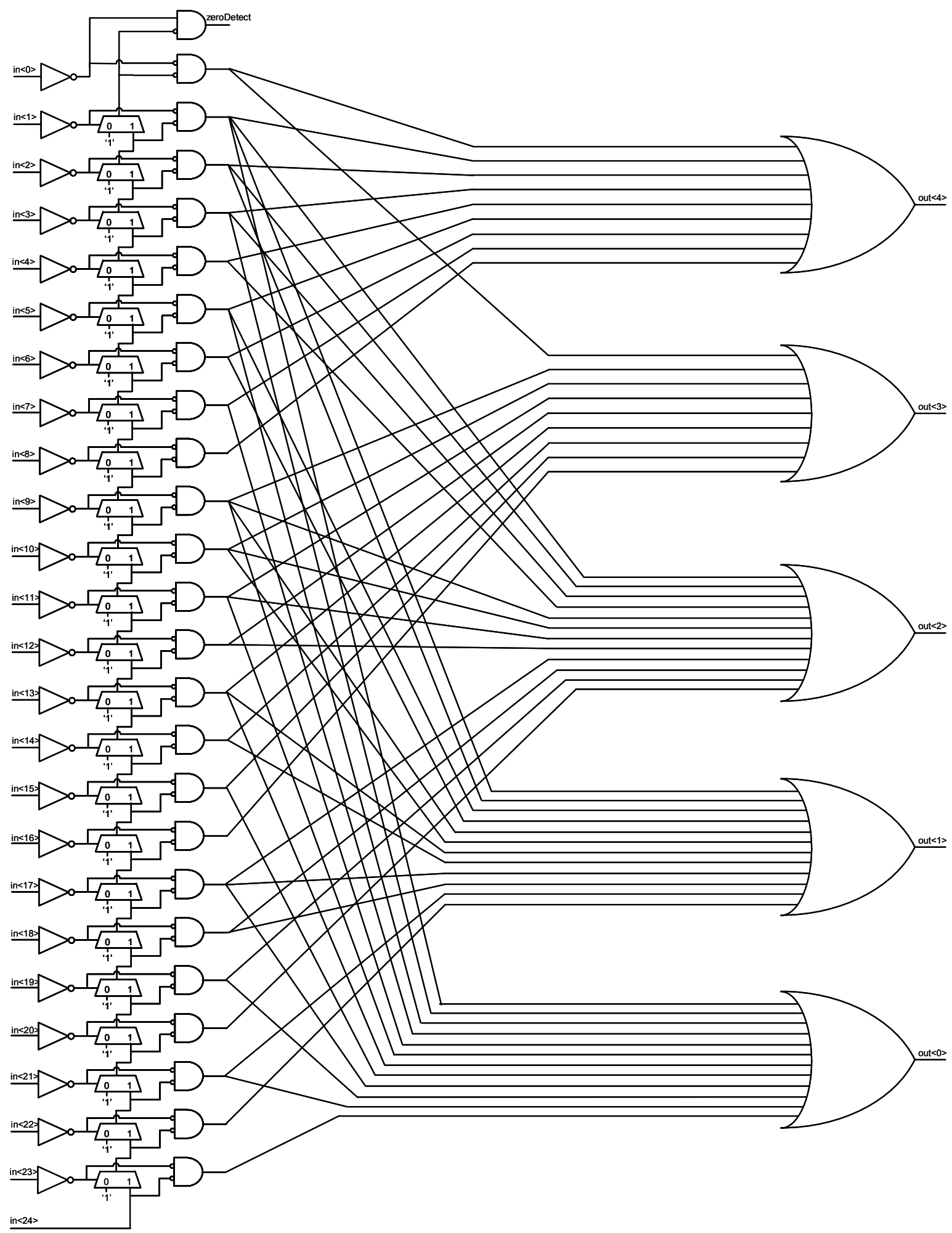

Figure 4.10: Radix 2, 25 Bit Priority Encoder 


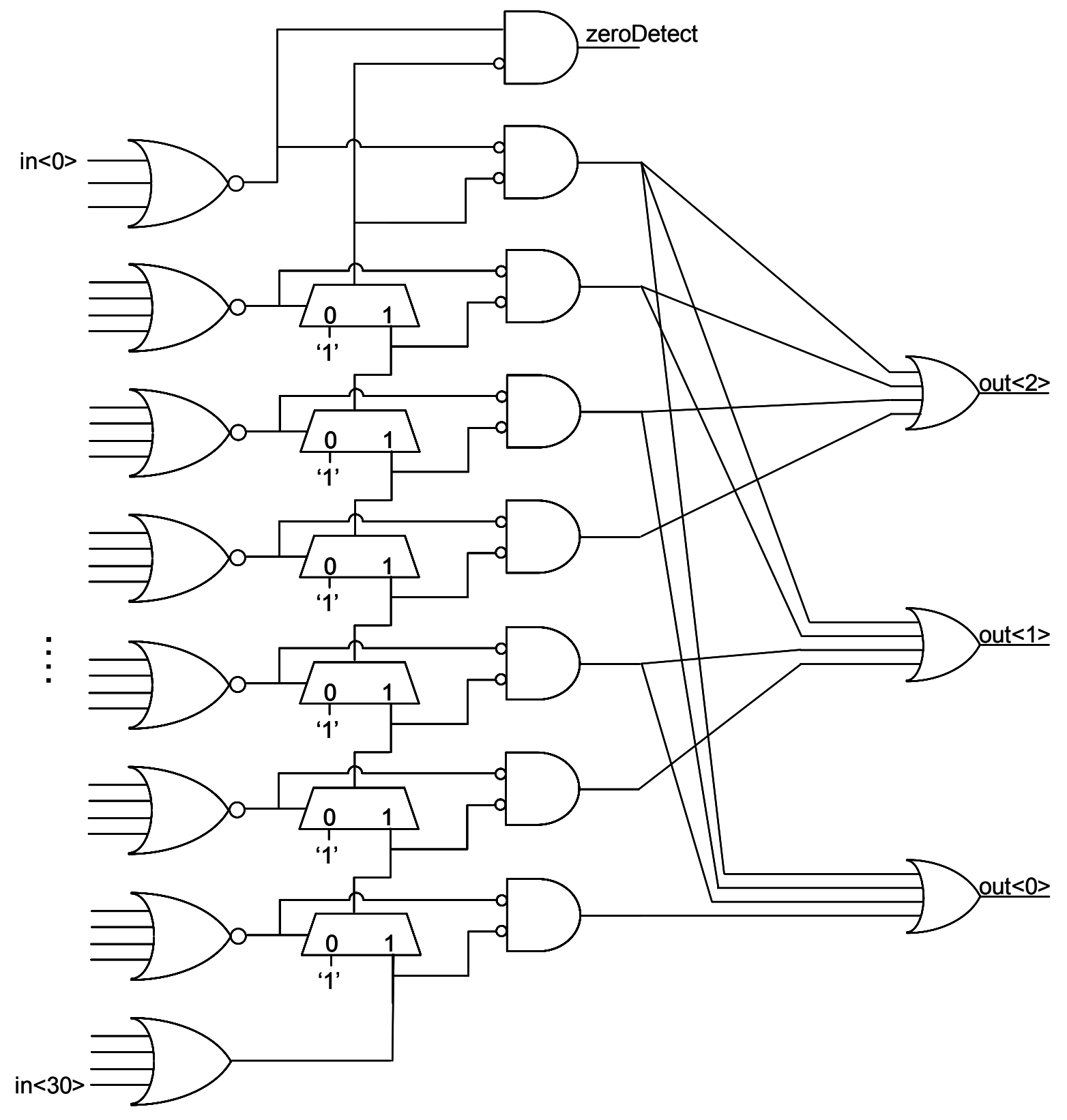

Figure 4.11: Radix 16, 31 Bit Priority Encoder 


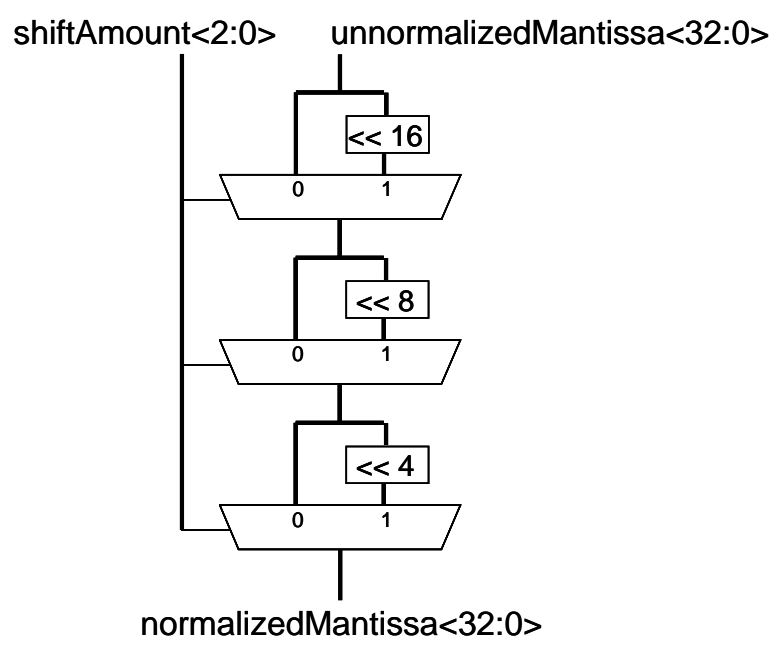

Figure 4.12: Radix 16 Normalizing Shifter

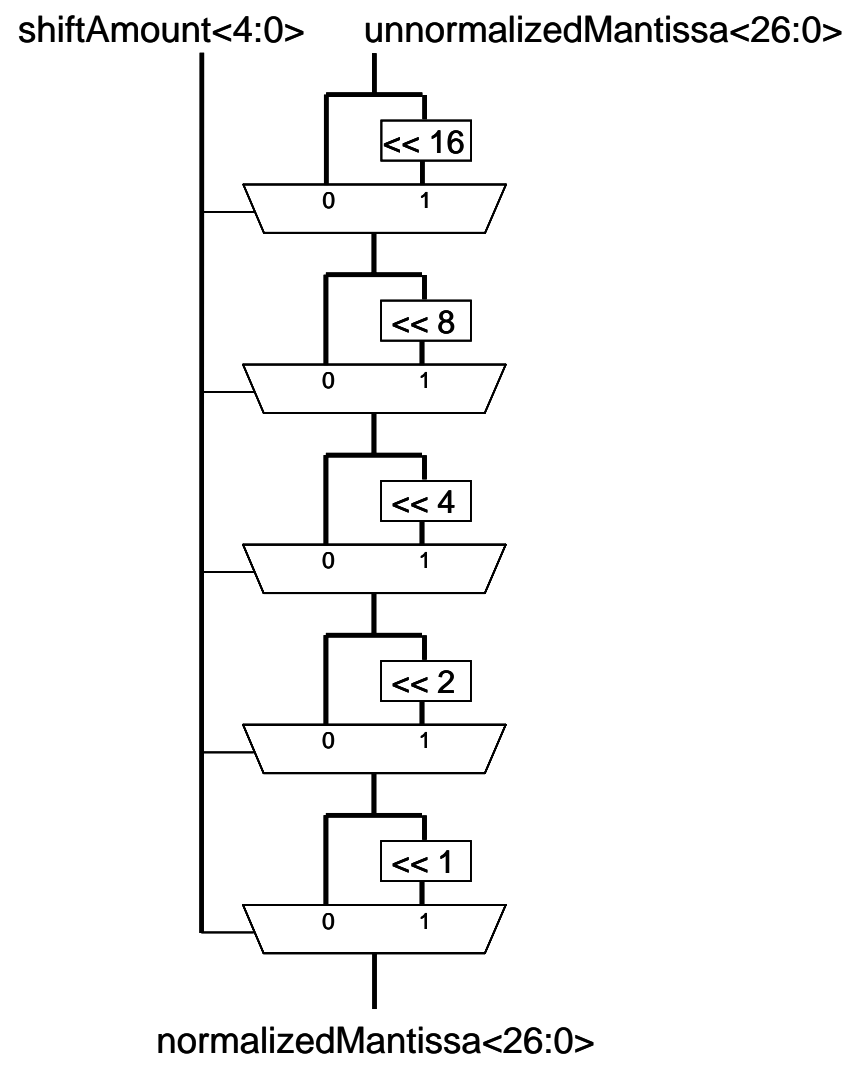

Figure 4.13: Radix 2 Normalizing Shifter 
must shift 0 to 24 bits, requiring 5 stages of 2 input muxes. Although some have claimed that making the shifters out of 4 input muxes reduces the latency of the shifter by taking advantage of the fast 4 input mux function provided by modern FPGAs (the F5MUX in Xilinx Virtex, for example) [33, we found that the increased routing congestion caused by 4 input muxes caused a considerable ( $30 \%$ ) frequency reduction for our pipelined operators. Since these shifters occupy a relatively large area, reducing their cost creates most of the area benefit of high radix multipliers and adders.

\subsection{Future Work}

We have not examined the impact of higher radix representations on divider or square-root circuitry.

We expect high radix representations to reduce power consumption similar to or slightly better than they reduce area, although this is as of yet unproven. Choosing a higher radix representation may thus be another chance to lower power consumption. Future work should explore these questions on pipelined versions of our higher radix operators. 


\section{Chapter 5}

\section{Conclusions}

This thesis reexamines the choice of floating-point radix for FPGA-based floating-point datapaths. The established consensus that radix 2 floating-point representations are optimal depends on the assumption that numerical accuracy per bit is the most important criterion by which to choose a floating-point representation. If the criterion is changed to reflect implementation efficiency on FPGAs, higher radix representations, particularly radix 16 , are more attractive. Choosing a higher radix representation can yield implementations with better numerical accuracy, while still reducing area cost. Radix 16 is a particularly good choice, since it provides good area savings, and converters to and from radix 2 are simplified. Designs that are heavily constrained by memory interfaces can either sacrifice some accuracy to fit the representation within a convenient number of bits, or they can use converters at the gateways to the floating-point datapath.

High radix approaches may not be optimal for designs with much I/O and little computation, for designs using very small, non-standard representations, or for

designs with many multipliers and no support for denormalized numbers. For such applications, radix 2 may be the best choice. However, for many designs, higher radix representations can be used to maximize efficiency for floating-point datapaths implemented on FPGAs. Some designers are beginning to push for greater precision than afforded by IEEE double precision, and need support for denormalized numbers [1]. The area savings afforded by higher radix representations, especially when support for denormalized numbers is required, may enable the implementation of such extremely high precision calculations on an FPGA. Since processors with hardware 
quadruple precision units are rare and expensive at present, such calculations must be run in software, making them an even bigger target for FPGA implementation. Calculations requiring less precision can also benefit from higher radix representations, especially if there are proportionally many add operations in the datapath.

Due to the established consensus that binary floating-point is optimal, the choice of floating-point radix has been neglected. The unique traits of FPGAs, such as the high ratio of calculation to I/O, high shifter cost, and embedded block multipliers make higher radix floating-point representations, especially hexadecimal floatingpoint, particularly attractive. Designers of FPGA based custom floating-point datapaths should consider whether a high radix representation would be better suited to their needs. 
Appendix 


\section{Appendix A}

\section{Reducing Embedded Multiplier Usage}

\section{A.1 Justification}

As mentioned earlier, the demand for very high precision floating-point computation is increasing, and FPGAs are especially suited for implementing them. However, implementing the mantissa multiplier for very large mantissas, such as the 113 bit mantissa of quadruple precision, is very expensive, due to the quadratic scaling behavior of integer multipliers.

The introduction of embedded multiplier blocks has significantly reduced the cost of implementing mantissa multipliers. Embedded multipliers included in Xilinx FPGAs are 2's-complement, signed multipliers which accept 18 bit inputs [29]. Altera and Lattice Semiconductor produce FPGAs with blocks of 4 18-bit multipliers which can be stitched together as a 36-bit multiplier with a hard macro [30] 31].

The mantissa multiplication for mantissas larger than can be accepted by a single embedded multiplier is implemented by stitching together embedded multipliers with an adder network. Since mantissa multiplication is an unsigned operation, and the embedded multipliers are signed operators, the sign bit can't be used, effectively making the 18-bit embedded multiplier blocks accept 17 bit inputs and output a 34 bit output. The standard way of stitching together 18-bit signed embedded multipliers involves using $n^{2}$ embedded multipliers, where $n$ is the number of 17-bit digits in the inputs to the multiplier. For example, a 113-bit quadruple precision

mantissa contains $\left\lceil\frac{113}{17}\right\rceil=7$ digits, each 17 bits long, meaning that the multiplier requires $7^{2}=49$ embedded 18-bit multipliers. Since many FPGAs have a limited 


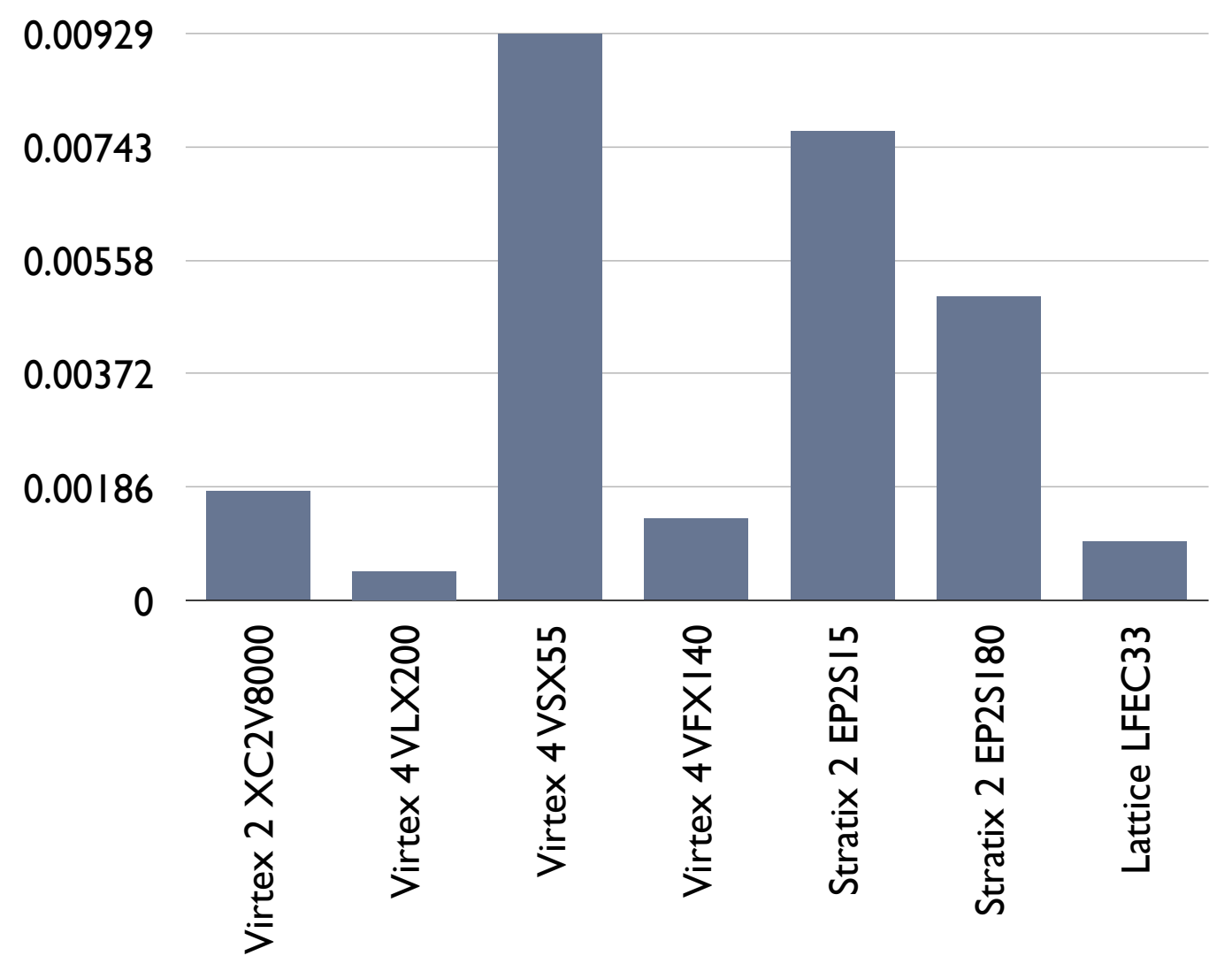

Figure A.1: Number of 18-bit Multipliers/Number of Lookup Tables

number of embedded multipliers, the number of large multipliers which can be fit on chip may be constrained by embedded multiplier availability, and not by general purpose logic resources.

Figure A.1 illustrates the wide variability in proportion of multiplier blocks to lookup table resources. For example, the Xilinx Virtex 4 family alone has a 20x variation from the LX group parts, intended for general logic implementation, to the SX parts, intended for arithmetic applications. Obviously one would try to use a Virtex 4 SX part for implementing heavy-duty arithmetic computations, however, the Virtex 4 SX parts have very limited LUT resources (4x less than the LX family), and so may be LUT limited instead of multiplier limited. Also, sometimes availability constraints require the user to make do with a suboptimal FPGA. 
Such a situation occurred, for example, when a team from Los Alamos National Laboratory implemented a radiative heat-transfer kernel on a Virtex II [37]. In that case, only $20 \%$ of the logic resources of the chip were utilized, but every embedded multiplier block was being used. The disproportionate lack of embedded multipliers on their FPGA severely limited the amount of parallelism, and hence performance, which was accomodated on chip.

In this section, a factorization is presented which allows a multiplier to use asymptotically half the number of block multipliers than are used conventionally. Knuth mentions this factorization for the simple 2 digit case in [15], which allows the multiplication to be accomplished with 3 sub-multiplies instead of 4 . This work generalizes the factorization to inputs of an arbitrary width and examines scaling behavior.

Applying this factorization results in a tradeoff: although the number of submultiplies is decreased by a factor of two, the number of partial product bits to be added to form the product is increased by a factor of 2.5. Since the adder network is much cheaper to implement in FPGA fabric than the multipliers, the resultant multiplier is much more efficient than one implemented completely in general purpose logic. This technique will be of interest to FPGA designers who find themselves multiplier limited, as the Los Alamos team did. For others, the conventional method is more appropriate.

\section{A.2 Factorization}

The standard algorithm for multiplication, using embedded multipliers, is as follows. Let $\nu$ be the number of bits in one unsigned operand input to a block multiplier. Let $n$ be the number of radix $2^{\nu}$ digits in one operand to the multiplication. We can then represent an operand $u$, composed of $n$ digits $u_{k}$ as

$$
u=\sum_{j=0}^{n-1} u_{k}\left(2^{\nu}\right)^{j} .
$$

The product of two numbers $u$ and $v$ is then

$$
u v=\sum_{j=0}^{n-1} u_{k}\left(2^{\nu}\right)^{j} \sum_{k=0}^{n-1} v_{k}\left(2^{\nu}\right)^{k}
$$




$$
=\sum_{j=0}^{n-1} \sum_{k=0}^{n-1} u_{j} v_{k} 2^{\nu(j+k)} .
$$

The key factorization which enables us to reduce the demand for block multipliers is to realize that

$$
\left(u_{j}-u_{k}\right)\left(v_{k}-v_{j}\right)=u_{j} v_{k}+u_{k} v_{j}-u_{j} v_{j}-u_{k} v_{k}
$$

or equivalently,

$$
u_{j} v_{k}+u_{k} v_{j}=\left(u_{j}-u_{k}\right)\left(v_{k}-v_{j}\right)+u_{j} v_{j}+u_{k} v_{k}
$$

This is helpful when $j \neq k$ : we generate the $u_{j} v_{k}$ and $u_{k} v_{j}$ partial products with only one multiplication instead of two. Although the multiplication changes from an unsigned multiplication to a signed multiplication, since the embedded multipliers in FPGA fabrics are signed, this does not increase complexity. The additional partial product terms $u_{j} v_{j}$ and $u_{k} v_{k}$ do not incur any additional multiplies, since those terms must be calculated anyway. Substituting equation A.4 into equation A.2 and rearranging terms,

$$
\begin{aligned}
u v & =\sum_{j=0}^{n-1} u_{j} v_{j} 2^{\nu(2 j)}+\sum_{j=0}^{n-2} \sum_{k=j+1}^{n-1}\left[\left(u_{j}-u_{k}\right)\left(v_{k}-v_{j}\right)+u_{j} v_{j}+u_{k} v_{k}\right] 2^{\nu(j+k)} \\
& =\sum_{j=0}^{n-1} u_{j} v_{j} 2^{\nu(2 j)}+\sum_{j=0}^{n-2} \sum_{k=j+1}^{n-1}\left(u_{j} v_{j}+u_{k} v_{k}\right) 2^{\nu(j+k)}+\sum_{j=0}^{n-2} \sum_{k=j+1}^{n-1}\left[\left(u_{j}-u_{k}\right)\left(v_{k}-v_{j}\right)\right] 2^{\nu(j+k)} \\
& =\sum_{j=0}^{n-1} u_{j} v_{j} \sum_{l=j}^{j+n-1} 2^{\nu l}+\sum_{j=0}^{n-2} \sum_{k=j+1}^{n-1}\left[\left(u_{j}-u_{k}\right)\left(v_{k}-v_{j}\right)\right] 2^{\nu(j+k)} .
\end{aligned}
$$

Equation A.5 is the generalized factorization which allows us to reduce the number of embedded multipliers used to compute a multiplication.

\section{A.3 Architecture}

From equation A.5, it can easily be seen that the number of embedded multiplies needed is

$$
\begin{gathered}
n+\sum_{j=0}^{n-2} n-j-1= \\
n+\frac{n^{2}-n}{2}= \\
\frac{n^{2}+n}{2} .
\end{gathered}
$$


The conventional multiplication algorithm requires $n^{2}$ embedded multipliers, from which it follows that this factorization asymptotically reduces the number of embedded multipliers by a factor of 2 .

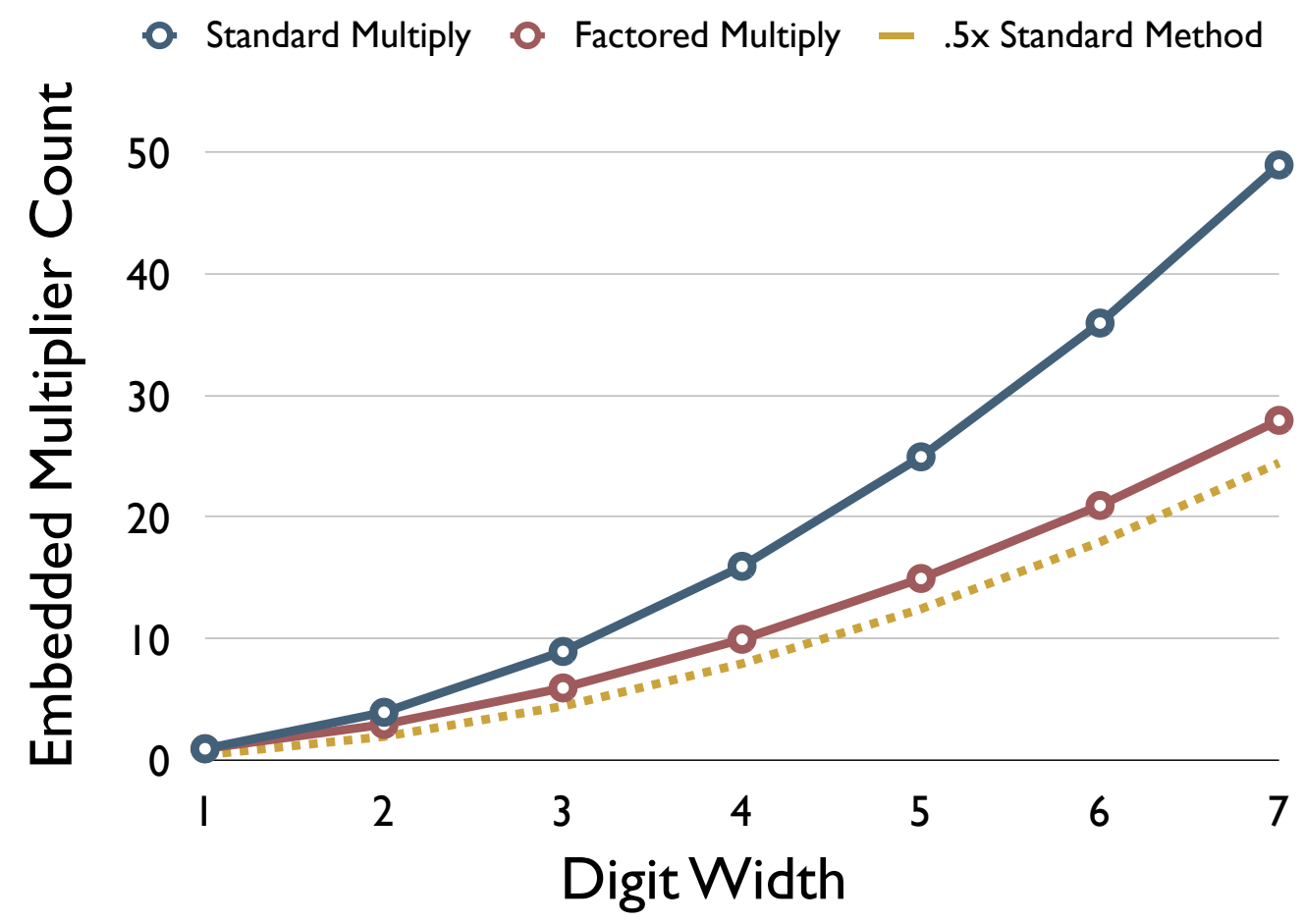

Figure A.2: Number of Block Multipliers Versus Input Digit Width

Figure A.2 shows how many embedded multipliers are required as a function of the number of 17-bit digits in each operand.

As a rough measure of the increase in adder tree complexity which accompanies this factorization, we consider the number of partial product bits which must be added to create the product.

For the conventional algorithm, there are $n^{2}$ partial products, each of which are $2 \nu$ bits long, for a total of $2 \nu n^{2}$ bits of partial product. 


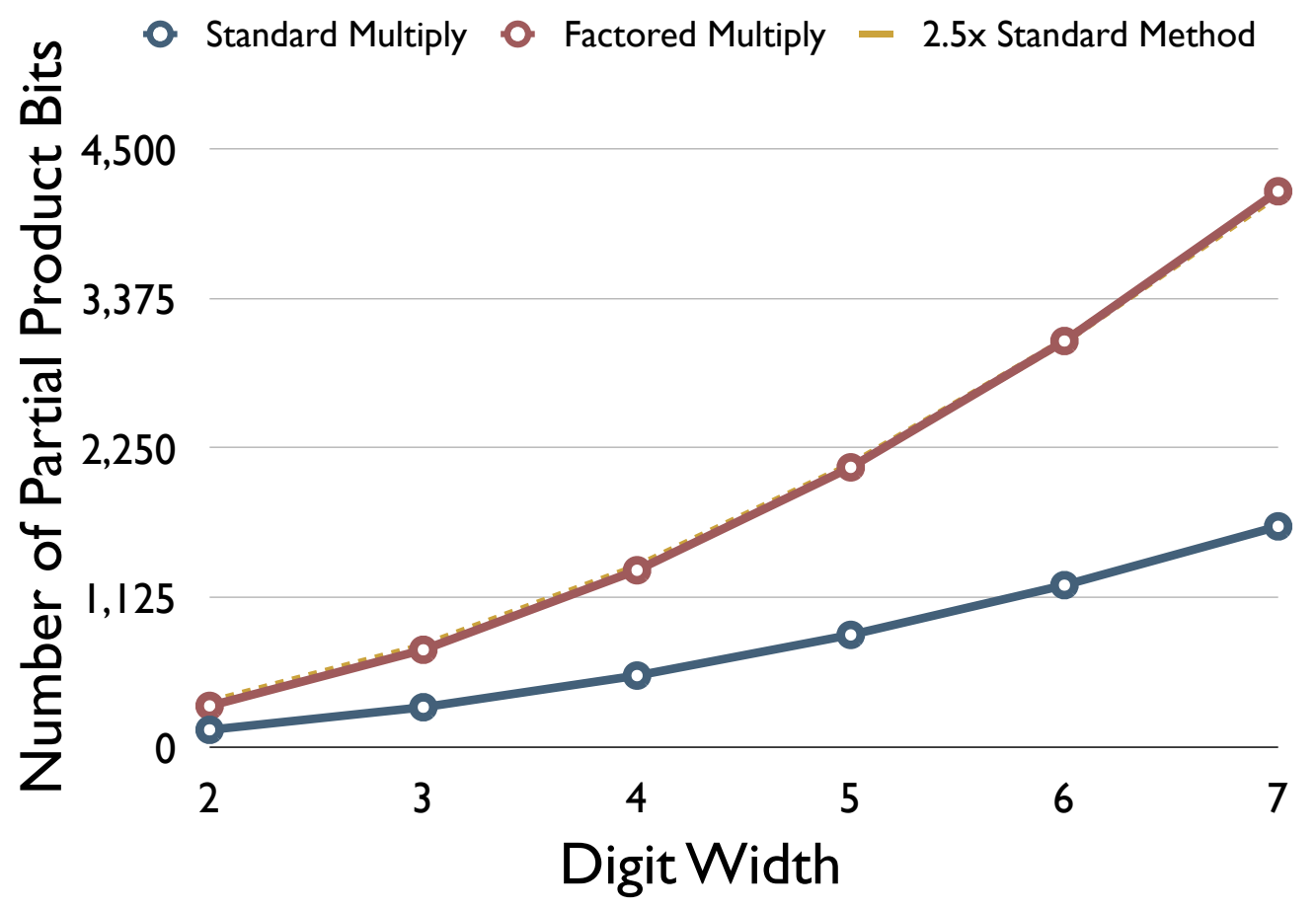

Figure A.3: Number of Partial Product Bits

For the factored algorithm, there are

$$
\begin{gathered}
2 \nu n+2 \nu\left(n^{2}-n\right)+(2 \nu+2) \frac{n^{2}-n}{2}= \\
(3 \nu+1) n^{2}-(\nu+1) n
\end{gathered}
$$

bits of partial product to be added. Additionally, there are $2 \frac{n^{2}-n}{2}$ subtractions to be performed before the multiplications, each with 2 operands of width $\nu+1$ bits, for a total of $2(\nu+1)\left(n^{2}-n\right)$ input bits to the subtractors. As a rough measure of adder area, we lump these subtraction bits in with the partial product adder tree bits, and obtain

$$
(5 \nu+3) n^{2}-(\nu+1) n
$$

Asymptotically, this amounts to a factor of 2.5 increase in the adder network complexity over the conventional method. Figure A.3 shows this effect graphically. 
$\square$ I7xI7 $\rightarrow 34$ bit unsigned multiply

18 bit subtract, $18 \times 18 \rightarrow 36$ bit signed multiply

Reused partial product, no multiply

Figure A.4: Legend for Partial Product Arrays

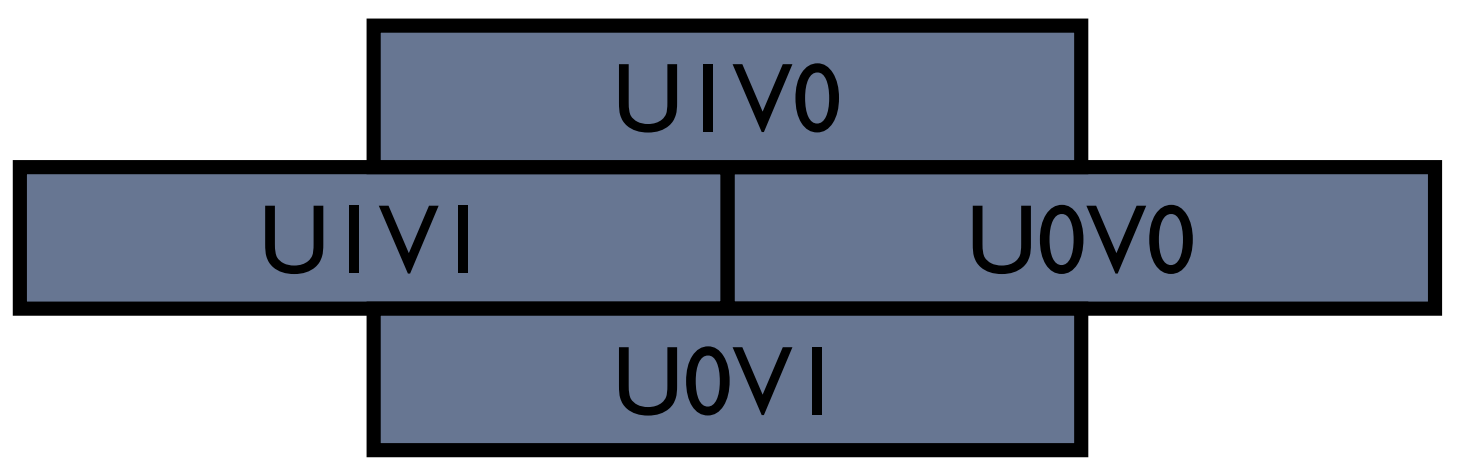

Figure A.5: Standard Partial Product Array for Single Precision Multiply

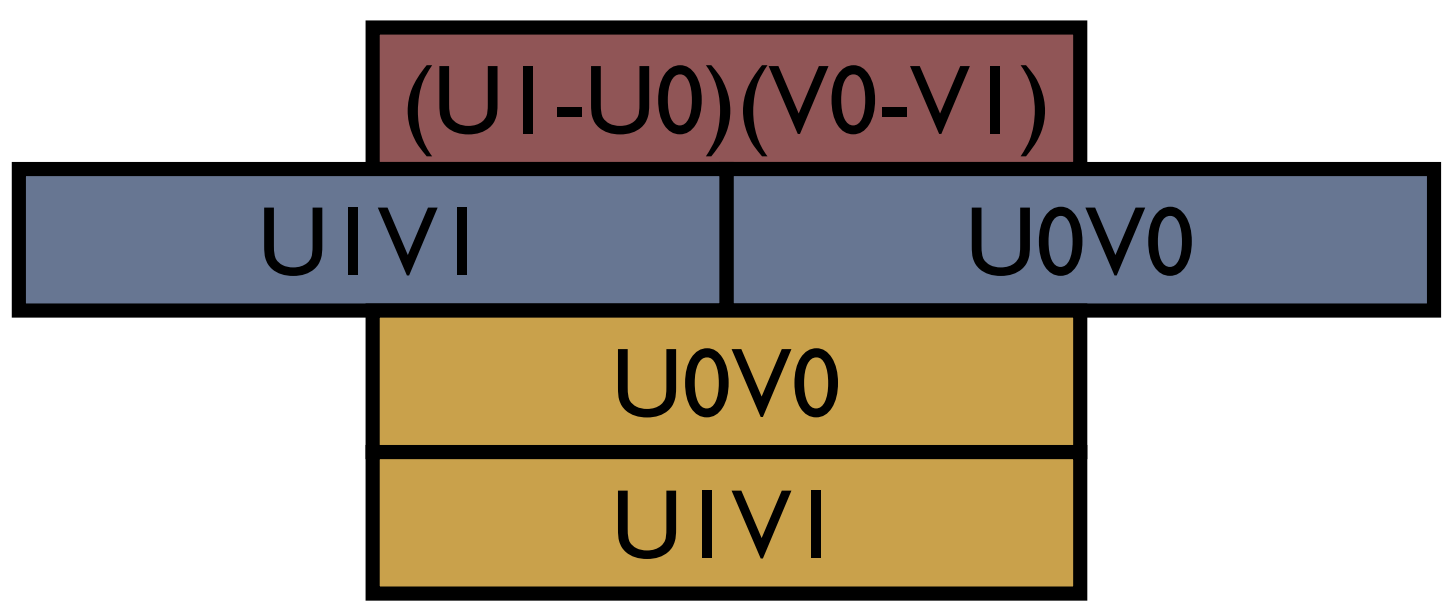

Figure A.6: Factored Partial Product Array for Single Precision Multiply 


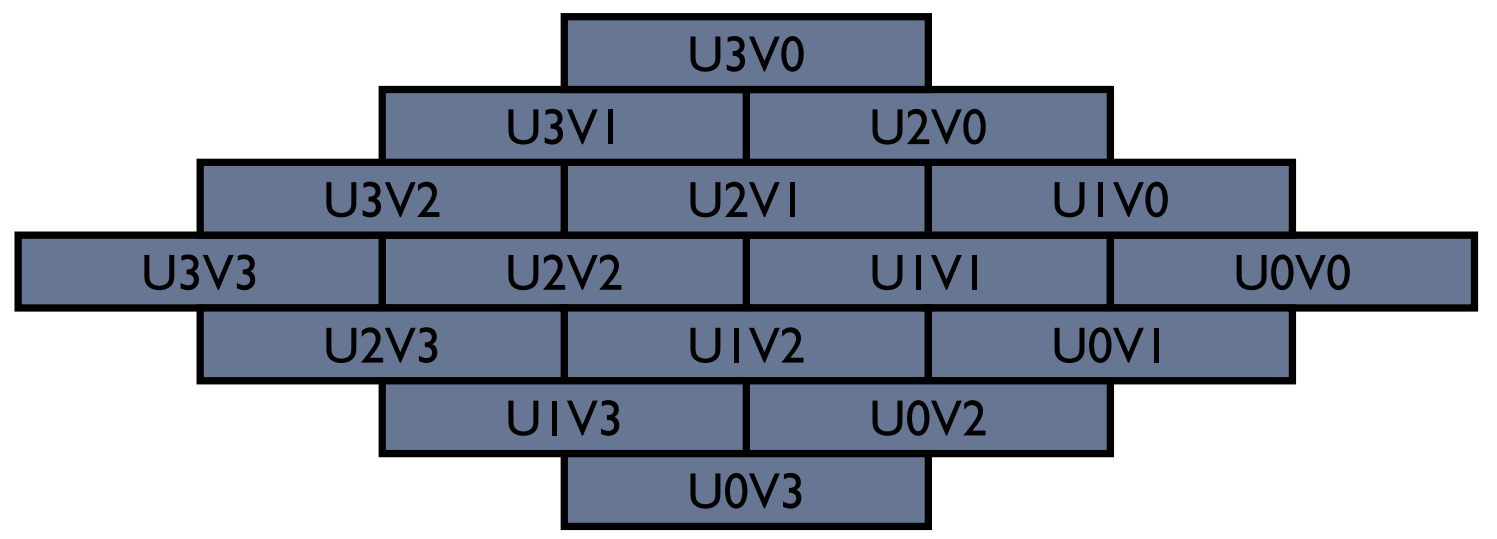

Figure A.7: Standard Partial Product Array for Double Precision Multiply

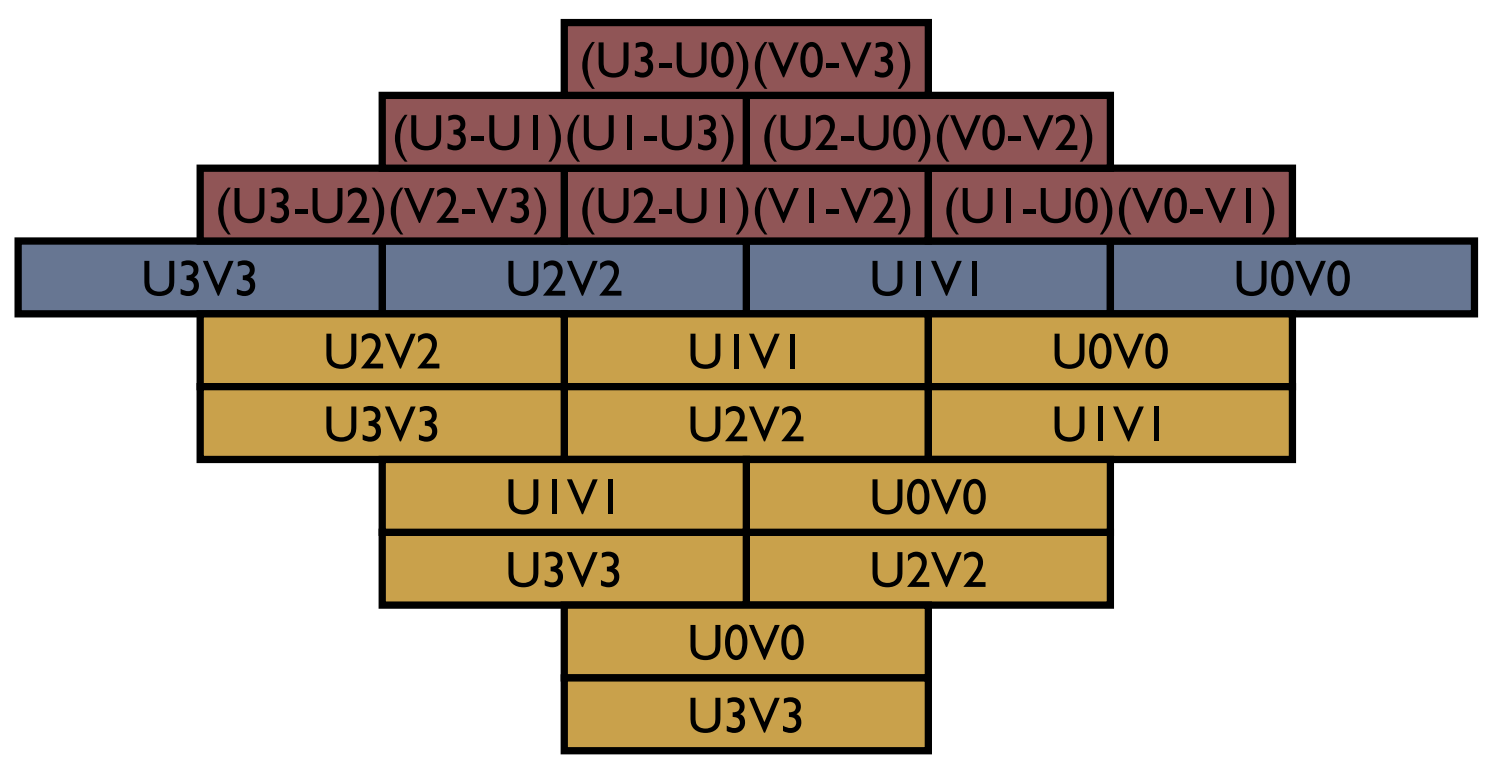

Figure A.8: Factored Partial Product Array for Double Precision Multiply 


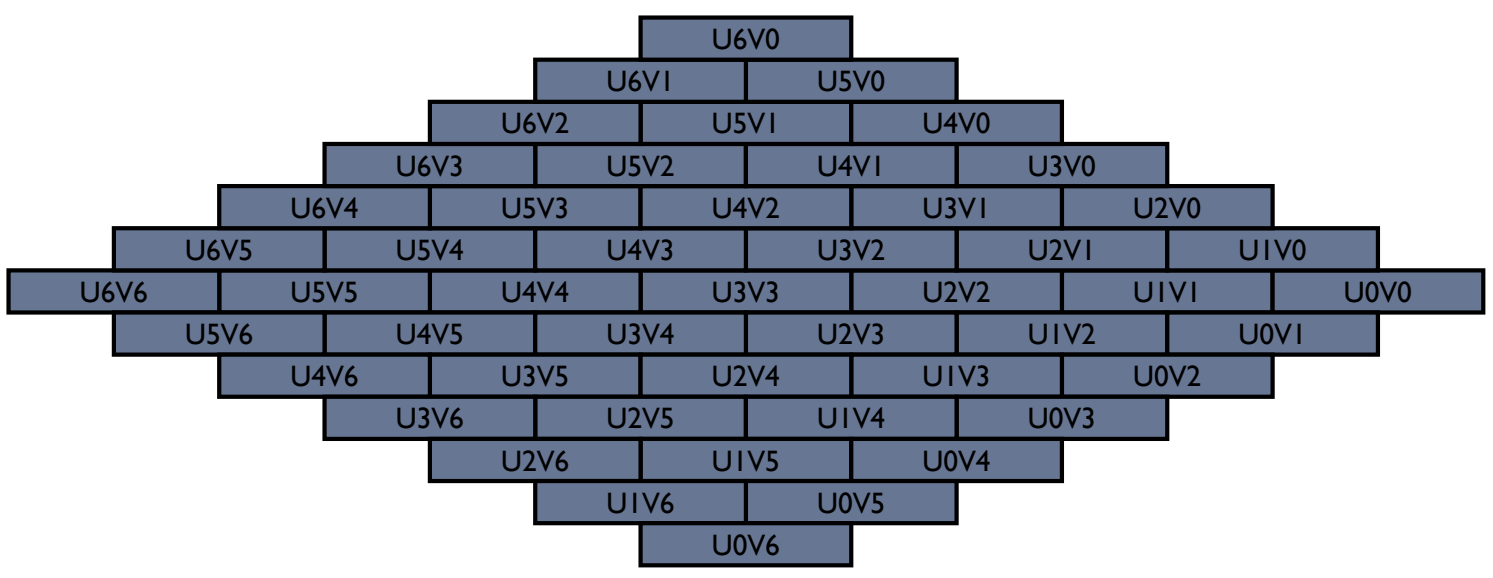

Figure A.9: Standard Partial Product Array for Quadruple Precision Multiply

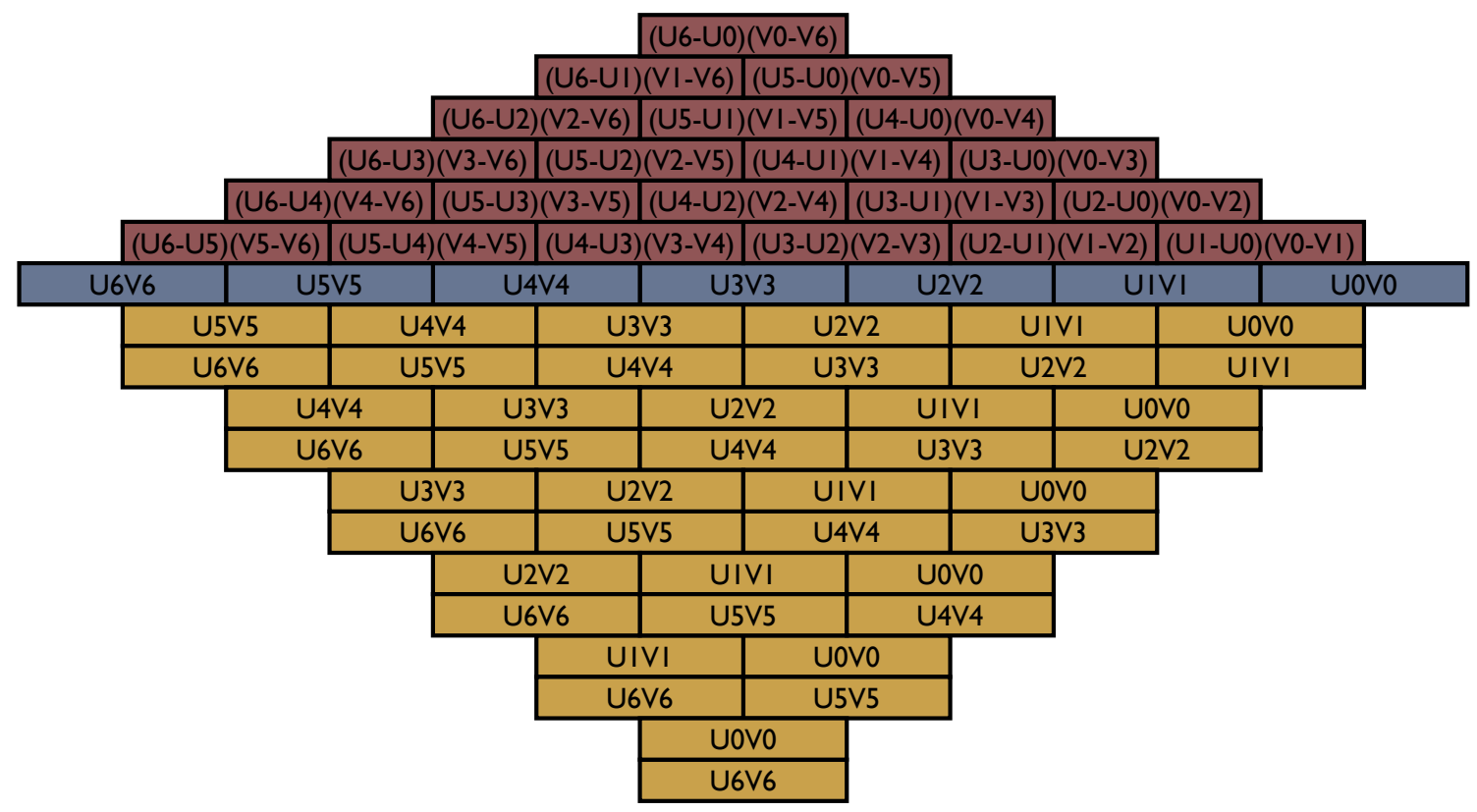

Figure A.10: Factored Partial Product Array for Quadruple Precision Multiply 
Figures A.5, A.6, A.7, A.8, A.9, and A.10 show partial product arrays for different multiplications, with and without the factorization applied. The horizontal position of each partial product is determined by its arithmetic weight, the vertical position does not carry any meaning. The product is computed by summing all the partial products vertically.

\section{A.4 Implementation}

An unpipelined multiplier which utilizes this factorization for an arbitrary operand width was implemented in JHDL. The implementation was more of a proof of concept to show that the correct results could be obtained in digital hardware, and its adder network did sign extension in a clumsy way which had a large area penalty. More optimization of the adder structure is possible, which would lessen the LUT count penalty seen with the factored multiplier presented here.

Table A.1: Multiplier Sizes

\begin{tabular}{|l|l|l|}
\hline Multiplier Type & Slices & Embedded Multipliers \\
\hline \hline Block Multiplier 18 bit signed & 0 & 1 \\
\hline LUT Multiplier 18 bit signed & 208 & 0 \\
\hline \hline Standard Single Precision 24 bit unsigned & 40 & 4 \\
\hline Reduced Single Precision 24 bit unsigned & 248 & 3 \\
\hline Factored Single Precision 24 bit unsigned & 68 & 3 \\
\hline \hline Standard Double Precision 53 bit unsigned & 227 & 16 \\
\hline Reduced Double Precision 53 bit unsigned & 1475 & 10 \\
\hline Factored Double Precision 53 bit unsigned & 566 & 10 \\
\hline \hline Standard Quadruple Precision 113 bit unsigned & 810 & 49 \\
\hline Reduced Quadruple Precision 113 bit unsigned & 5178 & 28 \\
\hline Factored Quadruple Precision 113 bit unsigned & 2245 & 28 \\
\hline
\end{tabular}

Table A.1 shows mapped results from a variety of multipliers. The first group shows that an 18 bit signed multiplier, equivalent to the embedded multiplier, costs 208 slices. The "Standard" multipliers referred to are normal multipliers, stitched 


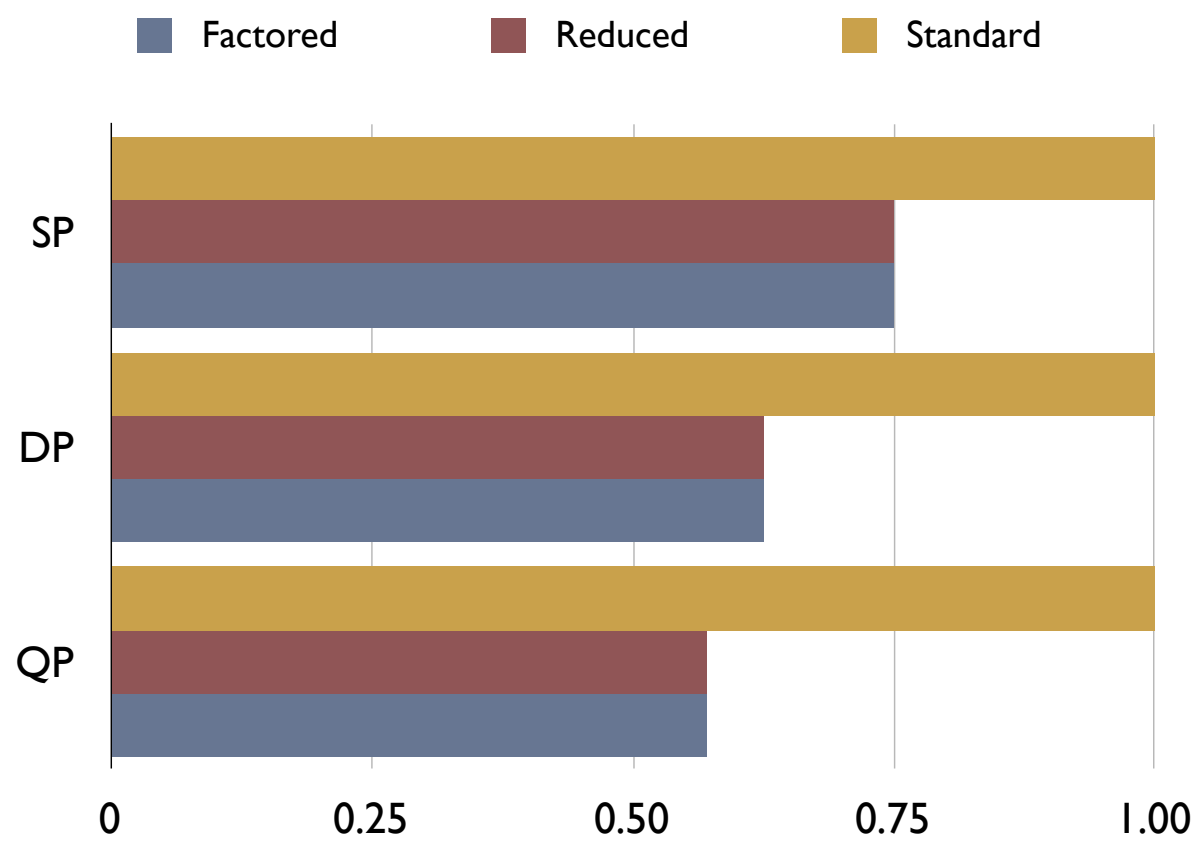

Figure A.11: Normalized Embedded Multiplier Usage

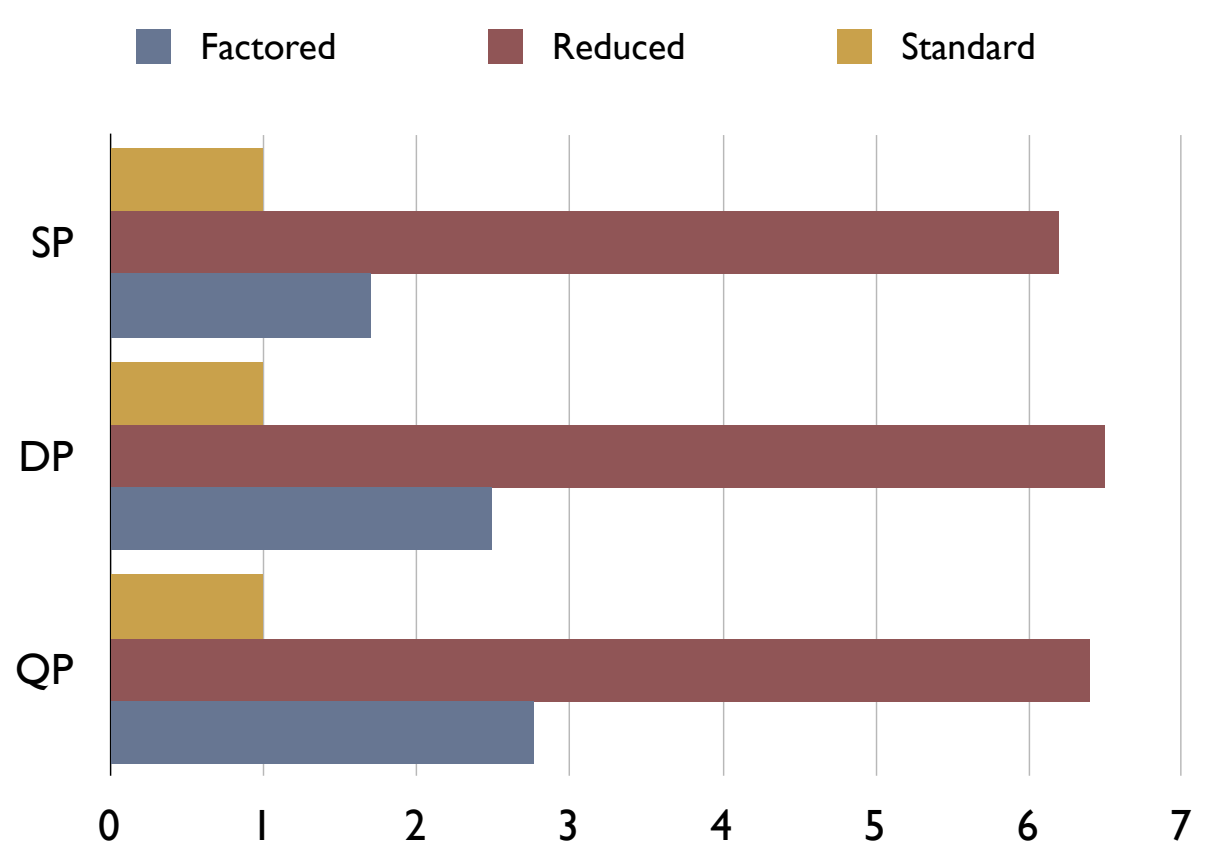

Figure A.12: Normalized Multiplier Slice Usage 
together from $n^{2}$ embedded multipliers. The "Reduced" multipliers illustrate the area penalty when using the standard multiplication algorithm, but reducing the embedded multiplier count by using $n+\frac{n^{2}-n}{2}$ embedded multipliers, and implementing the other $n^{2}-\left(n+\frac{n^{2}-n}{2}\right) 18 \times 18$ bit multipliers using LUT multipliers. The "Reduced" multipliers have slice counts extrapolated from the "Standard" multipliers with some of the embedded multipliers replaced by LUT multipliers, no actual circuits were built to obtain their sizes. The "Factored" multipliers use the factorization presented in this chapter.

Figure A.11 shows embedded multiplier usage, normalized to the standard multiplication algorithm. Figure A.12 shows how many slices are used for the multiplication.

The key point is that the factored multipliers have approximately a $2-4 \mathrm{x}$ slice count reduction over the reduced multipliers. This is important in multiplier limited scenarios, when a designer might be forced to implement some of the multiplication in LUTs instead of embedded multipliers. This factorization is therefore useful for embedded multiplier constrained circuits, such as those which might arise when implementing Quadruple Precision multiplication on FPGAs. 


\section{Bibliography}

[1] K. Underwood, "FPGAs vs. CPUs: Trends in Peak Floating-Point Performance," ACM/SIGDA Twelfth ACM International Symposium on FieldProgrammable Gate Arrays (FPGA'04), 2004.

[2] P. E. Cerruzi, A History of Modern Computing, 2nd ed. Cambridge, Massachussets: MIT Press, 2003.

[3] G. E. Moore, "Cramming More Components Onto Integrated Circuits," Electronics, vol. 38, no. 8, 1965.

[4] IEEE Standard for Binary Floating-Point Arithmetic, ANSI/IEEE Std 7541985 ed., IEEE Standards Board, 1985.

[5] IEEE Standard for Radix-Independent Floating-Point Arithmetic, ANSI/IEEE Std 854-1987 ed., IEEE Standards Board, 1987.

[6] A. A. Gaffar, O. Mencer, W. Luk, P. Y. Cheung, and N. Shirazi, "Floating Point Bitwidth Analysis via Automatic Differentiation," Proceedings of the International Conference on Field Programmable Technology, 2002.

[7] J. Dido et al., "A Flexible Floating-Point Format for Optimizing Data-Paths and Operators in FPGA Based DSPs," ACM/SIGDA Tenth ACM International Symposium on Field-Programmable Gate Arrays (FPGA'02), 2002.

[8] R. K. Yu and G. B. Zyner, "167 MHz Radix-4 Floating Point Multiplier," Proceedings of the IEEE Symposium on Computer Arithmetic, 1995. 
[9] X. Wang and B. Nelson, "Tradeoffs of Designing Floating-Point Division and Square Root on Virtex FPGAs," Proceedings of the IEEE Symposium on FPGAs for Custom Computing Machines (FCCM'03), pp. 195-203, 2003.

[10] D. W. Matula, "Base Conversion Mappings," Proceedings of the American Federation of Information Processing Societies, vol. 30, pp. 311-318, 1967.

[11] J. Liang, R.Tessier, and O. Mencer, "Floating Point Unit Generation and Evalution for FPGAs," Proceedings of the IEEE Symposium on Field-Programmable Custom Computing Machines (FCCM'03), pp. 185-194, 2003.

[12] DRAFT Standard for Floating-Point Arithmetic, P754/d0.10.2-2005 february 24 15:20 ed., Institute of Electrical and Electronics Engineers, Inc., http://754r.ucbtest.org/, 2005.

[13] B. Parhami, Computer Arithmetic: Algorithms and Hardware Designs. New York: Oxford University Press, 2000.

[14] D. M. Priest, "Fast Table-Driven Algorithms for Interval Elementary Functions," Proceedings of the 13th IEEE Symposium on Computer Arithmetic, pp. 168-174, 1997.

[15] D. E. Knuth, The Art of Computer Programming, 3rd ed. Reading, Massachussets: Addison Wesley, 1998, vol. 2.

[16] D. H. Bailey, "High-Precision Arithmetic in Scientific Computation," Computing in Science and Engineering, 2005.

[17] A. Akkaş and M. J. Schulte, "A Quadruple Precision and Dual Double Precision Floating-Point Multiplier," Proceedings of the Euromicro Symposium on Digital System Design (DSD'03), 2003.

[18] R. P. Brent, "On the Precision Attainable with Various Floating-Point Number Systems," IEEE Transactions on Computers, vol. C-22, pp. 601-607, June 1973. 
[19] G. Gerwig et al., "The IBM eServer z990 Floating-Point Unit," IBM Journal of Research and Development, vol. 48, no. 3/4, 2004.

[20] E. M. Schwarz, R. M. Smith, and C. A. Krygowski, "The S/390 G5 Floating Point Unit Supporting Hex and Binary Architecture," Proceedings of the 14th IEEE Symposium on Computer Arithmetic, 1999.

[21] M. F. Cowlishaw, "Decimal Floating-Point: Algorism for Computers," Proceedings of the 16th IEEE Symposium on Computer Arithmetic (ARITH'03), 2003.

[22] W. S. Brown and P. L. Richman, "The Choice of Base," Communications of the $A C M$, vol. 12, no. 10, pp. 560-561, October 1969.

[23] iAPX 86, 88, 186 and 188 User's Manual: Programmer's Reference, Intel Corporation, Santa Clara, California, 1985.

[24] E. Hokenek, R. K. Montoye, and P. W. Cook, "Second-Generation RISC Floating Point with Multiply-Add Fused," IEEE Journal of Solid-State Circuits, vol. 25, no. 5, pp. 1207-1213, 1990.

[25] Y. Voronenko and M. Puschel, "Automatic generation of implementations for DSP transforms on fused multiply-add architectures," Proceedings of the International Conference on Acoustics, Speech, and Signal Processing (ICASSP'04), vol. 5, pp. 101-104, 2004.

[26] B. Fagin and C. Renard, "Field Programmable Gate Arrays and Floating Point Arithmetic," IEEE Transactions on VLSI, vol. 2, no. 3, pp. 365-367, 1994.

[27] N. Shirazi, A. Walters, and P. Athanas, "Quantitative Analysis of Floating Point Arithmetic on FPGA Based Custom Computing Machines," Proceedings of the IEEE Symposium on FPGAs for Custom Computing Machines (FCCM'95), pp. 155-162, 1995.

[28] L. Louca, T. A. Cook, and W. H. Johnson, "Implementation of IEEE Single Precision Floating Point Addition and Multiplication on FPGAs," Proceedings of 
the IEEE Symposium on FPGAs for Custom Computing Machines (FCCM'96), pp. 107-116, 1996.

[29] "Xilinx, Incorporated," http://www.xilinx.com.

[30] "Altera Corporation," http://www.altera.com.

[31] "Lattice Semiconductor Corporation," http://www.latticesemi.com.

[32] E. Roesler and B. Nelson, "Novel Optimizations for Hardware Floating-Point Units in a Modern FPGA Architecture," Proceedings of the 12th International Workshop on Field Programmable Logic and Applications (FPL'02), pp. 637646, 2002.

[33] B. Lee and N. Burgess, "Parameterisable Floating-point Operations on FPGA," Conference Record of the Thirty-Sixth Asilomar Conference on Signals, Systems, and Computers, 2002.

[34] G. Govindu et al., "Analysis of High-performance Floating-point Arithmetic on FPGAs," Proceedings of the 18th International Parallel and Distributed Processing Symposium, 2004.

[35] P. Belanovic and M. Leeser, "A Library of Parameterized Floating Point Modules and Their Use." Proceedings of the 12th International Workshop on Field Programmable Logic and Applications (FPL'02), 2002.

[36] W. D. Smith and A. R. Schnore, "Towards an RCC-based Accelerator for Computational Fluid Dynamics Applications," Proceedings of the International Conference on Engineering of Reconfigurable Systems and Algorithms (ERSA'03), pp. 222-231, 2003.

[37] M. Gokhale et al., "Monte Carlo Radiative Heat Transfer Simulation on a Reconfiguable Computer," Proceedings of the 14th International Workshop on Field Programmable Logic and Applications (FPL'04), 2004. 
[38] H. A. H. Fahmy and M. J. Flynn, "The case for a redundant format in floating point arithmetic," Proceedings of the 16th IEEE Symposium on Computer Arithmetic, 2003.

[39] F. Fang, T. Chen, and R. A. Rutenbar, "Lightweight Floating-Point Arithmetic: Case Study of Inverse Discrete Cosine Transform," EURASIP Journal of Applied Signal Processing, pp. 879-892, September 2002.

[40] J. Detrey and F. de Dinechin, "FPLibrary, a VHDL Library of Parameterisable Floating-Point and LNS Operators for FPGA," http://perso.enslyon.fr/jeremie.detrey/FPLibrary/, 2004.

[41] M. D. Ercegovac and T. Lang, Digital Arithmetic. San Francisco: Morgan Kaufmann, 2004.

[42] B. Hutchings et al., "A CAD Suite for High-Performance FPGA Design," Proceedings of the IEEE Symposium on Field-Programmable Custom Computing Machines (FCCM'99), pp. 12-24, 1999.

[43] D. W. Matula, "Towards an abstract mathematical theory of floating-point arithmetic," Proceedings of the American Federation of Information Processing Societies, vol. 34, pp. 765-772, 1969.

[44] — - "In-and-out conversions," Communications of the ACM, vol. 11, no. 1, pp. $47-50,1968$.

[45] —_, "The base conversion theorem," Proceedings of the American Mathematical Society, vol. 19, no. 3, pp. 716-723, 1968.

[46] — - "A Formalization of Floating-Point Numeric Base Conversion," IEEE Transactions on Computers, vol. C-19, pp. 681-692, August 1970.

[47] H. Kuki and W. Cody, "A Statistical Study of the Accuracy of Floating Point Number Systems," Communications of the ACM, vol. 16, no. 4, pp. 223-230, 1973. 
[48] J. H. Wilkerson, Rounding Errors in Algebraic Processes. Englewood Cliffs, N.J.: Prentice-Hall, 1963.

[49] C. T. Ewe, P. Y. K. Cheung, and G. A. Constantinides, "Dual Fixed-Point: An Efficient Alternative to Floating-Point Computation," Proceedings of the 14 th International Workshop on Field Programmable Logic and Applications (FPL'04), 2004.

[50] W. B. Ligon, S. P. McMillan, G. Monn, F. Stivers, K. Schoonover, and K. D. Underwood, "A Re-evaluation of the Practicality of Floating-Point on FPGAs," Proceedings of the IEEE Symposium on FPGAs for Custom Computing Machines (FCCM'98), pp. 206-215, 1998.

[51] Z. Luo and M. Martonosi, "Accelerating Pipelined Integer and Floating-Point Accumulations in Configurable Hardware with Delayed Addition Techniques," IEEE Transactions on Computers, vol. 49, no. 3, pp. 208-218, 2000.

[52] Y. Dou, S. Vassiliadis, G. K. Kuzmanov, and G. N. Gaydadjiev, "64-bit FloatingPoint FPGA Matrix Multiplication," ACM/SIGDA Thirteenth ACM International Symposium on Field-Programmable Gate Arrays (FPGA'05), 2005. 